\title{
Discretization and Solution of Elliptic PDEs-A Digital Signal Processing Approach
}

\author{
C.-C. JAY KUO, MEMBER, IEEE, AND BERNARD C. LEVY, SENIOR MEMBER, IEEE
}

A digital signal processing (DSP) approach is used to study numerical methods for discretizing and solving linear elliptic partial differential equations (PDEs). Whereas conventional PDE anal$y$ sis techniques rely on matrix analysis and on a space-domain point of view to study the performance of solution methods, the DSP approach described here relies on frequency domain analysis and on multidimensional DSP techniques. This tutorial paper dis cusses both discretization schemes and solution methods. In the area of discretization, mode-dependent finite-difference schemes for general second-order elliptic PDEs are examined, and are illustrated by considering the Poisson, Helmholtz, and convection-diffusion equations as examples. In the area of solution methods, we focus on methods applicable to self-adjoint positive definite elliptic PDEs. Both direct and iterative methods are discussed, which include fast Poisson solvers, elementary and accelerated relaxation methods, multigrid methods, preconditioned conjugate gradient methods and domain decomposition techniques. In addition to describing these methods in a DSP setting, an up-to-date survey of recent developments is also provided.

\section{INTRODUCTION}

Many physical and engineering systems are described by partial differential equations (PDEs). It is generally impossible to obtain closed-form analytical solutions for these equations due to the irregularity of problem domains, and because coefficients are usually spatially varying. Consequently, the numerical solution of PDEs plays an important role in understanding and simulating a wide variety of physical phenomena. Since the late 1940s, the gradual emergence of high-speed computers, culminating with the introduction of supercomputers, has made it possible for researchers to test and develop new PDE solution techniques. The amount of research activity concerned with the numerical analysis of PDEs has therefore been growing very rapidly. Many discretization schemes, computational algo-

Manuscript received December 4, 1989; revised August 22, 1990 The first author was supported by the USC Faculty Research and Innovation Fund and by a National Science Foundation Research Initiation Award. The second author was supported by the National Science Foundation under Grant ECS-8700903.

C.-C. J. Kuo is with the Department of Electrical Engineering Systems, University of Southern California (USC), University Park, Los Angeles, CA 90089-0272, USA.

B. C. Levy is with the Department of Electrical Engineering and Computer Science, University of California-Davis (UC-Davis), Davis, CA 95616, USA.

IEEE Log Number 9040105. rithms, and novel computer architectures have been proposed to solve PDEs efficiently. In spite of these developments, the numerical solution of PDEs is still one of the most challenging areas of numerical analysis due to the versatile and often complicated structure of PDEs, and because of the large amount of variables that need to be computed for two or higher dimensional problems.

In this survey, we focus our attention on the discretization and solution of 2-D second-order linear elliptic PDEs of the form

$$
a \frac{\partial^{2} u}{\partial x^{2}}+b \frac{\partial^{2} u}{\partial y^{2}}+c \frac{\partial u}{\partial x}+d \frac{\partial u}{\partial y}+e u=f
$$

with $a b>0$, where the coefficients are in general functions of $x$ and $y$. Elliptic PDEs are often used to characterize the steady-state behavior of physical systems defined over a bounded domain. In this context, boundary conditions representing experimental conditions are usually imposed on the domain boundary, thus yielding a boundary-value problem. The familiar Laplace, Poisson, Helmholtz and convection-diffusion equations are all special cases of (1.1). The solution of (1.1) has therefore a wide range of applications [13], [88].

Elliptic PDEs can be divided into self-adjoint positive definite, indefinite and nonself-adjoint equations, depending on the eigenvalues of the associated differential operator. If an operator is self-adjoint, it has a real spectrum (eigenvalues). Furthermore, if it is positive definite, all its eigenvalues are positive. The discretization of self-adjoint positive definite differential operators leads to symmetric positive definite (SPD) matrices. In contrast, the discretization of nonself-adjoint elliptic operators gives rise to nonsymmetric matrices whose eigenvalues are in general complex. It is customary to use the Poisson, Helmholtz, and convection-diffusion equations on the unit square $\Omega=[0$, $1]^{2}$ with appropriate boundary conditions as model problems for self-adjoint positive definite, indefinite and nonself-adjoint elliptic PDEs, respectively. They can be expressed as follows.

Poisson equation:

$$
\frac{\partial^{2} u}{\partial x^{2}}+\frac{\partial^{2} u}{\partial y^{2}}=f
$$

0018-9219/90/1200-1808\$01.00 우 1990 IEEE 
Helmholtz equation:

$$
\frac{\partial^{2} u}{\partial x^{2}}+\frac{\partial^{2} u}{\partial y^{2}}+\omega^{2} u=f
$$

Convection-diffusion equation:

$$
\frac{\partial^{2} u}{\partial x^{2}}+\frac{\partial^{2} u}{\partial y^{2}}+c \frac{\partial u}{\partial x}+d \frac{\partial u}{\partial y}=f .
$$

Generally speaking, the numerical solution of PDEs involves two tasks: (a) choosing a discretization scheme to transform the PDE of interest into a discrete problem that approximates it, and (b) selecting a solution method for the discretized problem. These two tasks are usually performed separately for single grid solution techniques, but they are combined for multigrid methods. For expository purposes, since the goals of the discretization and solution steps are different, they will be examined independently. In this paper, we study the discretization of all three model problems (1.2)-(1.4). As to solution methods, the design and analysis of iterative algorithms for solving self-adjoint positive definite elliptic PDEs has reached an advanced state of development, whereas a complete theory is not yet available for indefinite and nonself-adjoint PDEs. Thus, we focus on the solution of self-adjoint positive definite PDEs modeled by the Poisson equation (1.2).

Our exposition relies on a Digital Signal Processing (DSP) approach [31], [34], [78], [85]. From the DSP viewpoint, 2-D differential and finite-difference operators correspond to 2-D analog and digital filters, respectively. The discretization of PDEs specifies an approximation problem, i.e., how to match the spectra of analog and digital filters. The solution of PDEs requires the implementation of a deconvolution filter which recovers the input $u$ from the output $f$ given by (1.1). Thus, the discretization and solution of PDEs can be formulated as multidimensional filter specification and filter design problems, respectively.

A key step in deriving discretization schemes is the selection of a set of test functions for which the discretized operator must behave in the same way as the original differential operator. It turns out that a good set of test functions can be chosen by using concepts of linear systems theory. Roughly speaking, they are obtained by examining the zeros of the system function corresponding to the differential operator. This approach leads to the mode-dependent discretization scheme described in Section II.

The discretization procedure leads to a system of finitedifference equations, which are often solved iteratively. The convergence rate of iterative methods is traditionally studied within the framework of matrix iterative analysis [14], [96], [102]. This form of analysis uses tools from numerical linear algebra, where special concepts such as those of $L-, M-$, and consistently ordered matrices and related inequalities are introduced to facilitate the characterization of the convergence property. The advantage of matrix anal$y$ sis is its general applicability. It can be applied to PDEs with irregular geometries and spatially varying coefficients, or which are discretized with nonuniform grids, as long as the corresponding iteration matrices satisfy the desired properties.

An approach complementing the matrix formulation relies on model problem analysis, whereby the convergence rate of a given iterative method is analyzed for a sim- ple model problem. This form of analysis has several advantages. First, it is much simpler and therefore provides some insight into the behavior of the algorithms that we study. Secondly, the estimates that are provided by this approach for parameters such as the optimum relaxation parameter for the Successive Over-Relaxation (SOR) method, or the smoothing rate of multigrid methods, are usually much sharper than comparable estimates provided by matrix analysis. Finally, the actual convergence behavior of an iterative method for a general class of problems can be well predicted by the model problem approach, as long as the model problem is chosen appropriately.

The model problem approach relies heavily on Fourier analysis. In this survey, we show that it is in fact closely related to the digital filtering concept appearing in multidimensional DSP. Note that this relation was also pointed out in the earlier work of [15] and [90], but only briefly. Several examples are given below. Accelerated relaxation methods such as the SOR and Chebyshev iterative methods can be viewed as parametrized lowpass filters for the error between the initial guess and the true solution, where the parameters are chosen to optimize the filtering characteristics. The incomplete $\mathrm{LU}$ preconditioning technique for the conjugate gradient method can be interpreted as corresponding to the approximation of a 2-D noncausal FIR filter by the product of two causal and anticausal 2-D FIR filters. The difficulty in that respect lies in the fact that since 2-D polynomials are generally not factorable, the 2-D causal and anticausal filters obtained by spectral factorization have infinite support, and need therefore to be approximated. Finally, if we consider multigrid solution methods, the interpolation and restriction operators appearing in the description of these algorithms are special cases of sampling-rate conversion operations occurring in multirate signal processing. The details of all the above examples will be discussed below. The main purpose of these examples is to illustrate the fact that many tools and concepts arising in the solution of elliptic PDEs are amenable to interpretation and analysis from the point of view of multidimensional DSP.

This survey contains two parts: the first part (Section II) considers discretization schemes, whereas the second part (Sections III-IX) examines solution methods. Readers seeking to locate quickly topics of interest may want to consult the following table of contents.

II. Mode-Dependent Discretization

A. The Mode-Dependent Finite-Difference Discretization Approach

B. Discretization of Homogeneous Boundary-Value ODEs

C. Discretization of Homogeneous Boundary-Value PDEs

D. Historical Notes

III. Solution of Self-Adjoint Positive Definite Elliptic PDEs: Problem Formulation

A. The Model Poisson Problem

B. Orderings

C. Fourier Analysis

D. Summary

IV. Direct Methods

A. FFT Solvers

B. Other Direct Methods 
V. Relaxation Methods and Their Acceleration
A. Elementary Relaxation Methods
B. SOR Acceleration
C. Polynomial Acceleration
D. Historical Notes

VI. Multigrid Methods

A. Two-grid Iteration

B. Solution of the 1-D Poisson Problem

C. Solution of the 2-D Poisson Problem

D. Historical Notes

VII. Preconditioned Conjugate Gradient Methods

A. The Preconditioned Conjugate Gradient (PCG) Algorithm

B. Preconditioners Based on Incomplete Factorization

C. Multilevel Preconditioners Based on Filtering

D. Historical Notes

VIII. Domain Decomposition Methods

A. Capacitance Matrix Formulation

B. Fourier Analysis of the Capacitance System

C. Preconditioners for the Capacitance Matrix

D. Historical Notes

IX. Parallel Computation

Finally, we discuss future extensions and present some concluding remarks in Section $X$.

\section{Mode-Dependent Discretization}

Three types of discretization techniques, the finite-difference, finite-element, and spectral methods, are commonly used to discretize spatial partial differential operators. In this section, we focus our attention on modedependent finite-difference discretization schemes (which constitute an extension of standard finite-difference methods), since they are particularly interesting from a digital filtering point of view. The reader is referred to [72] and the references therein for a discussion of the relation existing between spectral and mode-dependent discretization methods, and for a brief overview of mode-dependent finite-element methods.

The analysis and design of mode-dependent finite-difference discretization schemes can be formulated in a simple way in the frequency domain. The Laplace and Z-transforms are used to represent the constant-coefficient differential operator and its discrete approximation by polynomial expressions of the transform variables $s$ and $z$. Then, the selection of a mode-dependent discretization scheme becomes equivalent to requiring that the spectra of the continuous and discretized operators, and their derivatives, should match each other at a number of frequencies in the transform domain. In DSP terms, since we require that the spectra of the continuous and discretized operators should be as close as possible, the PDE discretization problem can therefore be viewed as a filter specification and design problem.

\section{A. The Mode-Dependent Finite-Difference Discretization Approach}

Consider a function of the form

$$
u(x)=\sum_{k=1}^{k}\left[c_{k 0}+c_{k 1} x+c_{k 2} \frac{x^{2}}{2 !}+\cdots+c_{k n_{k}} \frac{x^{n_{k}}}{\left(n_{k}\right) !}\right] e^{s_{k} x}
$$

where each term $x^{p} e^{s_{k} x}, 0 \leq p \leq n_{k}$, is called a mode of order $p$ at the frequency $s_{k}$. We are interested in approximating a linear $R$ th-order constant-coefficient differential operator operating on $u(x)$,

$$
L(D)=\sum_{r=0}^{R} a_{r} D^{r}
$$

where $D=d / d x$, by a $\left(r_{2}-r_{1}+1\right)$-point finite-difference operator

$$
L_{d}(E)=\sum_{r=r_{1}}^{r_{2}} b_{r} E^{r}
$$

where $E$ is the shift operator defined on an infinite uniform grid $\Omega_{h}$ with spacing $h$, i.e. for $n h,(n+r) h \in \Omega_{h}, E^{\prime} u(n h)=$ $u((n+r) h) . L_{d}$ corresponds to a forward, backward or central difference operator depending on whether $r_{1}=0, r_{2}=$ 0 or $-r_{1}=r_{2}$, respectively. We denote by

$$
P_{n}(s)=\left\{u(x): u(x)=e^{s x} \sum_{k=0}^{n} c_{k} x^{k}\right\}
$$

the space spanned by polynomials of degree at most $n$ multiplied by the factor $e^{s x}$. A mode-dependent finite-difference discretization scheme is obtained by selecting the coefficients $b_{r}$ of $L_{d}$ such that

$$
\begin{aligned}
& {\left[L_{d}(E)-L(D)\right] u(x)=0,} \\
& \text { for } u(x) \in C \text { and } x \in \Omega_{h}
\end{aligned}
$$

where $C$, which is called the coincident space of $L_{d}$, is the direct sum of subspaces of the form (2.3), i.e.

$$
C=\bigoplus_{k=1}^{K} P_{n_{k}}\left(s_{k}\right) \text {. }
$$

A mode in the coincident space $C$ is called a coincident mode, and its frequency is called a coincident frequency.

The above mode-dependent finite-difference scheme specification can be converted easily to the transform domain. Let $L(s)$ be the spectrum obtained by replacing $D$ with $s$ in (2.1) through the use of the Laplace transform, i.e.,

$$
L(s)=\sum_{r=0}^{R} a_{r} s^{r} .
$$

Let also $L_{d}(z)$ be the discrete spectrum obtained by using the $Z$-transform to replace $E$ by $z$ in (2.2), so that

$$
L_{d}(\mathrm{z})=\sum_{r=r_{1}}^{r_{1}} b_{r} z^{r}=\sum_{r=r_{r}}^{r_{2}} b_{r} \mathrm{e}^{r s h}
$$

where the last equality is due to the fact that since $E$ is related to $D$ via $E=e^{h D}[32]$, we have $z=e^{s h}$. Then, the difference $\Delta$ between $L$ and $L_{d}$ can be expressed in terms of the variable $s$ as

$$
\Delta(s)=L_{d}\left(e^{s h}\right)-L(s)
$$

and the mode-dependent finite-difference scheme specification (2.4)-(2.5) takes the form (see [72] for a proof)

$$
\Delta^{(p)}\left(s_{k}\right)=0 ; 0 \leq p \leq n_{k} ; 1 \leq k \leq K
$$

where

$$
\Delta^{(p)}\left(s_{k}\right)=\left.\frac{d^{p} \Delta(s)}{d s^{p}}\right|_{s=s_{k}} .
$$


It is usually easier to determine the coefficients $b_{r}$ of a modedependent finite-difference discretization scheme by using (2.7) rather than $(2.4)-(2.5)$.

The key element in the specification of a mode-dependent difference scheme is the choice of coincident space C. In the following two subsections, we discuss the selection of $C$ for several types of problems.

\section{B. Discretization of Homogeneous Boundary-Value ODEs}

Consider an $R$ th-order $(R=2 m)$ homogeneous differential equation

$$
L u=0, \quad \text { with } L=\sum_{r=0}^{2 m} a_{r} D^{r} \text { and } \quad a_{2 m}=1
$$

on the interval $[0,1]$, with given boundary conditions. We seek to discretize it with a $(2 m+1)$-point central difference scheme on a uniform grid with spacing $h$. The characteristic equation of $(2.8)$ is

$$
\begin{aligned}
L(s) & =s^{2 m}+a_{2 m-1} s^{2 m-1}+\cdots+a_{1} s+a_{0} \\
& =\Pi_{k=1}^{K}\left(s-s_{k}\right)^{n_{k}}=0
\end{aligned}
$$

with $\Sigma_{k=1}^{k} n_{k}=2 m$, where $s_{k}$ is a natural frequency of $L$ of order $n_{k}$. Then, the operator $L$ has the $2 m$-dimensional nullspace

$$
N_{L}=\bigoplus_{k=1}^{K} P_{n_{k}-1}\left(s_{k}\right)
$$

To determine uniquely a $(2 m+1)$-point finite difference scheme, we need to specify a $(2 m+1)$-dimensional coincident space $C$. However, since a homogeneous finite-difference equation can be scaled by an arbitrary constant, a $2 m$-dimensional coincident space $C$ is sufficient. An exact discretization for (2.8) is obtained by selecting

$$
C=N_{L} \text {. }
$$

For this choice, the relations (2.7) yield

$$
L_{d}(z)=A z^{-m} \prod_{k=1}^{k}\left(z-z_{k}\right)^{n_{k}}, \quad \text { with } z_{k}=e^{s_{k} h}
$$

where $A$ is a scaling factor and the multiplication factor $z^{-m}$ is due to the fact that we want $L_{d}(z)$ to be a central difference scheme. The choice of scaling factor $A$ does not affect the solution of the discretized equation

$$
L_{d}(E) u_{d}=0 .
$$

However, in order to analyze the discretization error $\Delta(s)$, it is convenient to choose $A$ such that $L_{d}\left(\mathrm{e}^{s h}\right)$ and $L(s)$ are consistent over fine grids. This constraint implies that $A$ must be proportional to $h^{-2 m}$, as $h$ goes to zero.

$1 D$ Laplace equation: For $L(D)=D^{2}$, we know that $N_{L}=$ $\{1, x\}$. The coincident modes have the same frequency $s_{k}$ $=0$. According to (2.11), we have

$$
L_{d}(E)=A E^{-1}(E-1)^{2}=A\left(E-2+E^{-1}\right) .
$$

If we choose $C=N_{L}+\left\{x^{2}\right\}$, the constant $A$ is uniquely determined. We obtain $A=h^{-2}$, and in this case (2.12) reduces to the standard 3-point central difference scheme for $D^{2}$.

$1 D$ convection-diffusion equation: Let $L(D)=D^{2}-a D$, with $a \neq 0$. Then, $N_{L}=\left\{1, \mathrm{e}^{a x}\right\}$ and $s_{k}=0$, a, so that in (2.11) we have

$$
\begin{aligned}
L_{d}(E) & =A E^{-1}(E-1)\left(E-e^{a h}\right) \\
& =A\left[E-\left(1+e^{a h}\right)+e^{a h} E^{-1}\right] .
\end{aligned}
$$

If we select $C=N_{L}+\{x\}$, we find that $A=a\left[h\left(e^{a h}-1\right)\right]^{-1}$, and (2.13) becomes identical to a scheme considered by Allen and Southwell [3].

\section{Discretization of Homogeneous Boundary-Value PDEs}

Consider a general 2D homogeneous boundary-value PDE on the square $[0,1]^{2}$

$$
L\left(D_{x}, D_{y}\right) u=0, \quad \text { with } L\left(D_{x}, D_{y}\right)=\sum_{r, s} a_{r, s} D_{x}^{r} D_{y}^{s}
$$

where

$$
D_{x}^{\prime}=\frac{\partial^{r}}{\partial x^{\prime \prime}}, \quad D_{y}^{s}=\frac{\partial^{s}}{\partial y^{s}}
$$

with appropriate boundary conditions. We discretize (2.14) with the finite-difference scheme

$$
L_{d}\left(E_{x}, E_{y}\right) u_{d}=0, \quad \text { where } L\left(E_{x}, E_{y}\right)=\sum_{r, s} b_{r, s} E_{x}^{r} E_{y}^{s}
$$

and where $E_{x}$ and $E_{y}$ denote respectively the shift operators in the $x$-and $y$-directions on the uniform grid $\Omega_{h_{x}, h_{y}}$ obtained by discretizing the unit square with horizontal and vertical meshes $h_{x}$ and $h_{y}$. Relying upon a natural generalization of the $1 \mathrm{D}$ case, we have the following correspondences between $2 \mathrm{D}$ space domain operators and transform domain variables

$$
D_{x} \leftrightarrow s_{x}, \quad D_{y} \leftrightarrow s_{y}, \quad E_{x} \leftrightarrow z_{x}, \quad E_{y} \leftrightarrow z_{y}
$$

where $s_{x}=\sigma_{x}+i \omega_{x}$ and $s_{y}=\sigma_{y}+i \omega_{y}$, and where the identities $E_{x}=e^{h_{x} D_{x}}, E_{y}=\mathrm{e}^{h_{y} D_{y}}, z_{x}=\mathrm{e}^{h_{x} s_{x}}$ and $z_{y}=\mathrm{e}^{h_{y} s_{y}}$ are satisfied. For simplicity, we now restrict our attention to the case where $h_{x}=h_{y}=h$.

Substituting $u=e^{s_{x} x+s_{y} y}$ inside (2.14), we obtain the characteristic equation

$$
\sum_{r, s} a_{t, s} s_{x}^{r} s_{y}^{s}=0 .
$$

Since the complex equation (2.17) imposes only two real constraints on the real and imaginary parts of the complex variables $s_{x}$ and $s_{y}$, there are infinitely many solutions to this equation and therefore infinitely many modes in $N_{L}$. It is not possible to approximate all modes in $N_{L}$ exactly. Thus, we have to select a finite-dimensional subspace $D_{L} \subset N_{L}$ called the dominant-mode space, as the coincident space $C$ for $L_{d}$. The determination of $D_{L}$ depends on a rough estimate of the local behavior of the solution. This information is usually provided by the structure of the PDE operator and of the boundary conditions. In this section, we restrict our attention to the case where the dominant modes are either oscillating or exponentially growing (decaying). In other words, coincident frequencies are selected among the sets

$$
\left\{\left(s_{x}, s_{y}\right):\left(s_{x}, s_{y}\right)=\left(\sigma_{x}, \sigma_{y}\right)\right\} \text { or }\left\{\left(s_{x}, s_{y}\right):\left(s_{x}, s_{y}\right)=\left(i \omega_{x}, i \omega_{y}\right)\right\} \text {. }
$$

Laplace equation: Let $L\left(D_{x}, D_{y}\right)=D_{x}^{2}+D_{y}^{2}$. Since only one frequency $\left(s_{x}, s_{y}\right)=(0,0)$ satisfies the characteristic equation and belongs to the sets $(2.18),(0,0)$ is selected as the unique coincident frequency. In this case, the mode-dependent and conventional discretization schemes are identical. 
The following 5-point, rotated 5-point and 9-point stencil discretization schemes have been derived by several approaches [32], [65], [80],

$$
\begin{aligned}
L_{d,+}\left(E_{x}, E_{y}\right)= & \frac{1}{h^{2}}\left(E_{x}+E_{x}^{-1}+E_{y}+E_{y}^{-1}-4\right) \\
L_{d, x}\left(E_{x}, E_{y}\right)= & \frac{1}{2 h^{2}}\left(E_{x} E_{y}+E_{x}^{-1} E_{y}+E_{x} E_{y}^{-1}+E_{x}^{-1} E_{y}^{-1}-4\right) \\
L_{d, 9}\left(E_{x}, E_{y}\right)= & \frac{1}{6 h^{2}}\left[4\left(E_{x}+E_{x}^{-1}+E_{y}+E_{y}^{-1}\right)\right. \\
& \left.+\left(E_{x} E_{y}+E_{x}^{-1} E_{y}+E_{x} E_{y}^{-1}+E_{x}^{-1} E_{y}^{-1}\right)-20\right]
\end{aligned}
$$

It is well known that the accuracy of the above schemes for discretizing the Laplace equation is $O\left(h^{2}\right), O\left(h^{2}\right)$ and $O\left(h^{6}\right)$, respectively.

We present now another derivation of these schemes by matching $L\left(s_{x}, s_{y}\right)$ and $L_{d}\left(z_{x}, z_{y}\right)$ at the coincident frequency $(0,0)$ in the transform domain. As before, we consider the expansion of $\Delta=L_{d}-L$ around $(0,0)$,

$$
\begin{aligned}
\Delta\left(s_{x}, s_{y}\right)= & \Delta^{(0,0)}(0,0)+\Delta^{(1,0)}(0,0) s_{x}+\Delta^{(0,7)}(0,0) s_{y} \\
& +\frac{1}{2}\left[\Delta^{(2,0)}(0,0) s_{x}^{2}+\Delta^{(1,1)}(0,0) 2 s_{x} s_{y}\right. \\
& \left.+\Delta^{(0,2)}(0,0) s_{y}^{2}\right] \\
& +\sum_{p+q \geq 3, p, q \geq 0} \Delta^{(p, q)}(0,0) \frac{1}{p ! q !} s_{x}^{p} s_{y}^{q}
\end{aligned}
$$

where

$$
\Delta^{(p, q)}(0,0)=\left.\frac{\partial^{p+q} \Delta\left(s_{x}, s_{y}\right)}{\partial^{p} s_{x} \partial^{q} s_{y}}\right|_{\left(s_{x}, s_{y}\right)=(0,0)}
$$

is a function of the grid size $h$. Hence, (2.22) is in fact a power series of $h$. Our derivation attempts to make the order of the residual terms in (2.22) as high as possible.

The discretization schemes (2.19) and (2.20) can be derived by requiring respectively that

$$
\begin{aligned}
\Delta^{(0,0)}(0,0) & =\Delta^{(1,0)}(0,0)=\Delta^{(0,1)}(0,0)=\Delta^{(2,0)}(0,0) \\
& =\Delta^{(0,2)}(0,0)=0
\end{aligned}
$$

and

$$
\begin{aligned}
\Delta^{(0,0)}(0,0) & =\Delta^{(1,0)}(0,0)=\Delta^{(0,1)}(0,0)=\Delta^{(1,1)}(0,0) \\
& =\Delta^{(2,0)}(0,0)=\Delta^{(0,2)}(0,0)=0 .
\end{aligned}
$$

Note the similarity between these requirements and (2.7). The above choice of constraints $\Delta^{(p, q)}(0,0)=0$ has taken into account the specific structure of operators $L_{d_{1}+}, L_{d_{1} \times}$ and $L$. For example, in the case of $L_{d, x}$, the symmetry properties of $L_{d, x}$ imply that $\Delta^{(2,0)}(0,0)=\Delta^{(0,2)}(0,0)$, so that among the six constraints which are used to specify $L_{d, x}\left(E_{x}, E_{y}\right)$, only five are independent.

By setting the coefficients of low order terms in (2.22) equal to zero, it is possible to obtain various high-order finite-difference discretization schemes. For example, to obtain the 9-point scheme (2.21), we need only to impose the requirement that this scheme should have an accuracy of $O\left(h^{6}\right)$ for modes satisfying the characteristic equation $s_{x}^{2}+s_{y}^{2}=0$. Then, substituting this equation inside (2.22) and setting coefficients up to order $h^{5}$ equal to zero, we obtain nine independent constraints which specify (2.21) uniquely.

Helmholtz equation: Let

$$
L\left(D_{x}, D_{y}\right)=D_{x}^{2}+D_{y}^{2}+\lambda^{2} .
$$

If $s_{x}$ and $s_{y}$ are purely imaginary, the characteristic equation becomes

$$
\omega_{x}^{2}+\omega_{y}^{2}=\lambda^{2},
$$

which is a circle in the $\omega_{x}-\omega_{y}$ plane, centered at the origin and with radius $|\lambda|$. There are infinitely many natural frequencies and, hence, there are many different ways to select coincident frequencies. Our choice is based on the following two considerations. First, if there is no further information about the dominant modes, a reasonable strategy consists in distributing the coincident frequencies uniformly along the contour (2.23). Second, we want to preserve the symmetry properties of $L$, so that the resulting discretization scheme will have a simple form and will be easy to implement.

Let us select

$\left(\omega_{x}, \omega_{y}\right)=\left(|\lambda| \cos \left(\frac{n}{2} \pi+\frac{1}{4} \pi\right), \quad|\lambda| \sin \left(\frac{n}{2} \pi+\frac{1}{4} \pi\right)\right)$,

$$
0 \leq n \leq 3
$$

as coincident frequencies as shown in Fig. 1(a). With this choice, the discretization can be performed independently

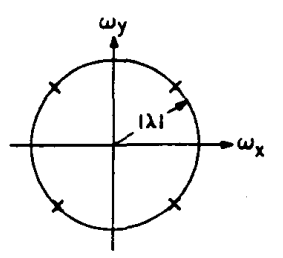

(a)

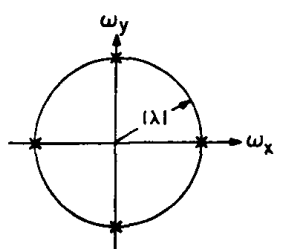

(b)

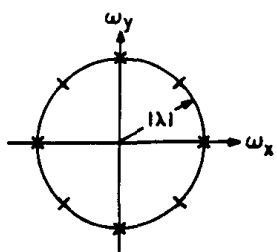

(c)

Fig. 1. Coincident frequencies of the mode-dependent (a) 5-point, (b) rotated 5-point, and (c) 9-point stencil discretization of the Helmholtz equation. 
in the $x$-and $y$-directions. The resulting scheme is

$$
\begin{aligned}
L_{d}\left(E_{x}, E_{y}\right)= & A\left[E_{x}^{-1}-2 \cos \left(\frac{|\lambda|}{\sqrt{2}} h\right)\right. \\
& \left.+E_{x}+\kappa\left(E_{y}^{-1}-2 \cos \left(\frac{|\lambda|}{\sqrt{2}} h\right)+E_{y}\right)\right] .
\end{aligned}
$$

Two parameters $A$ and $k$ remain undetermined. The parameter $k$ is selected such that the discretization error $\Delta\left(s_{x}, s_{y}\right)$ corresponding to natural frequencies is proportional to $O\left(h^{2}\right)$, and $A$ is used to normalize the above scheme so that $L_{d}$ is consistent with $L$. This yields $\kappa=1$ and $A=h^{-2}$. We obtain the symmetric 5-point stencil discretization operator

$$
L_{d,+}\left(E_{x}, E_{y}\right)=\frac{1}{h^{2}}\left[E_{x}^{-1}+E_{x}+E_{y}^{-1}+E_{y}-4 \cos \left(\frac{|\lambda|}{\sqrt{2}} h\right)\right]
$$

Rotating the above four coincident frequencies in the transform domain and the associated 5-point stencil in the space domain by an angle $\pi / 4$, we obtain another mode-dependent 5-point stencil discretization. In this scheme, the coincident frequencies become

$\left(\omega_{x}, \omega_{y}\right)=\left(|\lambda| \cos \left(\frac{n}{2} \pi\right),|\lambda| \sin \left(\frac{n}{2} \pi\right)\right), \quad 0 \leq n \leq 3$.

as shown in Fig. 1(b), and the resulting discretization operator is

$$
\begin{aligned}
L_{d, x}\left(E_{x}, E_{y}\right)= & \frac{1}{2 h^{2}}\left[E_{x}^{-1} E_{y}^{-1}+E_{x}^{-1} E_{y}+E_{x} E_{y}^{-1}\right. \\
& \left.+E_{x} E_{y}-4 \cos (|\lambda| h)\right] .
\end{aligned}
$$

Note that this rotated 5-point stencil can be viewed as corresponding to a discretization on a grid with spacing $\sqrt{2} h$. By appropriately combining (2.24), (2.25) and adding a constant term, we obtain the 9-point stencil discretization operator,

$$
\begin{aligned}
L_{d, 9}\left(E_{x}, E_{y}\right)= & \frac{\gamma_{x}}{\gamma_{x}+\gamma_{+}} L_{d,+}\left(E_{x}, E_{y}\right)+\frac{\gamma_{+}}{\gamma_{x}+\gamma_{+}} L_{d, x}\left(E_{x}, E_{y}\right) \\
& -\frac{\gamma_{x} \gamma_{+}}{\gamma_{x}+\gamma_{+}} .
\end{aligned}
$$

Then, if

$$
\begin{aligned}
\gamma_{\times} & =L_{d_{x}}\left(e^{i|\lambda| h / \sqrt{2}}, e^{j|\lambda| h / \sqrt{2}}\right) \\
& =\frac{1}{h^{2}}[\cos (\sqrt{2}|\lambda| h)+1-2 \cos (|\lambda| h)] \\
\gamma_{+} & =L_{d_{+}+}\left(e^{i|\lambda| h}, 1\right) \\
& =\frac{1}{h^{2}}\left[2 \cos (|\lambda| h)+2-4 \cos \left(\frac{|\lambda|}{\sqrt{2}} h\right)\right]
\end{aligned}
$$

we are able to match $L_{d}\left(z_{x}, z_{y}\right)$ and $L\left(s_{x}, s_{y}\right)$ at 8 frequencies $\left(\omega_{x}, \omega_{y}\right)=\left(|\lambda| \cos \left(\frac{n}{4} \pi\right),|\lambda| \sin \left(\frac{n}{4} \pi\right)\right), \quad 0 \leq n \leq 7$

as shown in Fig. 1(c). Thus, (2.26) is a mode-dependent 9-point stencil discretization operator for the Helmoltz equation. It can be shown that both $L_{h,+}$ and $L_{h, x}$ have an accuracy of $O\left(h^{2}\right)$ and the $L_{h, 9}$ has an accuracy of $O\left(h^{6}\right)$.
Convection-diffusion equation: In this case,

$$
L\left(D_{x}, D_{y}\right)=D_{x}^{2}+D_{y}^{2}-2 \alpha D_{x}-2 \beta D_{y}
$$

Then, if we consider only real frequencies $\left(s_{x}, s_{y}\right)=\left(\sigma_{x}, \sigma_{y}\right)$, the characteristic equation reduces to

$$
\sigma_{x}^{2}+\sigma_{y}^{2}-2 \alpha \sigma_{x}-2 \beta \sigma_{y}=0,
$$

which is a circle in the $\sigma_{x-\sigma y}$ plane centered at $(\alpha, \beta)$ with radius $d=\left(\alpha^{2}+\beta^{2}\right)^{7 / 2}$.

The conventional approach for discretizing the above equation uses central differences to approximate the first and second order derivatives separately. This gives

$$
\begin{aligned}
L_{d, c}\left(E_{x}, E_{y}\right)= & \frac{1}{h^{2}}\left[(1+\alpha h) E_{x}^{-1}+(1-\alpha h) E_{x}-4\right. \\
& \left.+(1+\beta h) E_{y}^{-1}+(1-\beta h) E_{y}\right]
\end{aligned}
$$

which corresponds to selecting a single coincident frequency at the origin. Allen and Southwell [3] combined two 1-D mode-dependent schemes, i.e., (2.13), along the $x$ - and $y$-directions. This yields

$$
\begin{aligned}
L_{d, A S}\left(E_{x}, E_{y}\right)= & \frac{1}{h}\left[\frac{2 \alpha}{\mathrm{e}^{2 \alpha h}-1}\left(\mathrm{e}^{2 \alpha h} E_{x}^{-1}-\left(1+\mathrm{e}^{2 \alpha h}\right)+E_{x}\right)\right. \\
& \left.+\frac{2 \beta}{\mathrm{e}^{2 \beta h}-1}\left(\mathrm{e}^{2 \beta h} E_{y}^{-1}-\left(1+\mathrm{e}^{2 \beta h}\right)+E_{y}\right)\right]
\end{aligned}
$$

which corresponds to selecting $(0,0),(2 \alpha, 0),(0,2 \beta),(2 \alpha, 2 \beta)$ as coincident frequencies. Motivated by the discussion of the previous section, we can also select the coincident frequencies

$$
\begin{aligned}
&\left(\sigma_{x}, \sigma_{y}\right)=\left(\alpha+d \cos \left(\frac{n}{2} \pi+\frac{1}{4} \pi\right),\right. \\
&\left.\beta+d \sin \left(\frac{n}{2} \pi+\frac{1}{4} \pi\right)\right), \quad 0 \leq n \leq 3
\end{aligned}
$$

uniformly along the contour (2.28), which gives the discretization operator

$$
\begin{aligned}
L_{d+}\left(E_{x}, E_{y}\right)= & \frac{1}{h^{2}}\left[\mathrm{e}^{\alpha h} E_{x}^{-1}+\mathrm{e}^{-\alpha h} E_{x}+\mathrm{e}^{\beta h} E_{y}^{-1}\right. \\
& \left.+\mathrm{e}^{-\beta h} E_{y}-4 \cosh \left(\frac{d}{\sqrt{2}} h\right)\right] .
\end{aligned}
$$

The multiplication of $E_{x}$ and $E_{y}$ by the factors $\mathrm{e}^{-\alpha h}$ and $\mathrm{e}^{-\beta h}$ in the space domain corresponds to a shift of the $s_{x}$ and $s_{y}$ variables in the transform domain, where $s_{x}$ and $s_{y}$ become $s_{x}-\alpha$ and $s_{y}-\beta$, respectively. The above scheme shifts therefore the center $(\alpha, \beta)$ of the circle (2.28) to the origin and interprets the resulting circle as corresponding to a Helmoltz equation with radius $d$. The coincident frequencies for the three schemes (2.29)-(2.31) are shown in Fig. 2. Following a procedure similar to the one used for the Helmoltz equation, we can also design mode-dependent rotated 5-point and 9-point stencil discretization schemes for the convection-diffusion equation. These schemes have an accuracy of $O\left(h^{2}\right)$ and $O\left(h^{6}\right)$, respectively.

\section{Historical Notes}

Historically, the idea of selecting exponential functions as coincident modes was first suggested by Allen and 


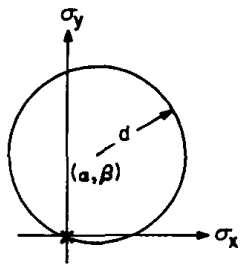

(a)

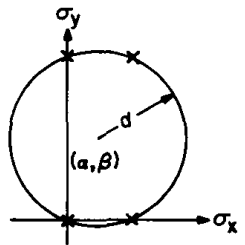

(b)

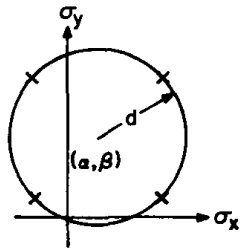

(c)

Fig. 2. Coincident frequencies of the (a) central difference, (b) Allen-Southwell, and (c) uniformly distributed modedependent 5-point discretizations of the convection-diffusion equation.

Southwell [3] for discretizing the convection-diffusion equation. An important feature of this problem is that there are large first-order terms in the governing second-order PDE. Due to these large first-order terms, there exists a boundary layer which cannot be well approximated by polynomials. The use of trigonometric functions as coincident modes was first discussed by Gautschi [45] for the numerical integration of ODEs which have periodic or oscillatory solutions whose periods can be estimated in advance. The advantage of selecting nonpolynomial functions as coincident modes has been recognized for years and applied to PDE problems repeatedly in the literature (see for example the references appearing in [72]). However, until recently, all mode-dependent discretization results were derived by considering one specific equation at a time, and it is only in [72] that a general framework was provided for the study of mode-dependent discretization methods.

III. Solution of Self-Adjoint Positive Definite Elliptic PDES: PROBLEM Formulation

Once (1.1) has been discretized with a finite-difference or finite-element scheme, the remaining task is to solve a system of linear difference equations of the form

$$
A u_{d}=f_{d}
$$

where $A$ is a sparse matrix, and $u_{d}$ and $f_{d}$ are discrete approximations of $u$ and $f$, respectively. Suppose that $u_{d}$ and $f_{d}$ are vectors of length $N$. The solution of (3.1) by Gaussian-elimination requires $O\left(N^{3}\right)$ operations, which is prohibitive for most practical applications. However, if the matrix $A$ is sym- metric positive definite (SPD), several direct and iterative methods [14], [57], [96], which require between $O(N)$ and $O\left(N^{2}\right)$ operations, can be used to solve (3.1) efficiently.

In the following, we shall restrict our attention to the case where the coefficient matrix $A$ in (3.1) is SPD. In terms of the differential operator (1.1), this amounts to second-order self-adjoint positive definite elliptic PDEs which can be expressed in the form

$$
\frac{\partial}{\partial x}\left(B \frac{\partial u}{\partial x}\right)+\frac{\partial}{\partial y}\left(C \frac{\partial u}{\partial y}\right)+D u=F
$$

where $B$ and $C$ are positive functions and $D \leq 0$. This subclass of equations includes the Poisson equation, which will be used below as the prototype for equations of the form (3.2).

To study the convergence rate of iterative solution techniques for (3.2), the traditional approach consists in using matrix iterative analysis [14], [57], [96], which relies on a detailed characterization of the structure of iteration matrices. Another approach, which has become popular recently, uses Fourier analysis to study the convergence behavior for a simple model problem. If the model problem is representative of the general class of problems that we want to solve, the convergence behavior for general problems can be inferred from the results obtained for the model problem. Since this second approach analyzes the effect of iterations on each Fourier mode through the use of digital signal processing methods, it is called here the DSP approach.

The advantage of the matrix approach is its general applicability. It can be applied to PDEs with irregular domain geometries, spatially varying coefficients, and when the discretization is performed on nonuniform grids. The only requirement is that the iteration matrices should possess certain properties, such as property $A$ or consistent ordering [57], [101], [102]. In contrast, the DSP approach can only be rigorously applied to a small class of problems. It presents, however, several advantages. First, the matrix approach is in general much more complicated than the DSP approach. Second, for simple problems, the DSP approach yields more accurate estimates of important quantities such as the optimal relaxation parameter for the SOR method, the smoothing rate of multigrid methods, or the eigenvalue distribution of the preconditioned operator obtained by applying a preconditioner to the discretized form of (3.2). Finally, the convergence behavior of iterative algorithms predicted by the DSP analysis of simple model problems is usually consistent with results obtained by performing numerical experiments on complicated problems. Thus, in spite of its simplicity, the DSP approach provides results which are applicable to very general problems.

\section{A. The Model Poisson Problem}

The standard model problem for (3.2) is the Poisson equation on the unit square $\Omega=[0,1]^{2}$

$$
\frac{\partial^{2} u(x, y)}{\partial x^{2}}+\frac{\partial^{2} u(x, y)}{\partial y^{2}}=f(x, y)
$$

with appropriate boundary conditions. It can be discretized on a uniform grid

$$
\Omega_{h}=\left\{\left(n_{x} h, n_{y} h\right): 0 \leq n_{x}, n_{y} \leq M\right\}
$$


with grid spacing $h=M^{-1}$. Approximating the Laplacian with the 5-point finite-difference scheme (2.19), and denoting by $u_{n_{x}, n_{\gamma}}$ the discrete approximation of the solution $u\left(n_{x} h, n_{y} h\right)$, we obtain the discretized system

$$
\begin{aligned}
& \frac{1}{h^{2}}\left(u_{n_{x}+1, n_{y}}+u_{n_{x}-1, n_{y}}+u_{n_{x}, n_{y}+1}\right. \\
& \left.\quad+u_{n_{x}, n_{y}-1}-4 u_{n_{x}, n_{y}}\right)=f_{n_{x}, n_{y}}
\end{aligned}
$$

at points $\left(n_{x} h, n_{y} h\right)$ which are located in the interior of $\Omega_{h}$ i.e., for $1 \leq n_{x}, n_{y} \leq M-1$. This system can be rewritten in terms of shift operators as

$$
A\left(E_{x}, E_{y}\right) u_{n_{x}, n_{y}}=-\frac{h^{2}}{4} f_{n_{x}, n_{y}}
$$

with

$$
A\left(E_{x}, E_{y}\right)=1-\frac{1}{4}\left(E_{x}+E_{x}^{-1}+E_{y}+E_{y}^{-1}\right) .
$$

Boundary Conditions: For self-adjoint positive definite elliptic PDEs, it has been observed empirically [24] that the convergence behavior of a given iterative algorithm is not significantly affected by the choice of boundary conditions. This implies that we can, without loss of rigor, restrict our attention to Dirichlet or periodic boundary conditions, since these boundary conditions have the advantage that they lend themselves easily to Fourier analysis. For Dirichlet boundary conditions, the solution $u(x, y)$ is specified along the boundary of the domain $\Omega$. In terms of the discretized system (3.5), this means that $u_{n_{x}, 0}, u_{n_{x}, M}, u_{0, n_{y}}$ and $u_{M, n_{y}}$ are given. Thus, the system (3.5) consists of $(M-1)^{2}$ equations in $(M-1)^{2}$ unknowns. Since nonzero boundary values can be moved to the right hand side and treated as part of the driving function, the system (3.5) with Dirichlet boundary conditions can be replaced by an equivalent system with a modified driving function and zero boundary conditions. Without loss of generality, the system (3.5) with zero boundary conditions

$$
u_{n_{x}, 0}=u_{n_{x}, M}=u_{0, n_{y}}=u_{M, n_{y}}=0
$$

where $1 \leq n_{x}, n_{y} \leq M-1$, is therefore called the model Dirichlet problem. Similarly, the system (3.5) with periodic boundary conditions

$$
u_{n_{x}, 0}=u_{n_{x}, M} \text { and } u_{0, n_{y}}=u_{M, n_{y}}
$$

where $0 \leq n_{x}, n_{y} \leq M-1$, is called the model periodic problem. It is easy to check that the model periodic problem involves $M^{2}$ equations in $M^{2}$ variables.

\section{B. Orderings}

To specify an algorithm for processing a multidimensional sequence, it is important to indicate the order in which the sequence should be computed. For example, a certain ordering of grid points is needed to implement 2D IIR filters. Similarly, for PDE algorithms, it is necessary to indicate clearly the ordering scheme which is employed, since the numetical performance of a given algorithm depends in general on the ordering [1], [70], [87]. We will focus our attention here on the natural and red-black orderings, since they are the most commonly employed, and are both amenable to Fourier analysis. The natural ordering corresponds to a standard rowwise (or columnwise) lexicographic ordering of the grid points. In the red- black ordering, the grid points are partitioned into two groups, which a grid point $\left(n_{x}, n_{y}\right)$ is red if $n_{x}+n_{y}$ is even, and black if $n_{x}+n_{y}$ is odd. Then, as a group, the red points precede the black points, but within each group, points are ordered according to the natural ordering.

Many PDE algorithms have the feature that numerical operations at a given point require only local information. In this case, it is usually possible to divide the grid points into subsets such that operations performed at points within a subset are independent of each other. In this case, the ordering of points within a subset is not important, since operations at such points can be implemented in parallel on a multiprocessor machine. When solving equation (3.5), this leads us to consider the following parallel versions of the natural and red-black orderings.

Parallel natural ordering:

$$
\left(n_{x}, n_{y}\right)<\left(m_{x}, m_{y}\right) \quad \text { if } n_{x}+n_{y}<m_{x}+m_{y} .
$$

\section{Parallel red-black ordering:}

$$
\left(n_{x}, n_{y}\right)<\left(m_{x}, m_{y}\right) \quad \text { if }\left(n_{x}, n_{y}\right) \text { red and }\left(m_{x}, m_{y}\right) \text { black. }
$$

In (3.10) and (3.11), the order between grid points is denoted by an inequality sign. Note that the above parallel natural ordering does not specify an order for points $\left(n_{x}, n_{y}\right)$ such that $n_{x}+n_{y}$ is constant. Similarly, for the parallel red-black ordering, no order is imposed for points of the same color. This is due to the fact that when the Gauss-Seidel or SOR methods described in Section $V$ below are used to solve (3.5), for the natural ordering, points along constant $n_{x}+$ $n_{y}$ lines can be updated in parallel. On the other hand, for the red-black version of the same relaxation methods, all points of identical color can be updated in parallel. From the point of view of parallelism, the red-black ordering is therefore preferable, since only two steps are required to scan all the grid points, instead of $O\left(N^{1 / 2}\right)$ steps for the natural ordering. However, the convergence rate of a given iterative algorithm can also be affected by the choice of ordering. For example, it has been shown recently [70] that the rate of convergence of the symmetric successive overrelaxation (SSOR) and of several preconditioned conjugate gradient methods can be slowed significantly if we use a red-black ordering instead of the natural ordering. Thus, when selecting a given ordering, one has to be careful to examine both the numerical complexity of the resulting algorithm as well as its parallelism.

\section{Fourier Analysis}

Several different Fourier basis functions will be introduced to expand $2 \mathrm{D}$ sequences. A sequence $u_{n_{x}, n_{y}}$ defined on $\Omega_{h}$ with zero boundary values can be expanded in a sinusoidal Fourier series of the form

$$
u_{n_{x}, n_{y}}=\sum_{k_{x}=1}^{M-1} \sum_{k_{y}=1}^{M-1} \hat{0}_{k_{x}, k_{y}} \sin \left(k_{x} \pi n_{x} h\right) \sin \left(k_{y} \pi n_{y} h\right) .
$$

It is easy to see that when $A\left(E_{x}, E_{y}\right)$ is given by (3.7), we have

$$
\begin{aligned}
& A\left(E_{x}, E_{y}\right) \sin \left(k_{x} \pi n_{x} h\right) \sin \left(k_{y} \pi n_{y} h\right) \\
& \quad=\hat{A}\left(k_{x}, k_{y}\right) \sin \left(k_{x} \pi n_{x} h\right) \sin \left(k_{y} \pi n_{y} h\right)
\end{aligned}
$$

with

$$
\hat{A}\left(k_{x}, k_{y}\right)=1-\frac{1}{2}\left[\cos \left(k_{x} \pi h\right)+\cos \left(k_{y} \pi h\right)\right] .
$$


Therefore, $\sin \left(k_{x} \pi n_{x} h\right) \sin \left(k_{y} \pi n_{y} h\right)$ is an eigenfunction of operator $A\left(E_{x}, E_{y}\right)$ corresponding to the eigenvalue $\hat{A}\left(k_{x}, k_{y}\right)$. It is worth noting at this point that by imposing the condition that the solution $u_{n_{x}, n_{y}}$ is synthesized by a finite number of Fourier sine functions as in (3.12), we are able to ignore the zero boundary conditions (3.8) for the model Dirichlet problem and treat $A\left(E_{x}, E_{y}\right)$ as a shift-invariant operator defined on an infinite grid.

Next, consider a sequence $u_{n x, n}$ defined on $\Omega_{h}$ which satisfies the periodic boundary conditions (3.9). The sequence $u_{n_{x}, n_{y}}$ can be expanded in complex exponential Fourier series as

$$
u_{n_{x}, n_{y}}=\sum_{k_{x}=0}^{M-1} \sum_{k_{y}=0}^{M-1} \hat{u}_{k_{x}, k_{y}} e^{\left.i 2 \pi i k_{x} n_{x}+k_{y} n_{y}\right) h}
$$

Since

$$
A\left(n_{x}, n_{y}\right) e^{i 2 \pi\left(k_{x} n_{x}+k_{y} n_{y} h h\right.}=\hat{A}\left(k_{x}, k_{y}\right) e^{i 2 \pi\left(k_{x} n_{x}+k_{y} n_{y}\right) h}
$$

where

$$
\hat{A}\left(k_{x}, k_{y}\right)=1-\frac{1}{2}\left[\cos \left(k_{x} 2 \pi h\right)+\cos \left(k_{y} 2 \pi h\right)\right]
$$

we see that $e^{i 2 \pi\left(k_{x} n_{x}+k_{y} n_{y}\right) h}$ is an eigenfunction of $A\left(E_{x}, E_{y}\right)$ with eigenvalue (3.17). Consequently, by expressing an arbitrary solution as a finite sum of such eigenfunctions, where $k_{x}$ and $k_{y}$ are integers between 0 and $M-1$, we can ignore the periodic boundary conditions (3.9) for the model periodic problem and view $A\left(E_{x}, E_{y}\right)$ as a shift-invariant operator defined on an infinite grid.

To analyze algorithms with a red-black ordering, we can employ a variant of the above Fourier decompositions, which is known as the two-color Fourier analysis [69], [70] Consider the model Dirichlet problem, and let $u_{n_{x}, n_{y}}$ be a sequence defined on $\Omega_{h}$ with zero boundary values. The restriction of this sequence to the red and black points defines two subsequences: the red sequence $u_{r, n_{x}, n_{y}}$ and the black sequence $u_{b, n_{x}, n_{y}}$. They can be expanded respectively in Fourier series as

$$
\begin{gathered}
u_{r, n_{x}, n_{y}}=\Sigma_{\left(k_{x}, k_{y}\right) \in K_{r}} \hat{u}_{r, k_{x}, k_{y}} \sin \left(k_{x} \pi n_{x} h\right) \sin \left(k_{y} \pi k h\right), \\
n_{x}+n_{y} \text { even } \\
u_{b, n_{x}, n_{y}}=\Sigma_{\left(k_{x}, k_{y}\right) \in K_{b}} \hat{u}_{b, k_{x}, k_{y}} \sin \left(k_{x} \pi n_{x} h\right) \sin \left(k_{y} \pi k h\right), \\
n_{x}+n_{y} \text { odd }
\end{gathered}
$$

where for $M$ even,

$$
\begin{gathered}
K_{b}=\left\{\left(k_{x}, k_{y}\right) \in N^{2}: k_{x}+k_{y} \leq M-1, k_{x}, k_{y} \geq 1\right. \text { or } \\
\left.1 \leq k_{x} \leq \frac{M}{2}-1, k_{y}=M-k_{x}\right\}
\end{gathered}
$$

and

$$
K_{r}=K_{b} \cup\{(M / 2, M / 2)\} .
$$

It is straightforward to check that the Fourier coefficients $\hat{u}_{k_{x}, k_{y}}, \hat{u}_{M-k_{x}, M-k_{y}}$ in the sinusoidal expansion (3.12) and $\hat{u}_{r, k_{x}, k_{y}}, \hat{u}_{b, k_{x}, k_{y}}$ in the red-black expansion (3.18) are related via

$$
\left(\begin{array}{l}
\hat{u}_{r, k_{x}, k_{y}} \\
\hat{u}_{b, k_{x}, k_{y}}
\end{array}\right)=\left[\begin{array}{cc}
1 & 1 \\
1 & -1
\end{array}\right]\left(\begin{array}{l}
\hat{u}_{k_{x}, k_{y}} \\
\hat{u}_{M-k_{x}, M-k_{y}}
\end{array}\right), \quad\left(k_{x}, k_{y}\right) \in K_{b}
$$

$$
\hat{u}_{r, k_{x}, k_{y}}=\hat{u}_{k_{x}, k_{y^{\prime}}} \quad\left(k_{x}, k_{y}\right)=(M / 2, M / 2) .
$$

The expression can be interpreted as follows. When the sequence $u_{n_{x}, n_{y}}$ is sampled only at the red points, instead of all points of $\Omega_{h}$, the high frequency component $\left(M-k_{x}\right.$, $\left.M-k_{y}\right)$ is aliased into the low frequency component $\left(k_{x}\right.$, $k_{y}$ ), so that two Fourier components coexist in the low frequency region. A similar aliasing phenomenon occurs when $u_{n_{x}, n_{y}}$ is sampled at the black points only (see Fig. 3 ). Note

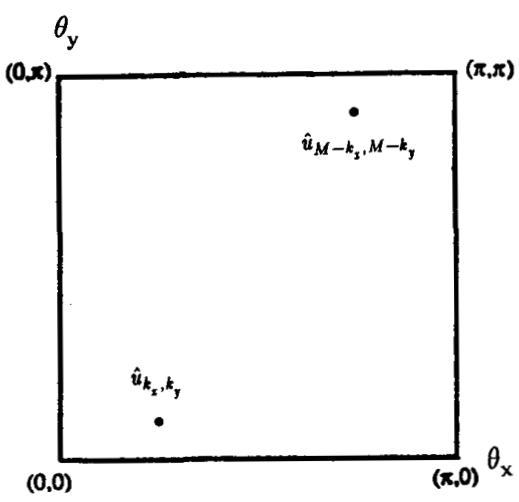

(a)

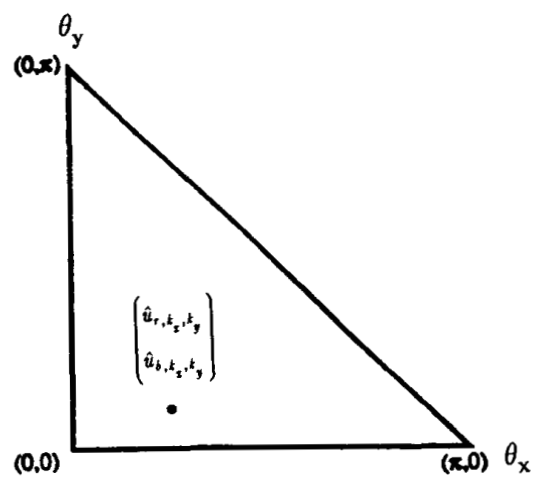

(b)

Fig. 3. (a) Conventional and (b) folded two-color Fourier domains where $\theta_{x}=k_{x} \pi h$ and $\theta_{y}=k_{y} \pi h$.

also that $K_{r}$ and $K_{b}$ differ by the single element $(M / 2, M / 2)$, so that at the frequency $(M / 2, M / 2)$ a single Fourier coefficient $\hat{u}_{r, M / 2, M / 2}$ is used to represent the $2 \mathrm{D}$ sequence $u_{n_{x}, n_{y}}$. This frequency can therefore be viewed as being degenerate.

With respect to the two-color decomposition (3.18), the discretized system (3.5) can be rewritten as

$$
A\left(E_{x}, E_{y}\right)\left(\begin{array}{l}
u_{r, n_{x}, n_{y}} \\
u_{b, n_{x}, n_{y}}
\end{array}\right)=-\frac{h^{2}}{4}\left(\begin{array}{l}
f_{r, n_{x}, n_{y}} \\
f_{b, n_{x}, n_{y}}
\end{array}\right)
$$

with

$$
A\left(E_{x}, E_{y}\right)=\left[\begin{array}{cc}
1 & -a\left(E_{x}, E_{y}\right) \\
-a\left(E_{x}, E_{y}\right) & 1
\end{array}\right]
$$

and

$$
a\left(E_{x}, E_{y}\right)=\frac{1}{4}\left(E_{x}+E_{x}^{-1}+E_{y}+E_{y}^{-1}\right) .
$$


To obtain a frequency domain representation of the above system, we can substitute the Fourier decomposition (3.18) inside (3.21) and match Fourier components. For a nondegenerate frequency $\left(k_{x}, k_{y}\right)$, this gives

$$
\hat{A}\left(k_{x}, k_{y}\right)\left(\begin{array}{l}
u_{r, k_{x}, k_{y}} \\
u_{b, k_{x}, k_{y}}
\end{array}\right)=-\frac{h^{2}}{4}\left(\begin{array}{l}
\hat{f}_{r, k_{x}, k_{y}} \\
\hat{f}_{b, k_{x}, k_{y}}
\end{array}\right)
$$

with

$$
\hat{A}\left(k_{x}, k_{y}\right)=\left[\begin{array}{cc}
1 & -\hat{a}\left(k_{x}, k_{y}\right) \\
-\hat{a}\left(k_{x}, k_{y}\right) & 1
\end{array}\right]
$$

and where

$$
\hat{\mathrm{a}}\left(k_{\mathrm{x}}, k_{y}\right)=\frac{1}{2}\left[\cos \left(k_{\mathrm{x}} \pi h\right)+\cos \left(k_{y} \pi h\right)\right]
$$

is the Fourier transform of the space domain operator $A\left(E_{x}\right.$, $\left.E_{y}\right)$. For the degenerate frequency $\left(k_{x}, k_{y}\right)=(M / 2, M / 2)$, we obtain

$$
\hat{u}_{r, M / 2, M / 2}=-\frac{h^{2}}{4} \hat{f}_{r, M / 2, M / 2}
$$

Note that the above results rely in part on the fact that for the Dirichlet case, the eigenfunctions of the $2 \times 2$ matrix operator $A\left(E_{x}, E_{y}\right)$ are of the form $v\left(k_{x}, k_{y}\right) \sin \left(k_{x} \pi n_{x} h\right) \sin$ $\left(k_{y} \pi n_{y} h\right)$ where the 2-vector $v\left(k_{x}, k_{y}\right)$ is an eigenvector of the matrix $\hat{A}\left(k_{x}, k_{y}\right)$.

In the previous two-color Fourier analysis of the red-black ordering, we have assumed that the boundary conditions are of Dirichlet type. For the case of periodic boundary conditions, a similar two-color Fourier analysis can be developed. One needs only to replace the sinusoidal expansions (3.18) by complex exponential Fourier series. Since the analysis is identical to the Dirichlet case, the details are omitted. We find that identities (3.21)-(3.25) remain valid, provided that the function $\hat{a}\left(k_{x}, k_{y}\right)$ is replaced by

$$
\hat{\mathrm{a}}\left(k_{x}, k_{y}\right)=\frac{1}{2}\left[\cos \left(k_{x} 2 \pi h\right)+\cos \left(k_{y} 2 \pi h\right)\right] \text {. }
$$

\section{Summary}

In this section, we have examined the model Poisson problem with Dirichlet or periodic boundary conditions, and with a natural or red-black ordering. In each case, a Fourier basis has been introduced to expand 2D sequences satisfying the boundary conditions. For such sequences, it has been shown that the system (3.5) can be viewed as a linear shift-invariant (LSI) system in the space domain, and can therefore be analyzed in the frequency domain. The results of our analysis are summarized in Table 1.

The Fourier analysis that we have developed in this section has focused on the operator $A\left(E_{x}, E_{y}\right)$ defined in (3.7)

Table 1 Fourier Decomposition for Several Orderings and Boundary Conditions

\begin{tabular}{llccc}
\hline \hline Ordering & B.C. & $A\left(E_{x}, E_{y}\right)$ & $\begin{array}{c}\text { Fourier basis } \\
\text { functions }\end{array}$ & $\hat{A}\left(k_{x}, k_{y}\right)$ \\
\hline natural & Dirichlet & $(3.7)$ & $(3.12)$ & $(3.14)$ \\
natural & periodic & $(3.7)$ & $(3.15)$ & $(3.17)$ \\
red-black & Dirichlet & $(3.22)$ & $(3.18)$ & $(3.25),(3.26)$ \\
red-black & periodic & $(3.22)$ & $\begin{array}{c}\text { r-b complex } \\
\text { sinusoids }\end{array}$ & $(3.25),(3.27)$ \\
\hline
\end{tabular}

or (3.22). Since this operator is an FIR filter, the ordering of grid points does not play a role in its implementation, so that as $\operatorname{far}$ as $A$ is concerned, the distinction between the natural and red-black orderings is really unnecessary. However, when solving (3.5), our actual goal is to implement the inverse filter $A^{-1}\left(E_{x}, E_{y}\right)$, which is a $2 \mathrm{D}$ IIR filter, and for which the choice of ordering does matter. To synthesize this filter, we will rely on the iterated application of deconvolution filters, which will be in general of 2D IIR type, thus explaining our interest in the choice of ordering.

\section{DIRECT METHODS}

Several efficient direct methods have been developed for solving elliptic PDEs. These methods usually exploit special features of certain classes of PDEs, and are often restricted to regular domain geometries. They are therefore not as widely applicable as the iterative methods to be discussed in the following sections. Furthermore, except for fast Fourier solvers, direct methods rely mainly on matrix or graphtheoretic techniques. Thus, they do not fit well the DSP viewpoint adopted in this paper. Consequently, in this section we focus primarily our attention on FFT solvers. However, for completeness, several other direct methods, such as cyclic block-reduction and sparse Gaussian elimination methods, are briefly discussed.

\section{A. FFT Solvers}

Fast Fourier solvers are applicable to 2-D separable elliptic PDEs of the form

$$
(P(x)+Q(y)) u(x, y)=f(x, y)
$$

defined on the unit square $[0,1]^{2}$, with

$$
\begin{aligned}
P(x) & =\frac{\partial}{\partial x}\left(p_{1}(x) \frac{\partial}{\partial x}\right)+p_{2}(x) \\
Q(y) & =\frac{\partial}{\partial y}\left(q_{1}(y) \frac{\partial}{\partial y}\right)+q_{2}(y)
\end{aligned}
$$

and where $p_{1}(x) q_{1}(y)>0$. For simplicity, we assume that the boundary conditions are of Dirichlet type, i.e., $u(x, y)$ $=0$ on the domain boundary. A wider class of boundary conditions is considered in [94].

By discretizing the differential operators $P(x)$ and $Q(y)$ on a uniform $\Omega_{h}$ with spacing $h=M^{-1}$, with 3-point central diferences in the $x$-and $y$-directions, respectively, we obtain a 5-point stencil discretization of (4.1). The discretized system can be denoted as

$$
\left(P_{d}\left(n_{x}\right)+Q_{d}\left(n_{y}\right)\right) u_{n_{x}, n_{y}}=f_{n_{x}, n_{y}} .
$$

FFT solvers require that either $P(x)$ or $Q(y)$ should have constant coefficients. If the coefficients $p_{1}(x)=p_{1}$ and $p_{2}(x)$ $=p_{2}$ of $P(x)$ are constant, the discretized operator

$$
P_{d}\left(n_{x}\right)=P_{d}=\frac{p_{1}}{h^{2}}\left(E_{x}-2+E_{x}^{-1}\right)+p_{2}
$$

has also constant coefficients. Then, the Fourier transform can be used to transform the discretized equation (4.3), which depends on the two variables $n_{x}$ and $n_{y}$, into a set of decoupled equations depending on the single variable $n_{y}$. Specifically, due to the separability of equation (4.1), we can express the solution $u_{n_{x}, n_{y}}$ and driving function $f_{n_{x}, n_{y}}$ in the 
form

$$
\begin{aligned}
u_{n_{x}, n_{y}} & =\sum_{k_{x}=1}^{M-1} \hat{u}_{k_{x}, n_{y}} \sin \left(k_{x} \pi n_{x} h\right), \\
f_{n_{x}, n_{y}} & =\sum_{k_{x}=1}^{M-1} \hat{f}_{k_{x}, n_{y}} \sin \left(k_{x} \pi n_{x} h\right) .
\end{aligned}
$$

Substituting (4.5) into (4.3), we obtain $M-1$ independent equations

$$
\left(\hat{P}_{d}+Q_{d}\left(n_{y}\right)\right) \hat{u}_{k_{x}, n_{y}}=\hat{f}_{k_{x}, n_{y},} \quad 1 \leq k_{x} \leq M-1
$$

with

$$
\hat{P}_{d}=-\frac{2 p_{1}}{h^{2}}\left[1-\cos \left(k_{x} \pi h\right)\right]+p_{2} .
$$

The boundary conditions of the transformed system are also of Dirichlet type, i.e.,

$$
\hat{u}_{k_{x}, 0}=\hat{u}_{k_{x}, M}=0
$$

Then, for each value of $k_{x}$, the system (4.6)-(4.7) can be written in matrix form as a tridiagonal system

$\left[\begin{array}{cccccc}a_{1} & c_{1} & & & & \\ b_{2} & a_{2} & c_{2} & & & \\ & \cdot & \cdot & \cdot & & \\ & & \cdot & \cdot & \cdot & \\ & & & b_{M-2} & a_{M-2} & c_{M-2} \\ & & & & b_{M-1} & a_{M-1}\end{array}\right]\left[\begin{array}{c}\hat{u}_{1} \\ \hat{u}_{2} \\ \cdot \\ \cdot \\ \hat{u}_{M-2} \\ \hat{u}_{M-1}\end{array}\right]=\left[\begin{array}{c}\hat{f}_{1} \\ \hat{f}_{2} \\ \cdot \\ \cdot \\ \hat{f}_{M-2} \\ \hat{f}_{M-1} \\ (4.8)\end{array}\right]$

(4.8)

where the $k_{x}$ dependence of the solution, driving term, and matrix entries has been suppressed. Each such system can be solved directly with the following algorithm of complexity $O(M)$ (Table 2).

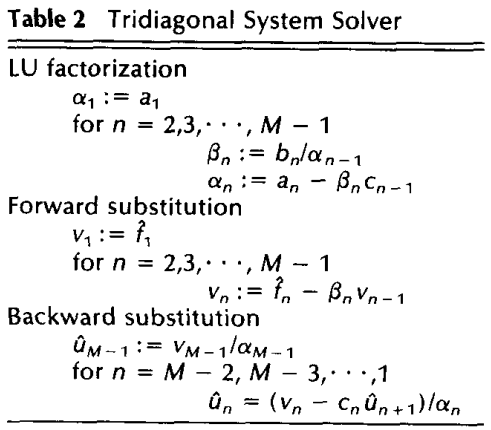

Given the solutions $\hat{u}_{k_{x}, n_{y}}$ of systems (4.6)-(4.7) for all $k_{x}$, the solution $u_{n_{x}, n_{y}}$ of the PDE can then be obtained from the discrete sine transform (4.5).

Fast Fourier solvers rely therefore on the following three steps.

Step 1: Perform a 1-D fast sine transform of $f_{n_{x} n_{y}}$ with respect to $n_{x}$ to determine the hybrid Fourier coefficients $\hat{f}_{k_{x}, n_{y}}$.

Step 2: For each $k_{x}$, with $1 \leq k_{x} \leq M-1$, calculate the hybrid Fourier coefficients $\hat{u}_{k_{x}, n_{y}}$ by solving the tridiagonal system corresponding to (4.6)-(4.7).
Step 3: Perform a 1-D fast inverse sine transform to compute the solution $u_{n_{x}, n_{y}}$ from the hybrid Fourier coefficients $\hat{u}_{k_{x}, n_{y}}$.

In the above discussion, we have assumed that the boundary conditions are of Dirichlet type. However, other choices of boundary conditions, such as Neumann or periodic conditions, are also possible. The effect of a change of boundary conditions is to replace the fast sine transform in steps 1) and 3) above by fast cosine transforms, or FFTs [93], [94]. The complexity of the resulting family of FFT solvers is $O\left(M^{2} \log (M)\right)$. However, it is important to keep in mind that these solvers are restricted to problems with a rectangular domain, and where either $P(x)$ or $Q(y)$ has constant coefficients.

\section{B. Other Direct Methods}

The above FFT solver was introduced by Hockney in 1965 [59] for the Poisson problem over a rectangle. In both [59] and [60] Hockney discussed another direct method, called cyclic reduction. This method is a Gaussian elimination procedure with a particular ordering. Specifically, assume that in (4.3), the variables $u_{n_{x}, n_{y}}$ are scanned column by column, and let $u_{n}$ be the $M-1$ dimensional vector formed by the variables with column index $n_{x}$. It is easy to check that the set of vectors $u_{n_{x}}$ with $1 \leq n_{x} \leq M-1$ satisfies a block tridiagonal system. Then, assume that we eliminate one out of every two columns from this system, say the columns with $n_{x}$ even. The resulting system remains block tridiagonal, although the blocks may start to fill in. By proceeding recursively, after $L=\log (M)$ steps, a single column of variables remains. The resulting system of size $M-1$ can be solved, and its solution can be backsubstituted into the system obtained at the previous level, thus enabling us to compute progressively all columns of the original system. For more details on the cyclic block reduction method, the reader is referred to [93], [94]. This method which was unstable in its original form, was later stabilized by Buneman [22]. The complexity of the resulting procedure is $\mathrm{O}\left(\mathrm{M}^{2} \log \right.$ (M)), as for FFT solvers.

The FFT and cyclic block reduction methods can be combined to produce a third technique, called the Fourier analysis-cyclic reduction (FACR) algorithm, whose complexity is $O\left(M^{2} \log \log (M)\right)$. The FFT, cyclic reduction and FACR solvers are reviewed by Swarztrauber in [93]. Another survey [94] provides a more elementary introduction to this topic.

The cyclic block reduction procedure can be viewed as a special case of a more general family of direct solvers, called sparse Gaussian elimination methods. These methods start from a system of the form

$$
A x=b
$$

where $A$ is symmetric positive definite. The matrix $A$ is usually sparse. However, when (4.9) is solved by performing a Cholesky factorization $A=L L^{T}$, the lower triangular matrix $L$ contains in general more nonzero elements than existed in the lower triangular part of $A$, thus resulting in an increase in the storage and computation time required to solve (4.9) by Gaussian elimination. However, the amount of fill, i.e. the number of additional nonzero entries of $L$, depends highly on the ordering of the variables. If $P$ denotes an arbitrary permutation matrix, it may be of interest to replace the 
solution of (4.9) by that of

$$
B y=c
$$

with

$$
B=P A P^{T} ; y=P x ; c=P b .
$$

An ordering is said to be optimal with respect to fill, if it results in the least possible fill-in, and optimal with respect to operation count if it minimizes the number of operations required to solve (4.10a) by Gaussian elimination. If $A$ is an $N \times N$ matrix, there are $N$ ! different orderings of its rows and columns, and the problem of finding the ordering with least fill-in is NP complete. Efforts have therefore focused on obtaining efficient algorithms for finding suboptimal orderings with low fill-in and operation count. Numerous reordering algorithms have been developed based on results from graph theory. This topic is discussed in detail in books by George and Liu [46], and Duff, Erisman, and Reid [35].

\section{Relaxation Methods and Their acceleration}

A general mechanism for constructing iterative algorithms for the solution of discretized elliptic PDEs consists of using relaxation. In this approach, instead of requiring that the entire system (3.1) of discretized equations should be satisfied, we force only one or a few equations to hold at any given time. For the case of a single equation, the value of the variable $u_{n_{x}, n_{y}}$ is updated by forcing the discretization equation to hold at point $\left(n_{x} h, n_{y} h\right)$, while relaxing it at all other points of the discretization grid $\Omega_{h}$. By using this procedure sequentially, or if possible in parallel, for all points of $\Omega_{h}$, an updated value of the solution is obtained at all grid points, and one can then proceed to the next iteration. If the resulting iterative algorithm converges, the complete system (3.1) of discretized equations will eventually be satisifed.

In this section, we describe elementary relaxation methods, such as the Jacobi and Gauss-Seidel iterations, and use a digital filtering viewpoint to analyze their convergence behavior. The major shortcoming of these methods is their slow convergence rate. Several acceleration schemes have been proposed to improve their convergence. Acceleration schemes can be divided into two categories, depending on whether they are stationary or not. In a stationary scheme, the same acceleration procedure is used at each iteration. Thus, we can focus on a single iteration and try to optimize its performance. The best example of such a procedure is the successive over-relaxation (SOR) method. In a nonstationary scheme, the overall performance of the algorithm is optimized by considering more than one iteration at a time. Examples of such schemes include the Chebyshev semi-iterative (CSI) and conjugate gradient (CG) methods. Both stationary and nonstationary acceleration methods are discussed below.

\section{A. Elementary Relaxation Methods}

Consider the discretization (3.5) of the model Poisson problem. The Jacobi relaxation is given by

$$
\begin{aligned}
u_{n_{x} n_{y}}^{(m+1)}= & \frac{1}{4}\left(u_{n_{x}+1, n_{y}}^{(m)}+u_{n_{x}-1, n_{y}}^{(m)}+u_{n_{x}, n_{y}+1}^{(m)}\right. \\
& \left.+u_{n_{x}, n_{y}-1}^{(m)}-h^{2} f_{n_{x}, n_{y}}\right)
\end{aligned}
$$

where $u_{n_{x}, n_{y}}^{(m)}$ denotes the value of the variable $u_{n_{x}, n_{y}}$ at the $m$ th iteration, with $m=0,1,2, \cdots$. From (5.1), we see that given the values $u_{n_{x}, n_{y}}^{(m)}$ at all points of $\Omega_{h}$, the value $u_{n_{x}, n_{y}}^{(m+1)}$ at the next iteration is obtained by forcing equation (3.5) to be locally satisfied at $\left(n_{x} h, n_{y} h\right)$, independently of whether it is violated at other points of $\Omega_{h}$.

One way to modify the Jacobi relaxation (5.1) is to partition the grid points into red and black two groups as described in Section III and to perform the iteration $\left(n_{x}, n_{y}\right)$ red:

$$
\begin{aligned}
u_{n_{x}, n_{y}}^{(m+1)}= & \frac{1}{4}\left(u_{n_{x}+1, n_{y}}^{(m)}+u_{n_{x}-1, n_{y}}^{(m)}+u_{n_{x}, n_{y}+1}^{(m)}\right. \\
& \left.+u_{n_{x}, n_{y}-1}^{(m)}-h^{2} f_{n_{x}, n_{y}}\right)
\end{aligned}
$$

$\left(n_{x}, n_{y}\right)$ black:

$$
\begin{aligned}
u_{n_{x}, n_{y}}^{(m+1)}= & \frac{1}{4}\left(u_{n_{x}+1, n_{y}}^{(m+1)}+u_{n_{x}-1, n_{y}}^{(m+1)}+u_{n_{x}, n_{y}+1}^{(m+1)}\right. \\
& \left.+u_{n_{x}, n_{y}-1}^{(m+1)}-h^{2} f_{n_{x}, n_{y}}\right) .
\end{aligned}
$$

Thus, one iteration consists of two steps. In the first step, a Jacobi relaxation is performed at all the red points and in the second step, the values obtained at the red points in the first step are used to perform a Jacobi relaxation at the black points. The iteration (5.2) is known as the Gauss-Seidel relaxation for the red-black ordering. The reader is referred to [75] for a detailed comparison of the red-black Gauss-Seidel and Jacobi relaxations.

To analyze the convergence behavior of relaxation methods, it is convenient to view each iteration as corresponding to a digital filtering operation on the solution error. For example, if the Jacobi relaxation converges, the iteration equation (5.1) reduces asymptotically to

$\bar{u}_{n_{x}, n_{y}}=\frac{1}{4}\left(\bar{u}_{n_{x}+1, n_{y}}+\bar{u}_{n_{x}-1, n_{y}}+\bar{u}_{n_{x}, n_{y}+1}+\bar{u}_{n_{x}, n_{y}-1}-h^{2} f_{n_{x}, n_{y}}\right)$

where $\bar{u}_{n_{x}, n_{y}}$ is the exact solution of the discretized problem. Subtracting (5.3) from (5.1), we find that the errors evolve according to

$$
e_{n_{x}, n_{y}}^{(m+1)}=\frac{1}{4}\left(E_{x}+E_{x}^{-1}+E_{y}+E_{y}^{-1}\right) e_{n_{x}, n_{y}}^{(m)}
$$

where

$$
\mathrm{e}_{n_{x}, n_{y}}^{(m)}=u_{n_{x}, n_{y}}^{(m)}-\bar{u}_{n_{x}, n_{y}}
$$

is the error at the $m$ th iteration. Thus, the Jacobi relaxation can be viewed as a digital filtering process, where at each iteration the FIR filter

$$
\mathcal{J}\left(E_{x}, E_{y}\right)=\frac{1}{4}\left(E_{x}+E_{x}^{-1}+E_{y}+E_{y}^{-1}\right)
$$

is applied to the errors obtained at the previous iteration. Assume that the boundary conditions for the Poisson problem are of Dirichlet type, so that the errors are zero on the domain boundary. To analyze (5.4) in the Fourier domain, we observe that the functions

$$
\sin \left(k_{x} \pi n_{x} h\right) \sin \left(k_{y} \pi n_{y} h\right), \quad 1 \leq k_{x}, k_{y} \leq M-1
$$

with $M=h^{-1}$, are eigenfunctions of $/$ which are zero on the domain boundary. They can therefore be used to expand the errors $e_{n_{x}, n_{y}}^{(m)}$ in the form (3.12). In the Fourier domain, the iteration (5.4) is diagonalized and takes the form

$$
\hat{e}_{k_{x}, k_{y}}^{(m+1)}=\hat{\jmath}\left(k_{x}, k_{y}\right) \hat{e}_{k_{x}, k_{y}}^{(m)}
$$


where the eigenvalues

$$
\hat{\jmath}\left(k_{x}, k_{y}\right)=\frac{1}{2}\left[\cos \left(k_{x} \pi h\right)+\cos \left(k_{y} \pi h\right)\right]
$$

specify the spectrum of $J$. The spectrum magnitude $\mid \hat{\jmath}\left(k_{x}\right.$ $\left.k_{y}\right) \mid$ is plotted in Fig. 4. We see from this figure that the Jacobi

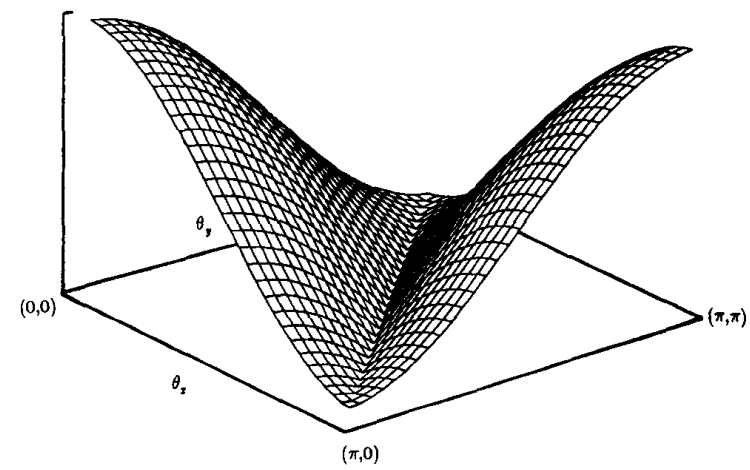

Fig. 4. The spectrum magnitude of the lacobi iteration operator.

relaxation acts as a notch filter. It filters out the middle frequencies, but dampens only slightly the low and high frequencies. Since $\left|\hat{j}\left(k_{x}, k_{y}\right)\right|<1$ for all feasible wavenumbers, the Jacobi relaxation converges. Its convergence rate is determined by the spectral radius

$$
\rho(J)=\max _{1 \leq k_{x}, k_{y} \leq M-1}\left|\hat{J}\left(k_{x}, k_{y}\right)\right|=\cos (\pi h) \approx 1-\frac{1}{2} \pi^{2} h^{2} .
$$

We see from (5.9) that the number of Jacobi iterations required to reduce the error by a constant factor is proportional to $O\left(h^{-2}\right)$. In order to determine the total number of iterations needed for convergence, it is useful to observe that since the discretized system is only an approximation of the original continuous problem, the iteration can be stopped when the solution error for the discretized system is of the same order as the discretization error. We saw in Section II-C that the error for a 5-point discretization of the Laplacian is $O\left(h^{2}\right)$. The total number of iterations required by the Jacobi relaxation is therefore $O\left(h^{-2} \log \left(h^{-1}\right)\right)$.

Similarly, denoting by $e_{r, n_{x}, n_{y}}^{(m)}$ and $e_{b, n_{x}, n_{y}}^{(m)}$ the restriction of the error at the $m$-th iteration to the red and black points, respectively, we find that the errors for the red-black Gauss-Seidel relaxation evolve according to

$$
\left(\begin{array}{l}
e_{r, n_{x}, n_{y}}^{(m+1)} \\
e_{b, n_{x}, n_{y}}^{(m+1)}
\end{array}\right)=G_{r b}\left(\begin{array}{l}
e_{r, n_{x}, n_{y}}^{(m)} \\
e_{b, n_{x}, n_{y}}^{(m)}
\end{array}\right)
$$

where

$$
G_{\mathrm{rb}}=\left[\begin{array}{ll}
1 & 0 \\
J & 0
\end{array}\right]\left[\begin{array}{ll}
0 & J \\
0 & 1
\end{array}\right]=\left[\begin{array}{ll}
0 & J \\
0 & J^{2}
\end{array}\right]
$$

is the red-black Gauss-Seidel relaxation operator. For Dirichlet boundary conditions, the red and black error functions admit a Fourier decomposition of the form (3.18). With respect to this basis, the error dynamics (5.10) decouples into a set of $2 \times 2$ matrix equations

$$
\begin{aligned}
& \left(\begin{array}{c}
\hat{e}_{r, k_{x}, k_{y}}^{(m+1)} \\
\hat{e}_{b, k_{x}, k_{y}}^{(m+1)}
\end{array}\right)=\hat{G}_{\mathrm{rb}}\left(k_{x}, k_{y}\right)\left(\begin{array}{c}
\hat{e}_{r, k_{x}, k_{y}}^{(m)} \\
\hat{e}_{b, k_{x}, k_{y}}^{(m)}
\end{array}\right) \\
& \hat{G}_{\mathrm{rb}}\left(k_{x}, k_{y}\right)=\left[\begin{array}{ll}
0 & \hat{j}\left(k_{x}, k_{y}\right) \\
0 & \hat{j}^{2}\left(k_{x}, k_{y}\right)
\end{array}\right]
\end{aligned}
$$

with $\left(k_{x}, k_{y}\right) \in K_{b}$, where $K_{b}$ is defined in (3.19a). The spectral radius of $G_{\mathrm{rb}}$ is therefore given by

$$
\rho\left(C_{\mathrm{rb}}\right)=\max _{k_{x}, k_{y} \in K_{b}}\left|\hat{J}^{2}\left(k_{x}, k_{y}\right)\right|=\cos ^{2}(\pi h) \approx 1-\pi^{2} h^{2} .
$$

Comparing (5.9) and (5.12), we see immediately that the convergence rate of the red-black Gauss-Seidel algorithm is double that of the Jacobi relaxation. Since both algorithms require the same number of operations per iteration, the red-black Gauss-Seidel algorithm is twice as efficient.

If the natural ordering is adopted, the Gauss-Seidel relaxation takes the form

$$
\begin{aligned}
u_{n_{x}, n_{y}}^{(m+1)}= & \frac{1}{4}\left(u_{n_{x}+1, n_{y}}^{(m)}+u_{n_{x}-1, n_{y}}^{(m+1)}+u_{n_{x}, n_{y}+1}^{(m)}\right. \\
& \left.+u_{n_{x}, n_{y}-1}^{(m+1)}-h^{2} f_{n_{x}, n_{y}}\right)
\end{aligned}
$$

and is called the lexicographic Gauss-Seidel iteration. The errors dynamics are given by

$$
e_{n_{x}, n_{y}}^{(m+1)}=C_{l e x} e_{n_{x}, n_{y}}^{(m)}
$$

where

$$
G_{\text {lex }}=\frac{E_{\mathrm{x}}+E_{\mathrm{y}}}{4-E_{x}^{-1}-E_{y}^{-1}}
$$

is a causal IIR filter. The spectral analysis of the operator $G_{\text {lex }}$ with Dirichlet boundary conditions has been performed by Frankel [42], and was studied by Trefethen and LeVeque [77] from a tilted grid viewpoint. For convenience, we consider here the case of periodic boundary conditions. Then, the eigenfunctions of $G_{\text {lex }}$ are

$$
\mathrm{e}^{i 2 \pi\left(k_{x} n_{x}+k_{y} n_{y}\right) h}, \quad 0 \leq k_{x}, k_{y} \leq M-1
$$

and, decomposing the errors with respect to this basis, the spectrum of $G_{\text {lex }}$ is given by

$$
\hat{G}_{\text {lex }}\left(k_{x}, k_{y}\right)=\frac{\mathrm{e}^{i 2 \pi k_{x} h}+\mathrm{e}^{i 2 \pi k_{y} h}}{4-\mathrm{e}^{-i 2 \pi k_{x} h}-\mathrm{e}^{-i 2 \pi k_{y} h}} .
$$

Note that $\hat{C}_{\text {lex }}\left(k_{x}, k_{y}\right)=1$ for $\left(k_{x}, k_{y}\right)=(0,0)$ and $\left|\hat{C}_{\text {lex }}\left(k_{x}, k_{y}\right)\right|$ $<1$ for all other feasible wavenumbers. This means that the filter $G_{\text {lex }}$ does not filter out the d.c. component of the error. However, if $u(x, y)$ is a solution of the model periodic problem, $u(x, y)$ plus a constant is also a solution, and the lexicographic Gauss-Seidel method converges to one of these solutions.

To summarize, the Jacobi, red-black, and lexicographic Gauss-Seidel relaxations admit a digital filtering interpretation, where each iteration consists in applying a filter to the errors obtained at the previous iteration. This filtering process can be studied easily in the frequency domain, by decomposing the errors in terms of properly selected Fourier eigenmodes, and examining each mode independently. 


\section{B. SOR Acceleration}

The red-black SOR iteration is obtained by introducing a relaxation parameter $\omega$ inside the Gauss-Seidel iteration (5.2), i.e.,

$\left(n_{x}, n_{y}\right)$ red:

$$
\begin{aligned}
u_{n_{x}, n_{y}}^{(m+1)}= & (1-\omega) u_{n_{x}, n_{y}}^{(m)}+\frac{\omega}{4}\left(u_{n_{x}+1, n_{y}}^{(m)}+u_{n_{x}-1, n_{y}}^{(m)}\right. \\
& \left.+u_{n_{x}, n_{y}+1}^{(m)}+u_{n_{x}, n_{y}-1}^{(m)}-h^{2} f_{n_{x}, n_{y}}\right)
\end{aligned}
$$

$\left(n_{x}, n_{y}\right)$ black:

$$
\begin{aligned}
u_{n_{x}, n_{y}}^{(m+1)}= & (1-\omega) u_{n_{x}, n_{y}}^{(m)}+\frac{\omega}{4}\left(u_{n_{x}+1, n_{y}}^{(m+1)}+u_{n_{x}-1, n_{y}}^{(m+1)}\right. \\
& \left.+u_{n_{x}, n_{y}+1}^{(m+1)}+u_{n_{x}, n_{y}-1}^{(m+1)}-h^{2} f_{n_{x}, n_{y}}\right) .
\end{aligned}
$$

When $\omega=1$, the SOR method reduces to the Gauss-Seidel method. The error dynamics for the SOR iteration can be expressed as

$$
\left(\begin{array}{c}
e_{t, n_{x}, n_{y}}^{(m+1)} \\
e_{b, n_{x}, n_{y}}^{(m+1)}
\end{array}\right)=C_{\mathrm{rb}}(\omega)\left(\begin{array}{l}
\mathbf{e}_{t, n_{x}, n_{y}}^{(m)} \\
\mathbf{e}_{b, n_{x}, n_{y}}^{(m)}
\end{array}\right)
$$

where

$$
\begin{aligned}
C_{\mathrm{rb}}(\omega) & =\left[\begin{array}{ll}
1 & 0 \\
\omega / & 1-\omega
\end{array}\right]\left[\begin{array}{ll}
1-\omega & \omega J \\
0 & 1
\end{array}\right] \\
& =\left[\begin{array}{ll}
1-\omega & \omega J \\
(1-\omega) \omega J & 1-\omega+\omega^{2} J^{2}
\end{array}\right]
\end{aligned}
$$

is the red-black SOR iteration operator. With respect to the red-black Fourier decomposition (3.18), the SOR iteration reduces to $2 \times 2$ matrix iterations of the form (5.11a), where $\hat{\mathrm{G}}_{\mathrm{rb}}\left(k_{\mathrm{x}}, k_{\mathrm{y}}\right)$ is replaced by

$$
\hat{G}_{\mathrm{rb}}\left(\omega, k_{\mathrm{x}}, k_{y}\right)=\left[\begin{array}{ll}
1-\omega & \omega \hat{\jmath}\left(k_{x}, k_{y}\right) \\
(1-\omega) \omega \hat{\jmath}\left(k_{x}, k_{y}\right) & 1-\omega+\omega^{2} \hat{J}^{2}\left(k_{x}, k_{y}\right)
\end{array}\right] .
$$

Let $\lambda$ be an eigenvalue of the matrix $\hat{C}_{\mathrm{rb}}\left(\omega, k_{x}, k_{y}\right)$, and let $\mu=\hat{\jmath}\left(k_{x}, k_{y}\right)$. Then, $\lambda$ and $\mu$ are related via the quadratic equation

$$
\lambda^{2}-2\left(1-\omega+\frac{\omega^{2} \mu^{2}}{2}\right) \lambda+(1-\omega)^{2}=0 .
$$

Note that as $\omega$ varies, the eigenvalues $\lambda_{1}$ and $\lambda_{2}$ move about the complex plane. We are interested in how the quantity $\rho=\max \left(\left|\lambda_{1}\right|,\left|\lambda_{2}\right|\right)$ depends on $\omega$. When viewed as a function of $\omega$, the discriminant

$$
\Delta=4(1-\omega) \omega^{2} \mu^{2}+\omega^{4} \mu^{4}
$$

of (5.19) has a real root at

$$
\omega_{d}=\frac{2}{1+\sqrt{1-\mu^{2}}} .
$$

It is easy to check that $\rho<1$ if and only if $0<\omega<2$. Furthermore, we have

$$
\rho= \begin{cases}\frac{1}{4}\left[\omega|\mu|+\sqrt{\left.\omega^{2} \mu^{2}+4(1-\omega)\right]^{2}},\right. & 0 \leq \omega \leq \omega_{d} \\ \omega-1 & \omega_{d} \leq \omega \leq 2 .\end{cases}
$$

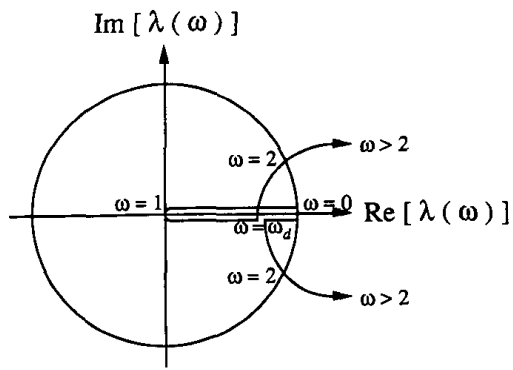

Fig. 5. Root loci of $\lambda_{1}$ and $\lambda_{2}$ with fixed $\mu$.

The locus of eigenvalues $\lambda_{1}$ and $\lambda_{2}$ as $\omega$ varies is plotted in Fig. 5. When $\omega=0$, the eigenvalues $\lambda_{1}$ and $\lambda_{2}$ coincide at the value 1 . As $\omega$ increases from 0 to 1 , both eigenvalues move toward the origin along the real line but with different speeds. When $\omega$ reaches 1 , the eigenvalues are 0 and $\mu^{2}$. When $1<\omega \leq \omega_{d}$, one eigenvalue increases its value from 0 and the other continues to decrease. They coincide again at the point $\omega_{d}-1$ when $\omega=\omega_{d}$. The eigenvalues become complex conjugate pair with magnitude $\omega-1$ for $\omega>\omega_{d}$. Thus, these eigenvalues lie outside of the unit circle for $\omega$ $>2$. This plot shows that the spectral radius $\rho$ is minimized for $\omega=\omega_{\mathrm{d}}$.

Since $\mu=\hat{\jmath}\left(k_{x}, k_{y}\right)$, the relaxation parameter $\omega_{d}$ which minimizes the spectral radius of $\hat{G}_{\mathrm{rb}}\left(\omega, k_{x}, k_{y}\right)$ is a function of the wavenumber $\left(k_{x}, k_{y}\right)$. In order to minimize the spectral radius of the space-domain operator $G_{\mathrm{rb}}(\omega)$, we must therefore select for $\omega$ the value which minimizes the maximum over all feasible wavenumbers of the spectral radius of $\hat{G}\left(\omega, k_{x}\right.$ $k_{y}$ ). A straightforward analysis [75] shows that the optimal relaxation parameter $\omega_{\text {opt }}$ is given by the value of $\omega_{d}$ corresponding to the wavenumber $\left(k_{x}, k_{y}\right)=(1,1)$. Since $\hat{j}(1$, 1) $=\cos (\pi h)$, we obtain

$$
\omega_{\mathrm{opt}}=\frac{2}{1+\sqrt{1-\cos ^{2}(\pi h)}} \approx 2-2 \pi h
$$

and the corresponding spectral radius is

$$
\rho\left(G_{\mathrm{rb}}\left(\omega_{\mathrm{opt}}\right)\right)=\omega_{\mathrm{opt}}-1 \approx 1-2 \pi h .
$$

We see from (5.22) that the number of iterations required by the red-black SOR iteration to reduce the error by a constant factor is $\mathrm{O}\left(h^{-1}\right)$, so that this algorithm is one order of magnitude faster than the Jacobi or red-black Gauss-Seide relaxations. However, this rate of convergence is achieved only when the relaxation paramater is equal to its optimal value $\omega_{\text {opt }}$, and is sensitive to perturbations of the relaxation parameter away from this value.

An interesting feature of the SOR method is that, since the optimum relaxation parameter $\omega_{\text {opt }}$ is larger than $\omega_{d}$ for all wavenumber components $\left(k_{x}, k_{y}\right) \neq(1,1)$, the eigenvalues of $G_{\mathrm{rb}}\left(\omega_{\mathrm{opt}}\right)$ have all the same magnitude $\omega_{\mathrm{opt}}-1$. To illustrate this phenomenon, the spectra of the Jacobi and SOR (with $\omega_{\text {opt }}$ ) iteration matrices are plotted in Fig. 6 . The eigenvalue of the Jacobi iteration matrix are all real and occur in + - pairs. Their magnitude ranges from 0 to cos $(\pi h)=1-O\left(h^{2}\right)$. Thus, different Fourier components converge at different rates, and the slowest converging Fourier component is the one that establishes the convergence rate of the Jacobi method. Through the SOR acceleration, these eigenvalues are redistributed around a circle of radius $\omega_{\mathrm{opt}}$ 


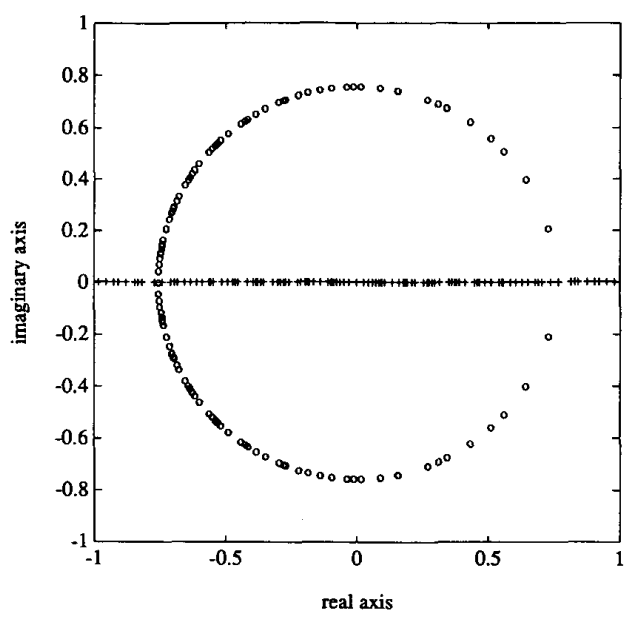

Fig. 6. A typical eigenvalue map in the complex plane for the Jacobi iteration $(+)$ and the SOR iteration $(0)$ with the optimal relaxation parameter, where the case $h=\frac{1}{16}$ and $\omega$ $=1.757$ is plotted.

$-1=1-O(h)$ in the complex plane. Since they have the same magnitude, all Fourier components converge at the same rate. Thus the acceleration effect of the SOR method is achieved by balancing the convergence rates of the different Fourier components.

\section{Polynomial Acceleration}

The SOR procedure is a stationary one-step acceleration technique, in the sense that it optimizes the convergence behavior of one iteration, and uses the same acceleration scheme at every subsequent iteration. There exists an alternative acceleration approach which optimizes the convergence behavior of the overall algorithm, instead of considering only one step. Specifically, if a given iterative procedure requires $s$ steps to converge, we can select a set of acceleration parameters $\omega_{i}$ with $1 \leq i \leq s$ and apply $\omega_{i}$ at the $i$ th iteration to increase the convergence rate. This approach leads to the polynomial acceleration method described below.

Consider the sequence of iterates generated by the iteration

$$
W^{(m+1)}=P W^{(m)}+c
$$

where $P$ is assumed to have real eigenvalues, and $\rho(P)<$ 1 , so that (5.23) converges. For example, one possible choice for $P$ is the Jacobi iteration matrix $J$. The error $e_{w}^{(m)}=w^{(m)}-$ $\bar{w}$ at the $m$ th iteration is given by

$$
e_{w}^{(m)}=P^{m} e_{w}^{(0)} \text {. }
$$

To improve the convergence of the sequence $\left\{w^{(m)}\right\}$, we can generate a new sequence $\left\{u^{(m)}\right\}$ by performing a linear combination

$$
u^{(m)}=\sum_{i=0}^{m} \alpha_{m, i} w^{(i)}
$$

where the coefficients $\alpha_{m, i}$ are real and satisfy

$$
\sum_{i=0}^{m} \alpha_{m, i}=1
$$

for all $m$. This condition is imposed in order to guarantee that when $w^{(0)}=\bar{u}$, then $u^{(m)}=\bar{u}$ for $m \geq 0$. Let $e^{(m)}$ be the error associated with the new sequence $u^{(m)}$. From (5.24) and (5.25), we can relate $e^{(m)}$ and $e_{w}^{(m)}$ via

$$
\mathrm{e}^{(m)}=Q_{m}(P) e_{w}^{(0)}, \quad \text { where } Q_{m}(P)=\sum_{i=0}^{m} \alpha_{m, i} P^{m}
$$

is a matrix polynomial of degree $m$. Since $e^{(0)}=e_{w}^{(0)}$, the errors associated with the $\left\{u^{(m)}\right\}$ iteration satisfy

$$
e^{(m)}=Q_{m}(P) e^{(0)} \text {. }
$$

The problem is to select the coefficients $\alpha_{m, i}$ so that the error sequence $\left\{\mathrm{e}^{(m)}\right\}$ converges to zero as fast as possible.

Since $Q_{m}(P)$ is a polynomial function of $P$, it has the same eigenvectors as $P$, and if $\mu$ is an eigenvalue of $P$, the eigenvalue of $Q_{m}(P)$ corresponding to the same eigenvector is $Q_{m}(\mu)$. Let $S$ be the discrete spectrum of the matrix $P$, and let $\mu_{\min }$ and $\mu_{\max }$ denote the smallest and largest eigenvalues of $P$. The polynomial acceleration problem can be formulated as the minimax problem

$$
\min _{\alpha_{m, i}} \max _{x \in S}\left|Q_{m}(x)\right|
$$

Since the discrete spectrum $S$ is seldom known, the problem (5.28) cannot usually be solved as such. A modified version which is easier to solve consists in replacing $S$ in (5.28) by the continuous spectrum $\bar{S}=\left\{x: \mu_{\min } \leq x \leq \mu_{\max }\right\}$. In this case, we can perform the change of variable

$$
z(x)=\frac{2 x-\left(\mu_{\max }+\mu_{\min }\right)}{\mu_{\max }-\mu_{\min }}
$$

so that (5.28) is transformed into a minimax problem defined on the interval $[-1,1]$. The solution of this new minimax problem is well known and is given by the Chebyshev polynomial of order $m, T_{m}(z)$. In terms of the original variable $x$, the solution is

$$
Q_{m}(x)=T_{m}(z(x)) / T_{m}(z(1))
$$

where the scaling by $T_{m}(z(1))$ ensures that the coefficient constraint (5.26) is satisfied.

An interesting property of Chebyshev polynomials is that they satisfy the three-term recurrence relation

$$
T_{m+1}(z)=2 z T_{m}(z)-T_{m-1}(z), \quad m \geq 1
$$

with $T_{0}(z)=1$ and $T_{1}(z)=z$. This property can be exploited to generate the new sequence $\left\{u^{(m)}\right\}$ efficiently, instead of using expression (5.25), which has a high computational cost, and requires a large amount of storage. By taking into account the recursions (5.23) and (5.31) inside (5.25), we obtain the following Chebyshev semi-iterative (CSI) acceleration procedure [57], [96] for iteration (5.23):

$$
\begin{aligned}
u^{(m+1)}= & \beta_{m+1}\left[\gamma\left(P u^{(m)}+c\right)+(1-\gamma) u^{(m)}\right] \\
& +\left(1-\beta_{m+1}\right) u^{(m-1)}
\end{aligned}
$$

with

$$
\begin{aligned}
& \gamma=\frac{2}{2-\mu_{\max }-\mu_{\min }} \\
& \beta_{1}=1, \quad \beta_{2}=\left(1-\frac{1}{2} \sigma^{2}\right)^{-1}, \\
& \quad \beta_{m+1}=\left(1-\frac{1}{4} \sigma^{2} \beta_{m}\right)^{-1}, \quad m \geq 2
\end{aligned}
$$


and

$$
\sigma=\frac{\mu_{\max }-\mu_{\min }}{2-\mu_{\max }-\mu_{\min }} .
$$

To illustrate the redistribution of the eigenvalues of $P$ which is accomplished by the $\mathrm{CSI}$ acceleration method, the function $Q_{10}(x)$ describing how the eigenvalues of $Q_{m}(P)$ depend on those of $P$ for $m=10$ is plotted in Fig. 7. From

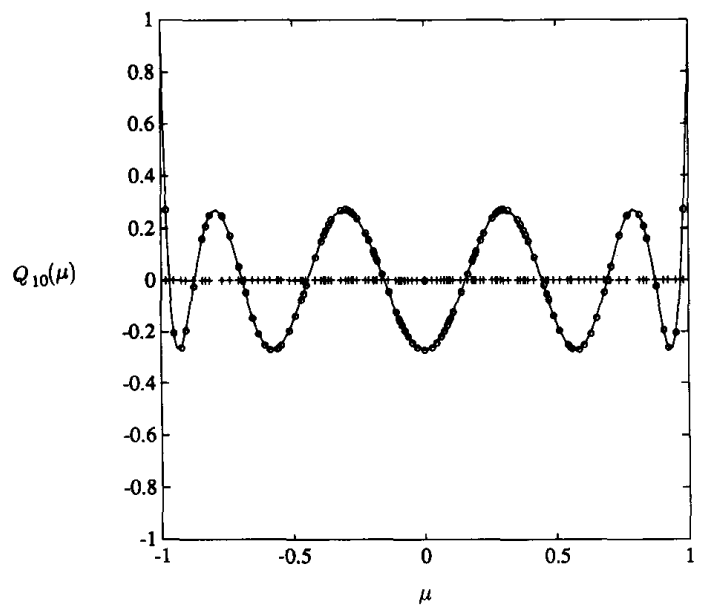

Fig. 7. Typical eigenvalue distribution for the Chebyshev semi-iterative method plotted as function of the eigenvalues of the lacobi iteration $\left(h=\frac{1}{16}, \mu_{\max }=-\mu_{\min }=0.98\right.$ and $m$ $=10$ ).

this figure, we see that unlike the SOR method, where the eigenvalues of $G_{r b}\left(\omega_{o p t}\right)$ were all complex and equal in magnitude, the eigenvalues of the CSI matrix $Q_{m}(P)$ remain real, and lie in the narrow interval

$$
\left[-2 r^{m / 2} /\left(1+r^{m}\right), 2 r^{m / 2} /\left(1+r^{m}\right)\right]
$$

with

$$
r=\frac{1-\sqrt{1-\sigma^{2}}}{1+\sqrt{1-\sigma^{2}}}
$$

As an example, consider the case where the CSI method is used to accelerate the Jacobi iteration for the model Poisson problem with Dirichlet conditions, so that $P=J$ in (5.23). The resulting algorithm is called the J-CSI method. The asymptotic convergence rate of the J-CSI method can be determined as follows. From (5.8), we know that

$$
\mu_{\max }=\cos (\pi h), \quad \mu_{\min }=-\cos (\pi h)
$$

and from (5.33c),

$$
\sigma=\cos (\pi h) .
$$

Then, observing from Fig. 7 that the maximum value of $\left|Q_{m}(x)\right|$ over the interval $\left[\mu_{\min }, \mu_{\max }\right]$ is reached for $x=\mu_{\max }$ we find that

$$
\rho\left(Q_{m}(J)\right)=\left|Q_{m}\left(\mu_{\max }\right)\right|=2 \frac{r^{m / 2}}{1+r^{m}}
$$

where, from (5.34),

$$
r=\frac{1-\sin (\pi h)}{1+\sin (\pi h)}
$$

According to (5.27), the error of the J-CSI method at the $m$ th iteration is obtained by multiplying the initial error by $\mathrm{Q}_{m}(J)$. The asymptotic error contraction factor per iteration is therefore

$$
\lim _{m \rightarrow \infty}\left(\rho\left(Q_{m}(J)\right)^{1 / m}\right)=r^{1 / 2} \approx 1-\pi h .
$$

This shows that the J-CSI method requires $O\left(h^{-1}\right)$ iterations to reduce the error by a constant factor. A further improvement in this algorithm was introduced by Golub and Varga [51], who observed that for the the red-black ordering, the recursion (5.32) can be rearranged in such a way that only the odd iterates of the red points and the even iterates of the black points need to be computed, thus cutting the numerical complexity of the algorithm in half. The resulting procedure is called the cyclic CSI method, and its numerical complexity is the same as that of the SOR method.

\section{Historical Notes}

The development of relaxation methods for the solution of large systems of linear equations was initiated by Gauss, Jacobi, and Seidel in the 19th century, and Ridchardson, Liebmann, and Southwell early in this century. Since a comprehensive account of the history of relaxation methods can be found in a recent paper by Young [103], our comments focus primarily on the application of Fourier analysis to the study of these methods. The development of the SOR theory in the late 1940s [42], [100], [101] marked the beginning of a period of rapid progress in the area of iterative methods. The Fourier approach adopted in this section has for origin the work of Frankel [42] and Young [100], who used Fourier-like basis functions to analyze the SOR method applied to the naturally ordered Poisson problem with Dirichlet boundary conditions. Recently, LeVeque and Trefethen [77] reinterpreted Frankel's result from a tilted grid viewpoint. The same problem with periodic boundary conditions was analyzed by Chan and Elman [24]. The two-color Fourier analysis of the SOR method for the red-black ordered model Poisson problem with Dirichlet or periodic boundary conditions was developed by Kuo et al. [70], [75]. The use of Chebyshev polynomials was first proposed by Flanders and Shortley [41] for the solution of matrix eigenvalue problems, and subsequently led to the development of the Chebyshev semi-iterative (CSI) method for solving linear systems. A complete discussion of elementary relaxation methods and of the SOR and CSI acceleration procedures can be found in books by Birkhoff and Lynch [14], Hageman and Young [57], Varga [96], and Young [102].

\section{Multigrid Methods}

The major limitation of elementary and accelerated relaxation methods is that while the components of the error decrease very rapidly in certain frequency bands, they decay only very slowly in other bands. The region of rapid decay depends on the specific relaxation method that we consider, but it consists typically of middle or high frequencies. On the other hand, the region of slow decay always includes the low frequencies. This phenomenon reflects the fact that the low frequency components of the solution depend on global information, and a large number of iterations are required for propagating information from the edges of the problem domain to its center. Since the error becomes pro- 
gressively smoother as the iteration proceeds, it is natural to consider switching to a coarser discretization grid, where we can assume temporarily that an exact solver is available. This solver can be used to compute the smooth components of the error on the coarse grid, and the resulting correction can then be interpolated back to the fine grid and combined with the original fine grid solution. Such a solution scheme is called a two-grid method. In this approach the fine grid provides the accuracy required by the approximation while the coarse grid offers a faster convergence rate for the low frequency Fourier components. Naturally, the weakness of the above scheme is that we have assumed that an exact solver is available on the coarse grid. This is generally an unreasonable assumption, but we need only to observe that the problem on the coarse grid can itself be solved by a two-grid method. By proceeding recursively, we obtain a multigrid scheme, where progressively coarser grids are employed, until so few discretization points are involved that a direct solver can be used to compute the error on the coarsest grid. The resulting solution technique is called a multigrid method.

Since the two-grid method is the main component of multigrid methods, our first step in this section is to perform a detailed analysis of the two-grid iteration operator. We use two-color Fourier analysis to find the spectrum of this operator for the 1-D and 2-D model Poisson problems. Then, we describe several of the standard recursion patterns, namely the V-cycle, W-cycle, and full-multigrid schemes, that are used to generate multigrid methods from the twogrid iteration.

\section{A. Two-Grid Iteration}

Consider two discretization grids $\Omega_{h}$ and $\Omega_{2 h}$, with mesh sizes $h$ and $2 h$, respectively, and let

$$
L_{h} u_{h}=f_{h}
$$

be the equation that we seek to solve on the fine grid, where $L_{h}, f_{h}$ and $u_{h}$ denote the discretized operator, forcing function, and solution, respectively. An $(h, 2 h)$ two-grid iteration for solving this equation consists of the following three steps.

Step 1: Presmoothing: Select a relaxation operator $S_{h}$ for solving (6.1) on the fine grid. Typically, $S_{h}$ is the Gauss-Seidel relaxation, but other choices are possible, such as the damped Jacobi iteration described below. Then, given an initial estimate $u_{h}^{(0)}$ of the solution, apply the $S_{h}$ iteration $\nu_{1}$ times. If $u_{h}^{(1)}$ denotes the resulting approximate solution, the corresponding residual is

$$
r_{h}=f_{h}-L_{h} u_{h}^{(1)} \text {. }
$$

Step 2: Coarse-Grid Correction: The residual $r_{h}$ can be projected onto the coarse grid $\Omega_{2 h}$ by using a restriction operator $I_{h}^{2 h}$, thus yielding $f_{2 h}=I_{h}^{2 h} r_{h}$. Then, since we assume that an exact solver is available on $\Omega_{2 h}$, we use this solver, which is denoted here by $L_{2 h}^{-1}$, to find the solution $u_{2 h}$ of the coarse grid problem

$$
L_{2 h} u_{2 h}=f_{2 h} \text {. }
$$

If $I_{2 h}^{h}$ denotes an interpolation operator for transferring a function defined on $\Omega_{2 h}$ onto the fine grid $\Omega_{h}$, we can interpolate the coarse grid correction $u_{2 h}$, and add it to the solution obtained in Step 1, thus yielding

$$
u_{h}^{(2)}=u_{h}^{(1)}+I_{2 h}^{h} u_{2 h} \text {. }
$$

Step 3: Postsmoothing: Using $u_{h}^{(2)}$ as initial solution, we apply the $S_{h}$ iteration $\nu_{2}$ times. The resulting approximate solution is $u_{h}^{(3)}$

The above three steps are illustrated in Fig. 8. Usually, the numbers $\nu_{1}$ and $\nu_{2}$ of pre- and post-smoothing iterations are 0,1 or 2 , and $\nu=\nu_{1}+\nu_{2}$ is 2 or 3 . If

$$
e^{\text {old }}=u_{h}^{\text {old }}-\bar{u}, \quad e^{\text {new }}=u_{h}^{\text {new }}-\bar{u}
$$

are the solution errors before and after one full two-grid iteration, the error dynamics for the two-grid iteration can be expressed as

$$
e^{\text {new }}=M_{h}^{2 h} e^{\text {old }}
$$

where the two-grid iteration operator $M_{h}^{2 h}$ is given by

$$
M_{h}^{2 h}=S_{h}^{\nu_{2}} K_{h}^{2 h} S_{h}^{\nu_{1}}
$$

and $K_{h}^{2 h}$ is the coarse grid correction operator

$$
K_{h}^{2 h}=I_{h}-I_{2 h}^{h} L_{2 h}^{-1} I_{h}^{2 h} L_{h} \text {. }
$$

Naturally, the two-grid iteration needs to be repeated until the error becomes sufficiently small. It will be shown below ihat the two-grid iteration operator $M_{h}^{2 h}$ reduces the error by a constant factor independent of $h$, so that only $O\left(\log \left(h^{-1}\right)\right)$ iterations are necessary to solve (6.1) within the discretization accuracy $O\left(h^{P}\right)$, where $p$ is a positive integer.

Note that equations (6.2)-(6.6) provide only a general description of the two-grid iteration procedure. In order to obtain an actual two-grid iteration, we need to select the operators $S_{h}, I_{h}^{2 h}, I_{2 h}^{h}$, and $L_{2 h}$ which have been left unspecified in the above description. In spite of the fact that there exist many different ways to choose these operators and

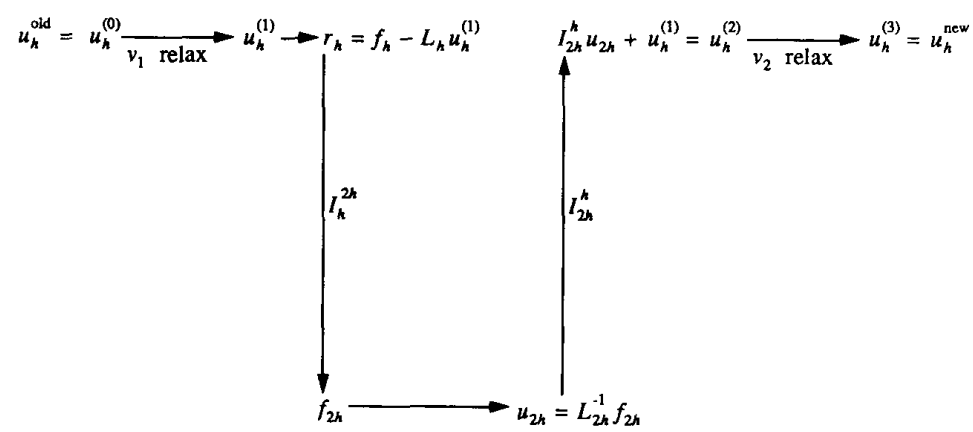

Fig. 8. Structure of an $(h, 2 h)$ two-grid method. 
that they need to be adjusted to achieve the best convergence performance for different applications, the efficiency of multigrid methods does not usually depend on this choice. It is the utilization of multiple discretization grids that makes these methods converge very rapidly. In the following subsections, $S_{h}$ is the red-black Gauss-Seidel iteration operator, $L_{2 h}$ is the usual 3-point (resp. 5-point) discretization of the 1-D (resp. 2-D) Poisson operator on the grid $\Omega_{2 h}$, and $I_{h}^{2 h}$ and $I_{2 h}^{h}$ are the full weighting restriction and linear interpolation operators, respectively.

\section{B. Solution of the 1-D Poisson Problem}

Two-Grid Method and Analysis: Consider an $(h, 2 h)$ twogrid method for solving the discretized 1-D Poisson equation

$$
\frac{1}{h^{2}}\left(u_{n-1}-2 u_{n}+u_{n+1}\right)=f_{n}, \quad 1 \leq n \leq N-1
$$

where the boundary values $u_{0}$ and $u_{N}$ are given, $h$ is the grid spacing, and $N=h^{-1}$ is even. For the 1-D problem (6.8), it will be shown below that it is possible to choose the relaxation, restriction and interpolation operators so that $M_{h}^{2 h}$ $=0$. This means that the two-grid method is a direct solver for (6.8). However, this is not true in general for 2-D or 3-D problems.

Quite often, a simple but crude technique, called the smoothing rate analysis [18], can be used to study the convergence behavior of two-grid or multigrid methods. This analysis assumes that the coarse-grid correction operator $K_{h}^{2 h}$ annihilates all the low frequency components of the error and preserves its high frequency components, i.e.,

$$
\hat{K}_{h}^{2 h}(k)= \begin{cases}0, & 1 \leq k<N / 2 \\ 1, & N / 2 \leq k \leq N-1 .\end{cases}
$$

By expressing (6.6) in the frequency domain and using assumption (6.9), we find that the two-grid iteration operator admits the frequency domain representation

$$
\hat{M}_{h}^{2 h}(k)= \begin{cases}0, & 1 \leq k<N / 2 \\ \hat{S}_{h}^{\nu_{1}+v_{2}}(k), & N / 2 \leq k \leq N-1,\end{cases}
$$

where $\hat{S}_{h}(k)$ denotes the spectrum of $S_{h}$. The largest magnitude $\mu$ of $\hat{S}_{h}(k)$ for $N / 2 \leq k \leq N-1$ is called the smoothing factor. Therefore, the convergence rate of the two-grid method is related to the smoothing factor via

$$
\rho\left(M_{h}^{2 h}\right)=\mu^{\nu_{1}+\nu_{2}} .
$$

To give an example, consider the damped Jacobi iteration,

$$
u_{n}^{(m+1)}=(1-\omega) u_{n}^{(m)}+\frac{\omega}{2}\left(u_{n+1}^{(m)}+u_{n-1}^{(m)}-h^{2} f_{n}\right)
$$

where $\omega$ is a relaxation parameter. The damped Jacobi smoother has the spectrum

$$
\hat{\jmath}(\omega, k)=(1-\omega)+\omega \cos (k \pi h)
$$

whose magnitude parameterized with $\omega$ is plotted in Fig. 9. We can choose $\omega$ to minimize the magnitude of the largest eigenvalue in the high frequency region. The optimal relaxation parameter is $\omega=2 / 3$, which is obtained by solving

$$
\hat{\jmath}\left(\omega, \frac{N}{2}\right)=-\hat{\jmath}(\omega, N)
$$

and the corresponding smoothing rate is

$$
\mu=\max _{N / 2 \leq k \leq N-1}\left|\hat{\jmath}\left(\frac{2}{3}, k\right)\right|=\frac{1}{3} .
$$

The estimated two-grid convergence rate becomes

$$
\rho\left(M_{h}^{2 h}\right)=\left(\frac{1}{3}\right)^{\eta_{1}+\nu_{2}} .
$$

We should point out that the assumption (6.9) for the smoothing rate analysis does not actually hold in practice. However, because of its simplicity, this analysis is often useful for estimating the convergence behavior of multigrid methods.

There are situations where the smoothing rate analysis predicts completely wrong results. One such case arises when the red-black Gauss-Seidel relaxation is used as smoother. Following a procedure similar to the one employed for deriving (5.11), we find that with respect to the coefficients $\left(\hat{e}_{r, k}, \hat{e}_{b, k}\right)$ of the 1-D red-black Fourier series

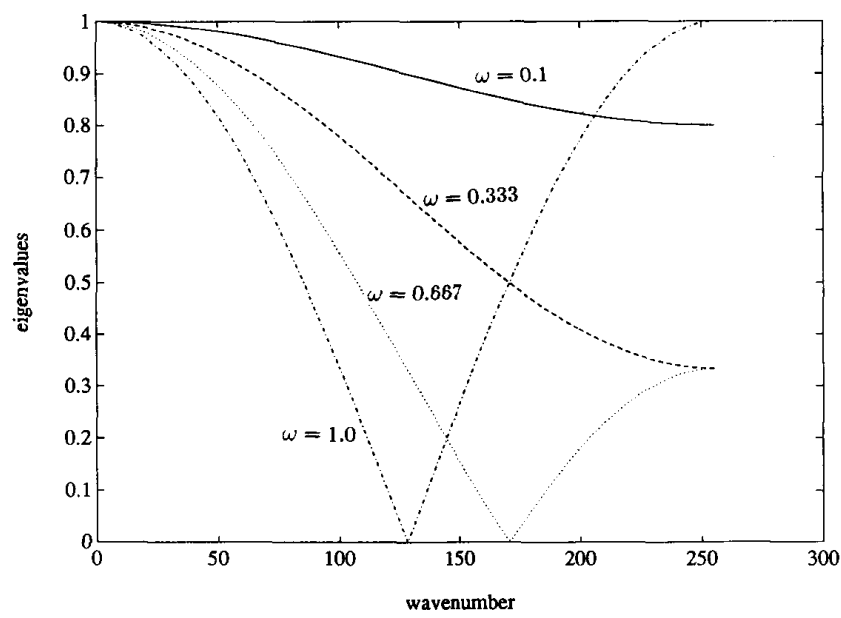

Fig. 9. The spectrum of the 1-D damped Jacobi smoother parameterized with $\omega(N=256)$. 
expansion

$$
\begin{array}{ll}
e_{n} & =\sum_{k=1}^{N / 2-1} \hat{e}_{r, k} \sin (k \pi n h), \quad n \text { even } \\
e_{n} & =\sum_{k=1}^{N / 2} \hat{e}_{b, k} \sin (k \pi n h), \quad n \text { odd }
\end{array}
$$

the red-black Gauss-Seidel relaxation operator $G_{r b}$ can be represented as

$$
\hat{\mathrm{C}}_{\mathrm{rb}}(k)=\left[\begin{array}{cc}
0 & \cos (k \pi h) \\
0 & \cos ^{2}(k \pi h)
\end{array}\right], \quad 1 \leq k \leq N / 2-1
$$

with $\hat{G}_{\mathrm{rb}}(N / 2)=0$. The expression (6.18) holds also for high frequency components $(k>N / 2)$ which are aliased into the low frequency region. Thus, the red-black Gauss-Seidel smoother attenuates rapidly the middle frequency components $(k \approx N / 2)$ but works poorly for the low and high frequencies. According to the smoothing rate analysis, we have

$$
\max _{N / 2 \leq k \leq N-1}\left|\hat{G}_{\mathrm{rb}}(k)\right|=\cos ^{2}(\pi h) \approx 1-\pi^{2} h^{2} .
$$

This implies a poor convergence of the correspoding multigrid method. However, contrary to this prediction, numerical experiments show that the multigrid method with the red-black Gauss-Seidel smoother is an exact solver for the 1-D Poisson problem and converges very rapidly in the 2-D case. Thus, in order to explain the effectiveness of the red-black Gauss-Seidel smoother, we cannot assume that the condition (6.9) holds. It is necessary to perform a complete two-grid analysis, i.e., to study the spectrum of the coarse-grid corrector $K_{h}^{2 h}$ defined in (6.7), as well as that of the smoother $S_{h}$.

We have first to define more precisely the operators appearing in (6.2)-(6.4). The $h$-grid and $2 h$-grid Laplacians are

$$
L_{h}=\frac{1}{h^{2}}\left(E_{h}^{-1}-2+E_{h}\right) ; L_{2 h}=\frac{1}{(2 h)^{2}}\left(E_{2 h}^{-1}-2+E_{2 h}\right)
$$

where $E_{2 h}=E_{h}^{2}$. To restrict a function from $\Omega_{h}$ to $\Omega_{2 h}$, we perform an averaging operation with coefficients $1 / 4,1 / 2$ and $1 / 4$ and then down-sample the averaged sequence on $\Omega_{2 h}$. The restriction operator is denoted by

$$
I_{h}^{2 h}:\left|\frac{1}{4}, \frac{1}{2}, \frac{1}{4}\right|_{h}^{2 h} \text {. }
$$

To interpolate a function from $\Omega_{2 h}$ to $\Omega_{h}$, we use a linear interpolation scheme for grid points belonging to $\Omega_{h}-\Omega_{2 h}$. The interpolation operator is written as

$$
I_{2 h}^{h}:\left|\frac{1}{2}, 1, \frac{1}{2}\right|_{2 h}^{h} .
$$

With respect to the red-black Fourier expansion (6.17), the action of the $h$-grid discretized Laplacian and identity operator $I_{h}$ on the red-black Fourier vector $\left(\hat{e}_{r, k}, \hat{e}_{b, k}\right)^{T}$ can be represented by the $2 \times 2$ matrices

$$
\hat{L}_{h}(k)=\frac{2}{h^{2}}\left[\begin{array}{cc}
-1 & \cos (k \pi h) \\
\cos (k \pi h) & -1
\end{array}\right] ; \hat{l}_{h}(k)=\left[\begin{array}{ll}
1 & 0 \\
0 & 1
\end{array}\right] .
$$

Observing that in the 1-D case, the points of the coarse grid coincide with the red points of the fine grid, we find that the red-black spectral representations of the restriction and interpolation operators $I_{h}^{2 h}$ and $I_{2 h}^{h}$ correspond respectively to mappings from $\left(\hat{e}_{r, k}, \hat{e}_{b, k}\right)^{T}$ onto $\hat{e}_{r, k}$ and from $\hat{e}_{r, k}$ onto $\left(\hat{e}_{r, k}, \hat{e}_{b, k}\right)^{T}$, and are given by

$$
\hat{I}_{h}^{2 h}(k)=\left[\frac{1}{2}, \frac{\cos (k \pi h)}{2}\right] ; i_{2 h}^{h}(k)=\left[\begin{array}{c}
1 \\
\cos (k \pi h)
\end{array}\right] .
$$

Furthermore, with respect to the Fourier component $\hat{e}_{r, k}$ the $2 h$-grid discretized Laplacian is represented by the spectrum

$$
\hat{L}_{2 h}(k)=\frac{2(\cos (2 k \pi h)-1}{(2 h)^{2}} .
$$

We obtain therefore

$$
\begin{aligned}
\hat{K}_{h}^{2 h}(k) & =\hat{l}_{h}(k)-\hat{I}_{2 h}^{h}(k) \hat{L}_{2 h}^{-1}(k) \hat{l}_{h}^{2 h}(k) \hat{L}_{h}(k) \\
& =\left[\begin{array}{cc}
0 & 0 \\
-\cos (k \pi h) & 1
\end{array}\right] .
\end{aligned}
$$

Finally, choosing $S_{h}=G_{\mathrm{rb}}$ and $\nu_{1}=\nu_{2}=1$ in (6.6), we find that the red-black spectral representation of the two-grid operator is given by

$$
\hat{M}_{h}^{2 h}(k)=\hat{G}_{\mathrm{rb}}(k) \hat{K}_{h}^{2 h}(k) \hat{G}_{\mathrm{rb}}(k) .
$$

From (6.18) and (6.23), it is easy to check that $\hat{M}_{h}^{2 h}(k)$ are 2 $\times 2$ zero matrices for $1 \leq k \leq N / 2-1$ and $\hat{M}_{h}^{2 h}(N / 2)=0$. Thus, the two-grid method with red-black Gauss-Seidel smoothing is a direct solver.

Multigrid methods: The implementation of the two-grid method requires inverting the coarse-grid Laplacian operator $L_{2 h}$. An efficient way to carry out this inversion is to use a $(2 h, 4 h)$ two-grid iteration. By using nested two-grid iterations, we can therefore reduce the original problem to one defined on progressively coarser grids, until a direct solver can be used to invert the discretized operator on the coarsest grid. Thus, if the mesh-size on the finest grid is $h=2^{-L}$ with $L>2$, the following nested iteration specifies an $L$-grid solver:

$$
M_{h}^{2 h}=G_{\mathrm{rb}}\left(I_{h}-I_{2 h}^{h} X_{2 h} I_{h}^{2 h}\right) G_{\mathrm{rb}}
$$

with

$$
X_{h}= \begin{cases}M_{h}^{2 h}, & \text { for } h=2^{-1}, 2 \leq l \leq L-1 \\ L_{h}^{-1}, & \text { for } h=1 / 2 .\end{cases}
$$

One can prove by induction that this multigrid algorithm solves the 1-D Poisson problem directly. It is possible to simplify this algorithm to save computations. See [74] for details.

\section{Solution of the 2-D Poisson Problem}

Let $L_{h}$ and $L_{2 h}$ be the 5-point discretizations of the Laplacian on $\Omega_{h}$ and $\Omega_{2 h}$, i.e.,

$$
\begin{aligned}
& L_{h}=\frac{1}{h^{2}}\left(E_{h, x}+E_{h, x}^{-1}-4+E_{h, y}+E_{h, y}^{-1}\right) \\
& L_{2 h}=\frac{1}{(2 h)^{2}}\left(E_{2 h, x}+E_{2 h, x}^{-1}-4+E_{2 h, y}+E_{2 h, y}^{-1}\right) .
\end{aligned}
$$


Then, $I_{h}^{2 h}$ and $I_{2 h}^{h}$ denote the full-weighting restriction and linear interpolation operators, given respectively by

$$
I_{h}^{2 h}:\left|\begin{array}{ccc}
\frac{1}{16} & \frac{1}{8} & \frac{1}{16} \\
\frac{1}{8} & \frac{1}{4} & \frac{1}{8} \\
\frac{1}{16} & \frac{1}{8} & \frac{1}{16}
\end{array}\right|_{h}^{2 h}
$$

and

$$
I_{2 h}^{h}:\left|\begin{array}{ccc}
\frac{1}{4} & \frac{1}{2} & \frac{1}{4} \\
\frac{1}{2} & 1 & \frac{1}{2} \\
\frac{1}{4} & \frac{1}{2} & \frac{1}{4}
\end{array}\right|_{2 h}^{h} .
$$

We consider only the case $1 \leq k_{x}, k_{y}<N / 2$. Each of the $4 \times 4$ frequency domain matrices appearing below corresponds to a mapping from the vector space spanned by

$$
\left(\hat{r}_{\mathrm{k}},-\hat{r}_{\mathrm{k}}, \hat{b}_{\mathrm{k},}-\hat{b}_{\mathrm{k}}\right)^{T}
$$

onto itself, where

$$
\begin{aligned}
& \mathbf{k}=\left(k_{x}, k_{y}\right), \quad 1 \leq k_{x}, k_{y}<\frac{N}{2} ; \\
& \mathbf{k}= \begin{cases}\left(N-k_{x}, k_{y}\right), & \text { for } k_{x} \geq k_{y} \\
\left(k_{x}, N-k_{y}\right), & \text { for } k_{x}<k_{y} .\end{cases}
\end{aligned}
$$

When $k_{x}$ or $k_{y}$ is equal to $N / 2$, the $4 \times 4$ matrices reduce to $2 \times 2$ or $1 \times 1$ matrices. The analysis of these degenerate cases can be found in [74] and is omitted here. We also use the abbreviations

$$
\begin{aligned}
& \alpha=\frac{\cos \theta_{x}+\cos \theta_{y}}{2} ; \bar{\alpha}=\frac{\cos \bar{\theta}_{x}+\cos \tilde{\theta}_{y}}{2} ; \\
& \beta=\cos \theta_{x} \theta_{y} ; \bar{\beta}=\cos \bar{\theta}_{x} \cos \bar{\theta}_{y}
\end{aligned}
$$

where $\theta_{x}=k_{x} \pi h, \theta_{y}=k_{y} \pi h, \tilde{\theta}_{x}=\tilde{k}_{x} \pi h$, and $\bar{\theta}_{y}=\tilde{k}_{y} \pi h$.

The matrices representing operators $I_{h}, L_{h}$ and $L_{2 h}^{-1}$ in the frequency domain can be written as

$$
\begin{aligned}
\hat{l}_{h}\left(k_{x}, k_{y}\right) & =\left[\begin{array}{ll}
l & 0 \\
0 & I
\end{array}\right] ; \hat{L}_{h}\left(k_{x}, k_{y}\right)=\frac{4}{h^{2}}\left[\begin{array}{rr}
-I & I \\
J & -1
\end{array}\right] \\
\hat{L}_{2 h}^{-1}\left(k_{x}, k_{y}\right) & =\frac{h^{2}}{2 \delta^{\prime}}, \delta \equiv 2 \alpha^{2}-\beta-1
\end{aligned}
$$

where 0 is the $2 \times 2$ zero matrix, $l$ is the $2 \times 2$ identity matrix, and

$$
J=\operatorname{diag}(\alpha, \tilde{\alpha}) .
$$

The decomposition shown in Fig. 10, which is commonly used in multirate digital signal processing [31], provides a

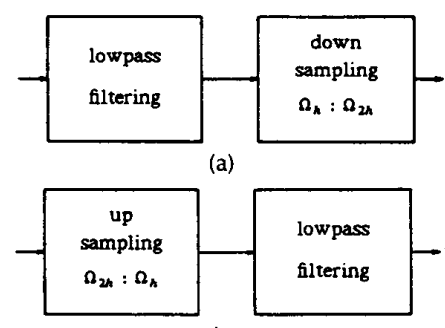

(b)

Fig. 10. Decomposition of the (a) restriction and (b) interpolation operators.

KUO AND LEVY: DISCRETIZATION AND SOLUTION OF ELLIPTIC PDES simple physical interpretation of the interpolation and restriction operators, and is also useful for deriving their frequency domain matrices. In this decomposition, the restriction procedure $I_{h}^{2 h}$ is divided into two steps,

Step 1: Lowpass filtering (or averaging) at every point of $\Omega_{h}$, where the weighting coefficients are specified by the stencil (6.27a).

Step 2: Down-sampling (or injecting) values from $\Omega_{h}$ to $\Omega_{2 h}$.

The interpolation operator $I_{2 h}^{h}$ is also decomposed into two steps,

Step 1: Up-sampling values from $\Omega_{2 h}$ to $\Omega_{h}$, where we assign 0 to points which belong to $\Omega_{h}-\Omega_{2 h}$.

Step 2: Lowpass filtering at every point of $\Omega_{h}$, where the weighting coefficients are specified by the stencil $(6.27 \mathrm{~b})$.

It is relatively easy to find a frequency domain matrix representation for each of the above steps. Combining them together, we obtain

$$
\hat{l}_{h}^{2 h}\left(\theta_{x}, \theta_{y}\right)=\left[\begin{array}{llll}
1 & 1 & 0 & 0
\end{array}\right]
$$

$$
\begin{aligned}
\times \frac{1}{4}\left[\begin{array}{llll}
1+\beta & 0 & 2 \alpha & 0 \\
0 & 1+\tilde{\beta} & 0 & 2 \bar{\alpha} \\
2 \alpha & 0 & 1+\beta & 0 \\
0 & 2 \tilde{\alpha} & 0 & 1+\bar{\beta}
\end{array}\right] \\
=\frac{1}{4}\left[\begin{array}{llll}
1+\beta & 1+\tilde{\beta} & 2 \alpha & 2 \tilde{\alpha}
\end{array}\right]
\end{aligned}
$$

and

$$
\begin{aligned}
\hat{l}_{2 h}^{h}\left(\theta_{x}, \theta_{y}\right) & =\left[\begin{array}{llll}
1+\beta & 0 & 2 \alpha & 0 \\
0 & 1+\tilde{\beta} & 0 & 2 \tilde{\alpha} \\
2 \alpha & 0 & 1+\beta & 0 \\
0 & 2 \tilde{\alpha} & 0 & 1+\bar{\beta}
\end{array}\right] \times \frac{1}{2}\left[\begin{array}{l}
1 \\
1 \\
0 \\
0
\end{array}\right] \\
& =\frac{1}{2}\left[\begin{array}{l}
1+\beta \\
1+\bar{\beta} \\
2 \alpha \\
2 \bar{\alpha}
\end{array}\right] .
\end{aligned}
$$

Thus, in the frequency domain, the down-sampling operation adds the high frequency component $-\hat{r}_{k}$ to the low frequency component $\hat{~}_{k}$. This phenomenon is known as aliasing [31]. Similarly, the up-sampling operation sets the high-frequency component $-\hat{r}_{\bar{k}}$ equal to the low-frequency component $\hat{r}_{k}$. This duplication effect is called imaging [31]. The lowpass filters which are cascaded with the down-and up-sampling operations reduce the aliasing and imaging effects. For example, when $\theta_{x}$ and $\theta_{y}$ are close to $0, \alpha \approx 1$, $\beta \approx 1, \tilde{\alpha} \approx 0$, and $\tilde{\beta} \approx-1$. Hence, the aliasing and imaging effects occurring between $\left(\hat{r}_{k}, \hat{b}_{k}\right)^{T}$ and $\left(\hat{r}_{k}, \hat{b}_{\vec{k}}\right)^{T}$ are substantially eliminated by the associated lowpass filters.

From (6.30) and (6.31), we can compute the spectrum $\hat{K}_{h}^{2 h}\left(k_{x}, k_{y}\right)$ of the coarse-grid correction operator. The frequency domain matrix corresponding to the red-black Gauss-Seidel iteration is

$$
\hat{\mathrm{G}}_{\mathrm{rb}}\left(k_{x}, k_{y}\right)=\left[\begin{array}{ll}
0 & j \\
0 & J^{2}
\end{array}\right] .
$$


Note that $\hat{G}_{r b}\left(k_{x}, k_{y}\right)$ is a matrix of rank 2 rather than 4 . Combining the spectra of the smoothing and coarse-grid correction operators, we obtain the spectrum of the two-grid operator

$$
\hat{M}_{h}^{2 h}\left(k_{x}, k_{y}\right)=\hat{G}_{\mathrm{rb}}^{\nu_{1}}\left(k_{x}, k_{y}\right) \hat{K}_{h}^{2 h}\left(k_{x}, k_{y}\right) \hat{G}_{\mathrm{rb}}^{\nu_{2}}\left(k_{x}, k_{y}\right)
$$

which is again a matrix of rank 2. In [92] this feature was exploited to find a closed-form expression for the spectral radius of the two-grid operator. If $\nu=\nu_{1}+\nu_{2}$, we get

$$
\rho\left(M_{h}^{2 h}\right)= \begin{cases}\frac{1}{4^{\prime}} & \nu=1 \\ \frac{1}{2 \nu}\left(\frac{\nu}{\nu+1}\right)^{\nu+1}, & \nu \geq 2 .\end{cases}
$$

In (6.34), the maximum of $\rho\left[\hat{M}_{h}^{2 h}(\theta)\right]$ occurs at $\theta=(\pi / 2,0)$ or $(0, \pi / 2)$ when $\nu=1$ and at $\left(\cos ^{-1}\left[(\nu / \nu+1)^{1 / 2}\right], \cos ^{-1}[(\nu / \nu+\right.$ 1) ${ }^{1 / 2}$ ]) when $\nu \geq 2$. Note since $M_{h}^{2 h} \neq 0$, the two grid method is not a direct solver in the 2-D case. However, the spectral radius $\rho$ is a constant independent of the grid size $h$, so that only $O\left(\log \left(h^{-1}\right)\right)$ two-grid iterations are needed to solve (6.1) with an accuracy equal to the 5-point discretization error $O\left(h^{2}\right)$.

Multigrid methods: As in the 1-D case, we can recursively invoke the two-grid method to obtain multigrid algorithms. However, different recursion patterns may be needed for different 2-D or 3-D problems. Three commonly used recursion patterns, the $\mathrm{V}$-cycle and $\mathrm{W}$-cycle and full multigrid algorithms are shown in Fig. 11.

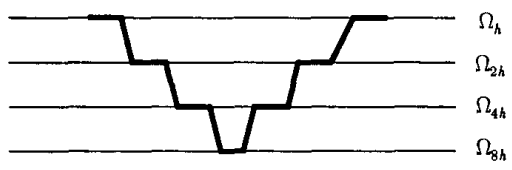

(a)

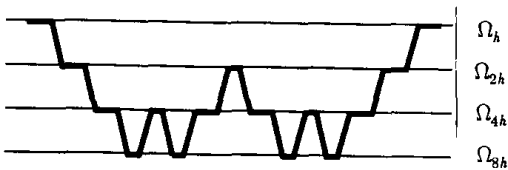

(b)

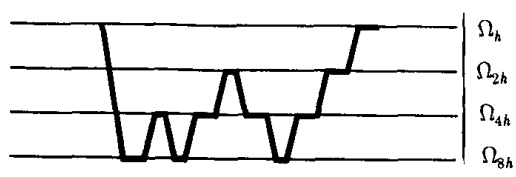

(c)

Fig. 11. Illustration of (a) V-cycle, (b) W-cycle, and (c) full multigrid methods.

From this figure, we see that while the $V$-cycle multigrid algorithm applies the coarse-grid correction operator once per cycle, the W-cycle algorithm applies it twice. The nummerical complexity per cycle of the $\mathrm{V}$-cycle algorithm is therefore smaller than that of the W-cycle algorithm. On the other hand, since the $W$-cycle algorithm yields a better approximation of $L_{2 h}^{-1}$, it requires fewer cycles to converge. The choice of cycling scheme depends on how the above tradeoff is affected by the problem that we seek to solve.
For the model Poisson problem, the $\mathrm{V}$-cycle algorithm works well. It requires just a few cycles (two or three) to converge within a fixed accuracy (independent of $h$ ), so that there is no need to use the W-cycle algorithm. However, the W-cycle algorithm is usually superior for difficult problems, such as highly anisotropic or nonlinear problems.

In the full multi-grid (FMG) scheme, instead of solving the discretized problem (6.1) on the fine grid only, we solve it on all grids, starting from the coarsest grid. Once (6.1) has been solved within the discretization accuracy of a given grid, we interpolate the solution to the next finer grid, and use this solution as initial estimate for the $\mathrm{V}$-or W-cycle multigrid algorithm applied to the next problem. The advantage of this approach is that, because we are using a good initial estimate for each successive problem, only a constant number of $\mathrm{V}$ - or $\mathrm{W}$-cycle iterations are needed to solve (6.1) within the discretization error $O\left(h^{P}\right)$ of each grid. The total computational cost of the FMG algorithm is therefore very small, and equals the cost of a constant number of smoothing iterations on the finest grid [18], [54], [92].

\section{Historical Notes}

The idea of solving elliptic PDEs by using relaxation on multiple grids was first proposed by Fedorenko [39] and Bakhvalov [12] in the 1960s. However, it was not until the work of Brandt [18], Nicolaides [83], and Hackbush [54] in the 1970s that the efficiency of multigrid methods are recognized, and that their convergence properties were fully analyzed. Brandt used Fourier analysis to study the errorsmoothing rate in the high frequency region. Subsequently, Stüben and Trottenberg [92] also used a Fourier approach to analyze a complete two-grid method including fine-grid smoothing, restriction, coarse-grid inversion and interpolation. Since all the elements of multigrid methods are already present in a two-grid cycling scheme, the results obtained for this scheme are usually a good indicator of the performance of more general multigrid algorithms. More recently, it was shown in [74] that the analysis of two-grid iterations can be simplified significantly by using two-color Fourier analysis. The book by Briggs [20] and article by Jespersen [63] provide a good introduction to multigrid methods for readers not acquainted with the subject. The proceedings of European multigrid conferences in 1981 [55] and 1985 [56] include several interesting theoretical and practical contributions, particularly concerning the application of multigrid methods to problems of fluid dynamics and aerodynamics. A book edited recently by McCormick [81] contains several articles on various aspects of multigrid theory, as well as an exhaustive multigrid bibliography until 1987. Finally, [54] gives a rigorous mathematical treatment of multigrid methods, and in particular of their convergence properties.

\section{Preconditioned Conjugate Gradient Methods}

In the previous two sections, we have examined relaxation methods for solving elliptic PDEs on single and multiple grids. In this section, we consider solution techniques which combine the conjugate gradient algorithm with a preconditioning procedure, whose role is to reduce the condition number of the original system, thereby decreasing accordingly the number of iterations required by the conjugate gradient algorithm. 


\section{A. The Preconditioned Conjugate Gradient (PCG)} Algorithm

When the conjugate gradient (CG) algorithm was introduced in the 1950s to solve SPD (symmetric positive definite) systems of the form (3.1), it was considered by some researchers as a direct method, since in the absence of roundoff errors, it yields an exact solution in at most $N$ steps, where $N$ is the order of the system. However, because of roundoff errors, this finite termination property does not hold in practice. Furthermore, since the SOR or CSI methods require only $O\left(N^{1 / 2} \log N\right)$ iterations for the model Poisson problem, the conjugate gradient algorithm would in fact be relatively inefficient if it truly required $N$ steps to solve this problem.

This forced researchers to view the CG method as an iterative method, and in this context it was found that a useful bound for the norm of the error $e^{(m)}$ after $m$ iterations is [7], [8]

$$
\left\|\mathrm{e}^{(m)}\right\|_{A} \leq\left\|\mathrm{e}^{(0)}\right\|_{A}\left[\frac{\sqrt{\kappa}-1}{\sqrt{\kappa}+1}\right]^{m}
$$

where $\kappa(A)$ denotes the condition number of the matrix $A$ in (3.1), and $\|x\|_{A}=\left(x^{T} A x\right)^{1 / 2}$. For the 2D model Poisson problem, since for Dirichlet or periodic boundary conditions the eigenvalues are given respectively by $(3.14)$ or (3.17), it is easy to check that $\kappa(A)=O\left(h^{-2}\right)=O(N)$. Substituting this value inside the bound (7.1) we can conclude that the CG procedure reduces the error by a constant factor in at most $O\left(N^{1 / 2}\right)$ iterations, so that its rate of convergence is comparable to that of the SOR and CSI methods.

Although the bound (7.1) is rather conservative since it does not take into account the clustering of the eigenvalues of $A$, it provides an important clue for improving the CG method. Specifically, by introducing a SPD preconditioning transformation $M$, the system (3.1) can be transformed into

$$
\tilde{A} \tilde{u}_{d}=\tilde{f}_{d}
$$

where $\bar{A}, \tilde{u}_{d}$ and $\tilde{f}_{d}$ are related to $A, u_{d}$ and $f_{d}$ via

$$
\tilde{A}=M^{1 / 2} A M^{-1 / 2}, \quad \tilde{u}_{d}=M^{1 / 2} u_{d r} \quad \tilde{f}_{d}=M^{1 / 2} f_{d}
$$

and $M^{1 / 2}$ denotes the symmetric square-root of $M$. From the definition of $\tilde{A}$, we see that it is SPD. If the transformation $M$ is easy to invert, and if the condition number $\kappa(\tilde{A})$ of the transformed system is much less than $\kappa(A)$, it becomes advantageous to apply the CG algorithm to the preconditioned system (7.2) instead of the original system (3.1). Note that since the matrices $\tilde{A}$ and $M^{-1} A$ are related by a similarity transform, we can examine the spectrum of $M^{-1} A$ instead of that of $\tilde{A}$ in order to find the convergence rate of the PCG method. In the following, $M$ and $M^{-1} A$ will be called respectively the preconditioner and the preconditioner operator.

With the initialization:

$$
x_{0} \text { arbitrary, } \quad r_{0}=p_{0}=b-A x_{0}, \quad \text { and } \beta_{1}=0
$$

the $k+1$ th $(k=0,1,2, \cdots)$ iteration of the PCG algorithm consists of the following two steps [50]:

Step 1: Preconditioning: Solve

$$
M z_{k}=r_{k}
$$

for $z_{k}$.
Step 2: CG iteration: Compute

$$
\begin{aligned}
& \beta_{k+1}=\left(z_{k}, r_{k}\right) /\left(z_{k-1}, r_{k-1}\right) \\
& p_{k+1}=z_{k}+\beta_{k+1} p_{k} \\
& \alpha_{k+1}=\left(z_{k}, r_{k}\right) /\left(p_{k+1}, A p_{k+1}\right) \\
& x_{k+1}=x_{k}+\alpha_{k+1} p_{k+1} \\
& r_{k+1}=r_{k}-\alpha_{k+1} A p_{k+1} .
\end{aligned}
$$

If the spectrum of $\bar{A}$ has no special clustering feature, and if the condition number $k(\tilde{A}) \gg 1$, the bound $(7.1)$ for the error norm indicates that the number of PCG iterations required to reduce the error by a constant factor is proportional to $O(\sqrt{\kappa(\hat{A}))}$. Thus, the goal of preconditioning is to find preconditioners $M$ which are easy to invert, since each PCG iteration requires the solution of a system of the form (7.4), and such that the condition number of $\tilde{A}$ is as small as possible.

If both $A$ and $M$ have Fourier functions as eigenfunctions, the spectrum of $M^{-1} A$ can be analyzed directly in the frequency domain. In this context, the design of preconditioners corresponds to an inverse filtering problem. That is, given an FIR filter $A$, we seek to construct a filter $M^{-1} \approx$ $A^{-1}$ such that $M^{-1}$ can be implemented efficiently. Note that since $A^{-1}$ is a noncausal IIR filter, this last constraint precludes selecting $M=A$.

Many elliptic preconditioners have been proposed in the literature. Depending on whether they rely on operations performed on a single discretization grid, or a sequence of discretization grids, they fall into the category of singlelevel, or of multi-level preconditioners. Examples of singlelevel preconditioners include the SSOR (symmetric successive over-relaxation) [5], ILU (incomplete lower and upper factorization) [82], MILU (modified ILU) [36] methods, as well as polynomial preconditioners [4], [64]. Examples of multilevel preconditioners include the multigrid method [66], [67] as well as the $\mathrm{HB}$ (hierarchical basis) [104], [84], and MF (multilevel filtering) [71], [95] preconditioners. Since the design of elliptic preconditioners is an active research area, we do not attempt to survey all existing preconditioning techniques. Instead, our goal is to relate the design and analysis of some preconditioners to familar concepts in DSP to motivate further research along this line.

\section{B. Preconditioners Based on Incomplete Factorization}

Among single-level preconditioners, we focus on those obtained by incomplete factorization. Note that the Cholesky algorithm can be used to factor the coefficient matrix $A$ into a product of lower and upper triangular matrices. However, although $A$ is sparse, the Cholesky algorithm results in fillin for its lower and upper triangular factors. The amount of fillin depends on the bandwidth $B$ of $A$, which may be significant, say $O\left(N^{1 / 2}\right)$ for a discretized self-adjoint elliptic PDE problem. The resulting band Cholesky algorithm then requires $O\left(N B^{2}\right)$ operations [50], p. 155. We are therefore led to consider preconditioners which require only an approximate factorization of $A$, i.e., $A \approx L U$, and with a computational complexity of $O(N)$. Efficient approximate factorization procedures of this type can be obtained by requiring that the lower and upper triangular factors $L$ and $U$ should have the same sparsity pattern as $A$. From the multidimensional signal processing viewpoint, construct- 
ing an incomplete factorization is equivalent to factoring of a noncausal IIR filter $A^{-1}$ approximately into the product of two causal IIR filters $U^{-1}$ and $L^{-1}$ of fixed size.

The ILU and MILU factorizations, which were originally introduced in [82] and [36] respectively, rely on two different rules for constructing $L$ and $U$. Both factorizations require that $L$ and $U$ should have the same zero entries as the lower and upper triangular parts of $A$, and that the nonzero offdiagonal entries of $A$ should be equal to the corresponding entries of $M=L U$. The difference between the two factorizations lies in the way the diagonal elements of $M$ are specified (see Fig. 12). For the ILU factorization, the diag-
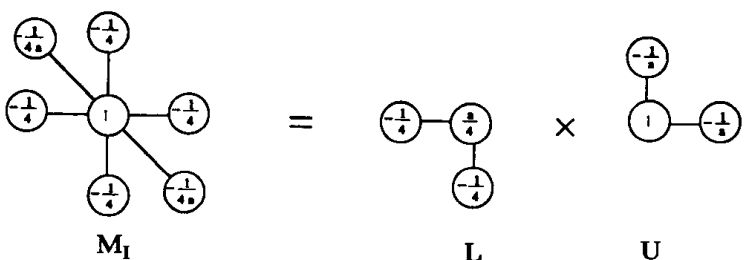

(a)

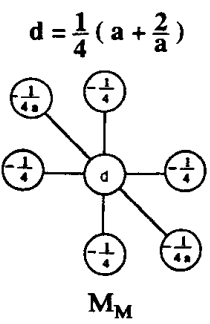

$=$

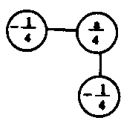

$\times$

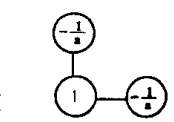

(b)

$$
U\left(E_{x}, E_{y}\right)=1-\frac{1}{a} E_{x}-\frac{1}{a} E_{y}
$$

where $a$ is a constant to be determined. Since the only nonzero coefficients of $L\left(E_{x}, E_{y}\right)$ (resp. $U\left(E_{x}, E_{y}\right)$ ) are those of 1 , $E_{x}^{-1}$ and $E_{y}^{-1}$ (resp. $1, E_{x}$ and $\left.E_{y}\right), L$ and $U$ have the same sparsity pattern as the lower and upper triangular parts of $A\left(E_{x \prime}\right.$ $\left.E_{y}\right)$. The local ILU preconditioners $M_{i}\left(E_{x}, E_{y}\right)$ is the product of $L\left(E_{x}, E_{y}\right)$ and $U\left(E_{x}, E_{y}\right)$ :

$$
\begin{aligned}
M_{i}\left(E_{x}, E_{y}\right)= & \frac{1}{4}\left[a+\frac{2}{a}-\left(E_{x}+E_{y}+E_{x}^{-1}+E_{y}^{-1}\right)\right. \\
& \left.+\frac{1}{a}\left(E_{x} E_{y}^{-1}+E_{x}^{-1} E_{y}\right)\right] .
\end{aligned}
$$

Comparing (3.7) and (7.7), we see that the coefficients of the off-diagonal terms $E_{x}, E_{x}^{-1}, E_{y}$, and $E_{y}^{-1}$ of operator $A\left(E_{x}, E_{y}\right)$ are matched by those of $M_{l}\left(E_{x}, E_{y}\right)$. Note that $M_{l}$ contains some additional off-diagonal terms of the form $E_{x} E_{y}^{-1}$ and $E_{x}^{-1} E_{y}$. The ILU factorization imposes the additional requirement that the coefficients of the diagonal terms of $M_{I}$ and $A$ should be the same. This implies (see Fig. 12)

$$
a+\frac{2}{a}=4
$$

so that $a=2+\sqrt{2}$. This value of $a$ is in fact observed asymptotically in the ILU factorization of the model Poisson problem with Dirichlet boundary conditions.

Therefore, the ILU-preconditioned Laplacian can be written in operator form as

$$
\begin{aligned}
\left(M_{l}^{-1} A\right)\left(E_{x}, E_{y}\right)= & {\left[1-\frac{1}{4}\left(E_{x}+E_{y}+E_{x}^{-1}+E_{y}^{-1}\right)\right.} \\
& \left.+\frac{1}{8+4 \sqrt{2}}\left(E_{x}+E_{y}^{-1}+E_{x}^{-1} E_{y}\right)\right]^{-1} \\
& \times\left[1-\frac{1}{4}\left(E_{x}+E_{y}+E_{x}^{-1}+E_{y}^{-1}\right)\right] .
\end{aligned}
$$

It is straightforward to compute the spectrum of $M_{l}^{-1} A$ with respect to the Fourier basis functions $\mathrm{e}^{i 2 \pi\left(k_{x} n_{x}+k_{y} n_{y}\right) h}$. We obtain

row sum of $A$ by a small quantity $c h^{2}$, where $\mathrm{c}$ is a constant independent of $h$.

$$
\hat{M}_{l}^{-1}\left(k_{x}, k_{y}\right) \hat{A}\left(k_{x}, k_{y}\right)=\frac{1-\frac{1}{2}\left[\cos \left(k_{x} 2 \pi h\right)+\cos \left(k_{y} 2 \pi h\right)\right]}{\left.1-\frac{1}{2}\left[\cos \left(k_{x} 2 \pi h\right)+\cos \left(k_{y} 2 \pi h\right)\right]+\frac{1}{4+2 \sqrt{2}} \cos \left(k_{x}-k_{y}\right) 2 \pi h\right)}
$$

Each row of the matrix factors $L$ and $U$ specifies local finitedifference operators $L\left(E_{x}, E_{y}\right)$ and $U\left(E_{x}, E_{y}\right)$. Even if the PDE discretization operator $A\left(E_{x}, E_{y}\right)$ has constant coefficients the local operators $L\left(E_{x}, E_{y}\right)$ and $U\left(E_{x}, E_{y}\right)$ have usually spacedependent coefficients, due to boundary effects. However, for points far away from the domain boundary, these coefficients tend asymptotically to constant values. In the following, we ignore boundary effects and restrict our attention to the asymptotic behavior of incomplete factorization preconditioners.

ILU Preconditioners: For the model Poisson problem with the natural ordering, the local factorization operators $L\left(E_{x}\right.$ $\left.E_{y}\right)$ and $U\left(E_{x}, E_{y}\right)$ take the form [82]

$$
L\left(E_{x}, E_{y}\right)=\frac{1}{4}\left(a-E_{x}^{-1}-E_{y}^{-1}\right)
$$

where $k_{x}$ and $k_{y}$ are integers between 1 and $N-1$. This spectrum is plotted in Fig. 13. From this plot, as well as from a direct analysis, it is easy to check that the spectrum reaches its minimum at the four corners of the domain $1 \leq k_{x}, k_{y}$ $\leq N-1$, and its maximum at the center, i.e., for $k_{x}=k_{y}$ $\approx N / 2$. Furthermore, the minimum and maximum are proportional to $O\left(h^{2}\right)$ and $O(1)$, respectively. This gives

$$
\kappa_{l}(\tilde{A})=\frac{\lambda_{\max }(\tilde{A})}{\lambda_{\min }(\tilde{A})}=\frac{\lambda_{\max }\left(M_{l}^{-1} A\right)}{\lambda_{\min }\left(M_{l}^{-1} A\right)}=O\left(h^{-2}\right) .
$$

Since the condition number of $\tilde{A}$ is of the same order as that of $A$, it is tempting to conclude that the ILU factorization is not a good preconditioner for the CG algorithm. However, from Fig. 13, we see that except at the four corners of the $\left(k_{x}, k_{y}\right)$ domain, the eigenvalues of $\tilde{A}$ are close to 1 . A consequence of this eigenvalue clustering property is that 


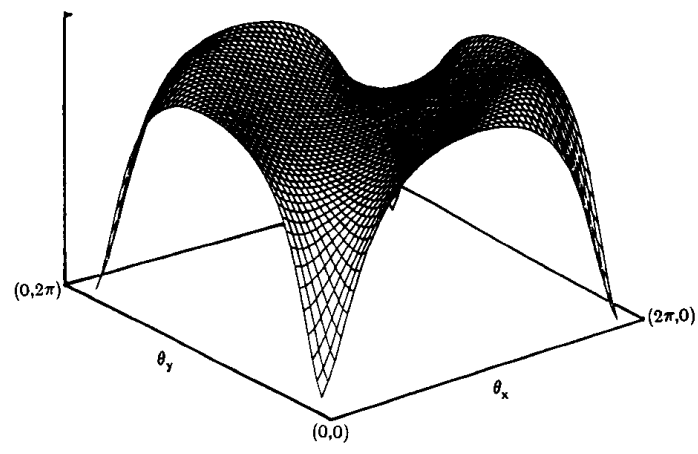

Fig. 13. Typical surface plot of the spectrum of the ILU preconditioned Laplacian where $\theta_{x}=2 \pi k_{x} h, \theta_{y}=2 \pi k_{y} h$ and $h=0.02$.

the ILU preconditioner has a significant acceleration effect on the CG algorithm which is not reflected by the bound (7.1).

MILU Preconditioner: The MILU preconditioner has the same sparsity pattern as the ILU preconditioner, so that (7.6) and (7.7) also apply. Thus, for the model Poisson problem with the natural ordering, the MILU preconditioner can be represented as

$$
\begin{aligned}
M_{M}\left(E_{x}, E_{y}\right)= & \frac{1}{4}\left[a+\frac{2}{a}-\left(E_{x}+E_{y}+E_{x}^{-1}+E_{y}^{-1}\right)\right. \\
& \left.+\frac{1}{a}\left(E_{x} E_{y}^{-1}+E_{x}^{-1} E_{y}\right)\right] .
\end{aligned}
$$

The difference between the ILU and MILU factorizations lies in how the constant $a$ is determined. For the MILU factorization [36], it is required that the row sum of $M_{M}\left(E_{x}, E_{y}\right)$ should differ from the row sum $A\left(E_{x}, E_{y}\right)$, which is zero, by a small quantity $\delta$. This gives

$$
\frac{1}{4}\left(a+\frac{4}{a}-4\right)=\delta
$$

and selecting $\delta=c h^{2} / 4$ with c $>0$, we obtain

$$
a=2+\frac{c h^{2}}{2}+\frac{1}{2} \sqrt{8 c h^{2}+c^{2} h^{4}} .
$$

As was observed above, the spectrum of the ILU preconditioner $M_{1}$ approximates poorly the spectrum of $A$ at the four corners of the domain $1 \leq k_{x}, k_{y} \leq N-1$. In the modified ILU scheme, the condition (7.13) is imposed in order to guarantee that the preconditioner $M_{M}$ approximates $A$ well in this region. By performing a Fourier analysis identical to the one employed for the ILU case, the spectrum and condition number of the MILU-preconditioned Laplacian can be evaluated. A surface plot of the spectrum is shown in Fig. 14. This plot indicates that the smallest eigenvalues are of order 1 , and the largest eigenvalues occur near the end points of the transverse diagonal $k_{x}+k_{y}=N$. These eigenvalues are of order $h^{-1}$, and consequently

$$
\kappa_{M}(\tilde{A})=\frac{\lambda_{\max }\left(M_{M}^{-1} A\right)}{\lambda_{\min }\left(M_{M}^{-1} A\right)}=O\left(h^{-1}\right) .
$$

Comparing (7.11) and (7.15), we see that the condition number of the MILU preconditioned system is one order of magnitude smaller than that of the ILU preconditioned system.

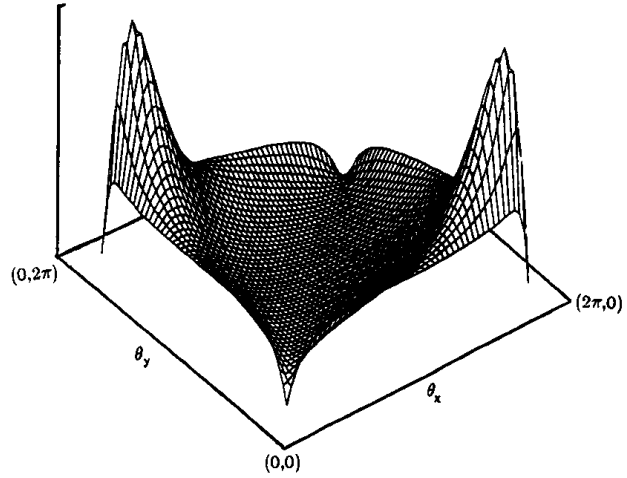

Fig. 14. Typical surface plot of the spectrum of the MILU preconditioned Laplacian where $\theta_{x}=2 \pi k_{x} h, \theta_{y}=2 \pi k_{y} h, h$ $=0.02$ and $c=70$.

Numerical experiments have confirmed that the ILU-CG and MILU-CG require, respectively, $O\left(h^{-1}\right)$ and $O\left(h^{-1 / 2}\right)$ iterations to converge [24].

The ordering of grid points plays in general an important role in determining the form of the coefficient matrix $A$, and hence of the preconditioners. With the red-black ordering, the ILU and MILU preconditioners take completely different forms and the spectra of preconditioned operators behave very differently. See [70] for more details.

\section{Multilevel Preconditioners Based on Filtering}

The focus of research on elliptic preconditioners has shifted recently to the design of preconditioners with a multilevel (or hierarchical) grid structure. Since the global features of elliptic operators can be reproduced more easily by multilevel preconditioners, the resulting preconditioned systems have often very small condition numbers, ranging from $O(1)$ to $O\left(\log ^{\alpha} h^{-1}\right)$ where $\alpha$ is a small integer, and hence the corresponding PCG algorithms converge very quickly. Another advantage of multilevel preconditioners is that they can be effectively implemented on massively parallel computers [71] and, therefore, are attractive for parallel computation.

Several multilevel preconditioners have been proposed. One such preconditioner is the MG algorithm of Section VI. When combined with the CG method, it yields the MG$\mathrm{CG}$ algorithm. The motivation for using the $\mathrm{MG}$ algorithm as a preconditioner is that its speed of convergence is governed by the smoothness of the solution function, whereas the convergence rate of the CG method is not affected by this feature. Consequently, the MG-CG method is more effective than the MG method alone for certain applications, such as the solution of interface problems, where because of presence of several materials, the elliptic PDE has discontinuous coefficients. Two other types of multilevel preconditioners have been proposed by Yserentant [104], [105] and Bramble, Pasciak, and Xu [17], [99] in the context of finite-element methods. Yserentant considered a new set of basis functions, known as the hierarchical basis. Bramble et al. introduced a sequence of basis functions which are defined at various discretization levels and called multilevel nodal basis functions. Roughly speaking, the preconditioning step $M^{-1} r$ consists in projecting the residual $r$ onto these basis functions. In the following, we exam- 
ine yet another preconditioner, the multilevel filtering (MF) preconditioner, which was proposed recently in [71]. This preconditioner relies explicitly on multirate digital signal processing techniques and can be best described in the Fourier domain.

The filtering approach to the design of preconditioners can be described as follows. Suppose that we approximate the spectrum of an elliptic operator by a piecewise-constant function. In the space domain, this approximating function corresponds to an operator which (i) splits the input function into several components, where each such component consists of wavenumbers within a narrow band, (ii) scales each component by a constant, and (iii) recombines all the scaled components. The inverse of such an operator is easy to implement, since it has the same form, except that the scaling constants are inverted. In multirate digital signal processing, the decomposition of a signal into components consisting of different wavenumber bands, and vice versa, is accomplished by a filter bank analyzer (resp. synthesizer). Although there exists a number of techniques for designing filter banks (see [31], Chapter 7), the filter bank which is used for the MF preconditioning technique is obtained by cascading a sequence of lowpass operating on different discretization grids, in combination with down- and up-sampling operations.

To be more precise, consider the 1D Poisson equation on $[0,1]$ with zero boundary conditions. After discretization on a uniform grid $\Omega_{h}$ with spacing $h=2^{-L}$, where $L$ is a positive integer, we obtain

$$
\left(-\frac{1}{2} E+1-\frac{1}{2} E^{-1}\right) u_{n}=f_{n}, \quad 1 \leq n \leq N-1
$$

with $N=2^{L}$. This system can be rewritten as

$$
A u=f
$$

where $A$ is the tridiagonal matrix with diagonal elements $-1 / 2,1$ and $-1 / 2$. $A$ can be diagonalized as

$$
A=W^{\top} \Lambda_{A} W
$$

where

$$
\begin{aligned}
\Lambda_{A} & =\operatorname{diag}\left(\lambda_{1}, \cdots, \lambda_{k}, \cdots, \lambda_{N-1}\right), \\
\lambda_{k} & =1-\cos (k \pi h)
\end{aligned}
$$

and $W$ is a square matrix of size $N-1$, whose $k$ th row is

$$
\begin{gathered}
w_{k}^{T}=\left(\frac{2}{N}\right)^{1 / 2}(\sin (k \pi h), \cdots, \sin (k \pi n h), \cdots, \\
\quad \sin (k \pi(N-1) h)) .
\end{gathered}
$$

The diagonalization of the matrix $A$ can be interpreted as a decomposition of the driving and solution functions into their Fourier components. Furthermore, $\lambda_{k}$ is just the spectrum $\hat{A}(k)$ of the 1D Laplacian.

In the wavenumber domain, the spectrum $\hat{A}(k)$ can be approximated by a piecewise constant function

$$
\hat{P}(k)=c_{l,} \quad k \in B_{l}, \quad 1 \leq l \leq L
$$

where

$$
B_{l}=\left\{k \in N: 2^{\prime}-1 \leq k<2^{\prime}\right\}
$$

denotes the /th wavenumber band. Let $\Lambda_{p}$ be the diagonal matrix with $\hat{P}(k)$ as $k$ th diagonal element and $P=W^{T} \Lambda_{P} W$.
Then, the P-preconditioned Laplacian takes the form

$$
P^{-1} A=W^{T} \Lambda_{P-1} A W
$$

with

$$
\begin{array}{r}
\Lambda_{P-1 A}=\left(\Lambda_{P}\right)^{-1} \Lambda_{A}=\operatorname{diag}\left(\frac{\lambda_{1}}{c_{1}}, \frac{\lambda_{2}}{c_{2}}, \frac{\lambda_{3}}{c_{2}}, \cdots\right. \\
\left.\frac{\lambda_{2^{\prime-1}}}{c_{1}}, \cdots, \frac{\lambda_{2^{\prime}-1}}{c_{1}}, \cdots, \frac{\lambda_{N-1}}{c_{L}}\right) .
\end{array}
$$

The question is how to choose the constants $c_{l}$ in order to reduce the condition number of $P^{-1} A$. If we select

$$
c_{l}=4^{-(L-1)}
$$

it can be shown [71] that the eigenvalues of $P^{-1} A$ satisfy

$$
1 \leq \lambda\left(P^{-1} A\right)<\frac{\pi^{2}}{2} \approx 4.93
$$

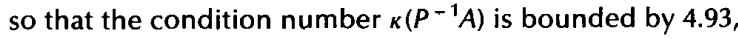
a constant independent of the grid size $h$. In Figure 15, we

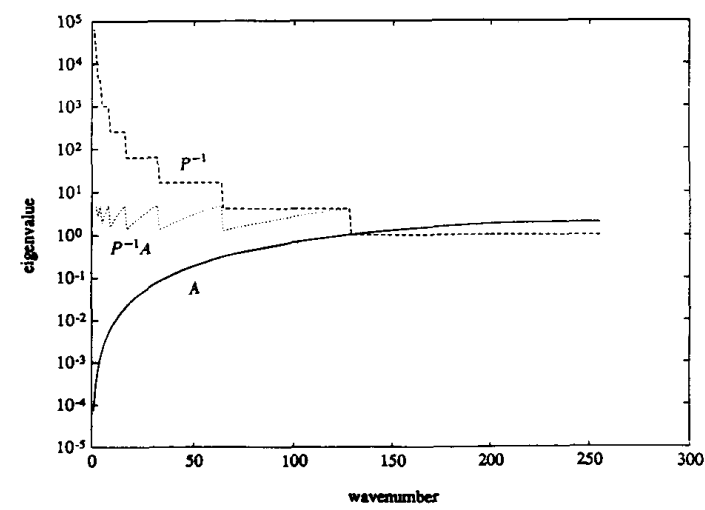

Fig. 15. Spectra of $A, P^{-1}$ and $P^{-1} A$

plot the spectra $\hat{A}(k), \hat{P}^{-1}(k)$ and $\hat{P}^{-1}(k) A(k)$ for $N=h^{-1}=$ 256, when $c_{i}$ is given by (7.22).

For $P$ to be an effective preconditioner, $P^{-1} r$ has to be easily computable for any given vector $r$. It is clear that $P^{-1}$ $=W^{\top} \Lambda_{P}^{-1} W$ is a piecewise constant function in the wavenumber domain. The preconditioning procedure

$$
P^{-1} r=W^{\top} \Lambda_{p}^{-1} W r
$$

consists therefore of three steps: decomposition, scaling, and synthesis, which are represented here by multiplications by $W, \Lambda_{p}^{-1}$ and $W^{T}$, respectively. To clarify this comment, we can rewrite (7.24) as

$$
P^{-1} r=\left(\sum_{l=1}^{L} \frac{1}{c_{l}} W_{l}^{T} W_{l}\right)
$$

where $W_{l}, 1 \leq l \leq L$, are $(N-1)^{2}$ square matrices which have the same $2^{l-1}$ to $2^{l}-1$ rows as $W$ and zero vectors for remaining rows. Then, the multiplications by $W_{1}$ and $W_{l}^{T}$ in the decomposition and synthesis steps of (7.25) can be implemented with FFTs and inverse FFTs. This is due to the fact that $W_{1}$ is a mapping from the space domain to the wavenumber domain, whereas $W T$ is a mapping from the wavenumber domain to the space domain. Using this technique, 
we obtain a preconditioner implementation similar to the fast Poisson solver of Section IV.

Let $F_{l}^{i d}=W_{i}^{T} W_{l}$. Then, $F_{l}^{i d}$ is a mapping from the space domain to the space domain. In addition, we have

$$
F_{i}^{i d}=W^{\top} \Lambda_{F_{l}^{i d}} W
$$

where $\Lambda_{F_{l}^{i d}}$ is a diagonal matrix whose $k$ th element is

$$
\hat{F}_{l}^{i d}(k)= \begin{cases}1, & k \in B_{l} \\ 0, & \text { otherwise. }\end{cases}
$$

We see that the $F_{i}^{i d}$ functions as an ideal bandpass filter for the band $B_{1}$. Although it is possible to implement the ideal bandpass characteristic (7.26b) with FFTs or bandpass filters of size $N$, the resulting implementations either cannot be extended to more general PDEs, or are too expensive (i.e., or complexity $O\left(N^{2}\right)$ ). This leads us to approximate the ideal bandpass filter $F_{l}^{i d}$ with a nonideal filter $F_{l}$ with

$$
\hat{F}_{l}(k) \approx \begin{cases}1, & k \in B_{l} \\ 0, & \text { otherwise }\end{cases}
$$

so that $F_{l}$ can be implemented cost effectively for general problems.

An implementation of the preconditioner (7.25) would then consist of using digital filters to realize $F_{1}$ in the decomposition step, followed by a simple addition for the synthesis step. However, the decomposition and synthesis steps would be asymmetric, which is an undesirable feature in the multigrid context. This motivates us to write (7.25) differently as

$$
P^{-1} r=\left(\sum_{l=1}^{L} \frac{1}{\mathrm{C}_{l}} F_{l}^{T} F_{l}\right) r .
$$

The block diagram of Fig. 16 describes a procedure for constructing the bandpass filters $F_{l}$, with $1 \leq I \leq L$, in terms of a cascade of elementary low-pass filters $H_{L}, H_{L}-1$, $\cdots, H_{2}$. From Fig. 16, we see that $F_{1}$ can be expressed in terms of the filters $H_{1}$ as

$$
F_{L}=I-H_{L}
$$

$$
\begin{aligned}
& F_{1}=\left(I-H_{1}\right)\left[\prod_{\rho=l+1}^{L} H_{p}\right], \quad 2 \leq I \leq L-1 \\
& F_{1}=\prod_{p=2}^{L} H_{\rho} .
\end{aligned}
$$

Let the elementary filter $H_{L}$ be an FIR filter of the form

$$
H_{L}=a_{0}+\sum_{j=1}^{\prime} a_{j}\left(E^{j}+E^{-j}\right)
$$

where the coefficients $a_{j}$ are selected so that the spectrum $\tilde{H}_{L}(k)$ approximates an ideal lowpass filter, i.e.,

$$
\hat{H}_{L}(k) \approx \begin{cases}1, & 0 \leq k<2^{L}-1 \\ 0, & 2^{L-1} \leq k \leq 2^{L} .\end{cases}
$$

The coefficients $a_{j}$ can be determined by using any standard digital low-pass filter design technique. One specific choice is examined in [71]. The same coefficients are also used for constructing the /th-level elementary filter

$$
H_{l}=a_{0}+\sum_{j=1} a_{j}\left(E^{2^{l-1} j}+E^{-2^{L-1} j}\right)
$$

with $2 \leq l \leq L$. Comparing (7.30) and (7.32), we see that the only difference between elementary filters $H_{L}$ and $H_{l}$ is that while $H_{L}$ constructs a weighted average of points separated by a distance of $h$, the /th-level filter $H_{1}$ performs the same average over points separated by a distance of $2^{l-1} h$. Since some of the points needed to perform the above averages may be located outside the domain $\Omega_{h}$, the system (7.16) is viewed as defined on an infinite grid with an odd-periodic extended driving function, i.e.,

$$
f_{-n}=-f_{n} \quad \text { and } \quad f_{n+2 p N}=f_{n}
$$

for $p$ integer. The preconditioner shown in Fig. 16 is called the SGMF preconditioner.

The SGMF preconditioner of Fig. 16 can be simplified further by deleting paths corresponding to $I-H_{l}$. The resulting modified SGMF preconditioner is shown in Fig. 17. It can be expressed as

$$
Q^{-1} r=\left(\sum_{l=1}^{l} \frac{1}{d_{l}} G_{l}^{I} G_{l}\right) r
$$

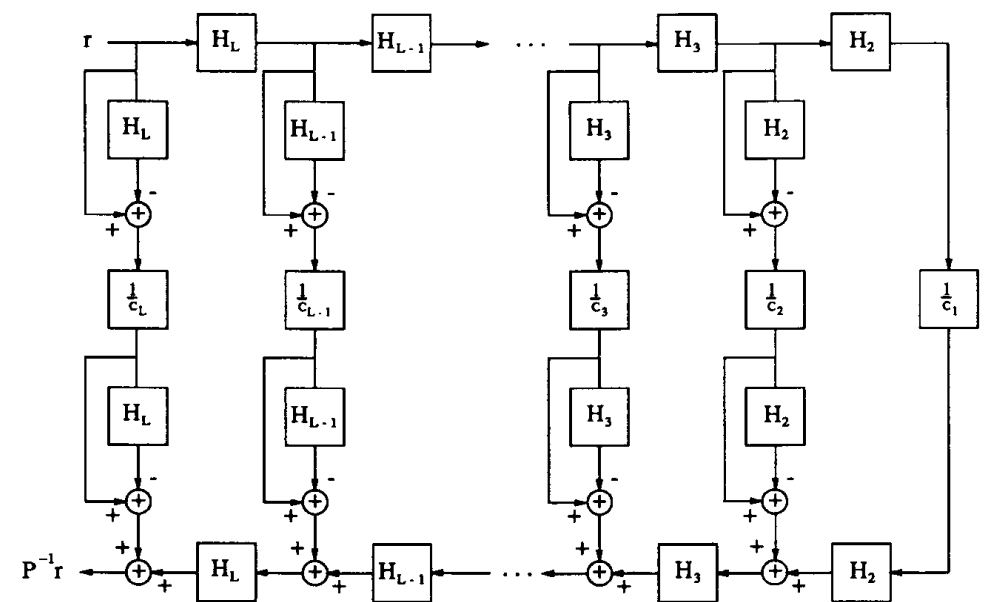

Fig. 16. Block diagram of the MF preconditioned with a single discretization grid (SGMF). 


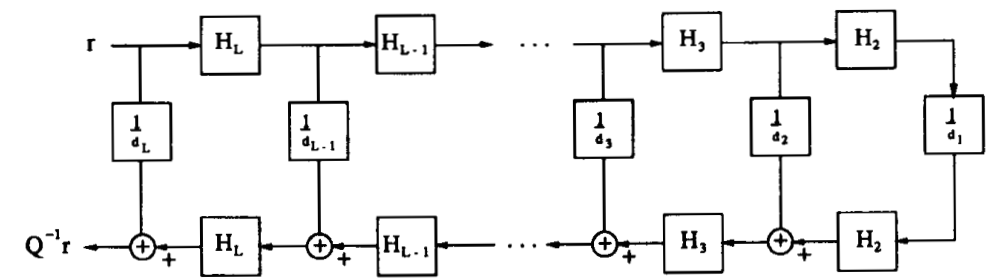

Fig. 17. Block diagram of the modified SGMF preconditioner.

with

$$
\begin{aligned}
& G_{L}=I, \\
& G_{l}=\prod_{p=I=1}^{L} l_{p}^{p-1} H_{p}, \quad 1 \leq I \leq L-1,
\end{aligned}
$$

and where the scaling constants $d_{l}$ are related to the constants $c_{l}$ via

$$
\sum_{l=1}^{l} \frac{1}{d_{i}}=\frac{1}{c_{l}}
$$

Note that unlike the preconditioner $P$, which relied on bandpass filters $F_{l}$, the modified preconditioner $Q$ is implemented in terms of lowpass filters $G_{1}$. A consequence of this feature is that the wavenumber components of the residual $r$ belonging to the band $B_{l}$ are present at the first $L-I+$ 1 levels. Since according to Fig. 18, these components are multiplied by $d_{l}^{-1}, \cdots, d_{l}^{-1}$ respectively, the preconditioners $Q$ and $P$ will be equivalent only if the constants $c_{i}$ and $d_{\text {, }}$ satisfy the relation (7.36).

The filtering operations that we have just described are performed at every grid point, for all levels $2 \leq I \leq L$. If the order $J$ of filters $H_{1}$ is finite, the number of operations required for such an implementation is proportional to $O(N$ $\log N)$, where $N$ is the total number of unknowns. However, since waveforms consisting only of low wavenumber components can be represented accurately on coarser grids, we can incorporate the multigrid structure into the above framework. This is illustrated in Fig. 18, which we call the MGMF preconditioner. Note that the MGMF preconditioner is obtained by inserting 2:1 down-samples $\left(I_{l}^{l-1}\right)$ and $1: 2$ up-samples $\left(I_{t-1}^{\prime}\right)$ into the modified SGMF preconditioner. It is easy to see that the number of operations required by the MGMF preconditioner if proportional to $O(N)$ instead of $O(N \log N)$ for the SGMF case.

The generalization of the MF preconditioner to multidimensional problems on regular domains is straightforward. For example, the 2-D elementary filter $H_{1}$ can be obtained as the tensor product of 1-D elementary filters along the $x$-and $y$-directions. It has been shown by Fourier analysis that the condition number of the MF-preconditioned Laplacian implemented with nonideal filters is proportional to $O(1)$ for the 1-D, 2-D and 3-D cases. This implies that the MF-CG method converges in a finite number of iterations independently of $h$, which has been confirmed by numerical experiments [71], [95].

\section{Historical Notes}

The conjugate gradient method for solving linear systems of equations was developed in late 1940 s and early $1950 \mathrm{~s}$ by Hestenes, Stiefel, and others. For a history of the conjugate gradient algorithm and the closely related Lanczos algorithm, the readers are referred to a recent survey by Golub and O'Leary [49], which contains an annotated bibliography for the period 1948-1976. A detailed presentation of the SSOR, ILU, and MILU preconditioners can be found in the book by Axelsson and Barker [7]. The Fourier analysis of the ILU, MILU and SSOR preconditioners for the naturally ordered Poisson problem with periodic boundary conditions was performed by Chan and Elman [24]. They also observed strong similarities in the eigenvalue distribution of incomplete factorization preconditioners for the Dirichlet and periodic problems. Kuo and Chan [70] used two-color Fourier analysis to study the eigenvalue distribution of the ILU, MILU, and SSOR preconditioned Laplacian with the red-black ordering. In the last few years, a growing amount of work has focused on the design of multilevel preconditioners. A brief survey of recent advances in this area can be found in the papers by Kuo, Chan, and Tong [71] and by Axelsson and Vassilevski [11].

\section{Domain Decomposition Method}

Domain decomposition methods rely on a partition of the domain of definition $\Omega$ of a given PDE into subdomains $\mathbf{\Omega}_{i}$ with or without overlapping regions. The original prob-

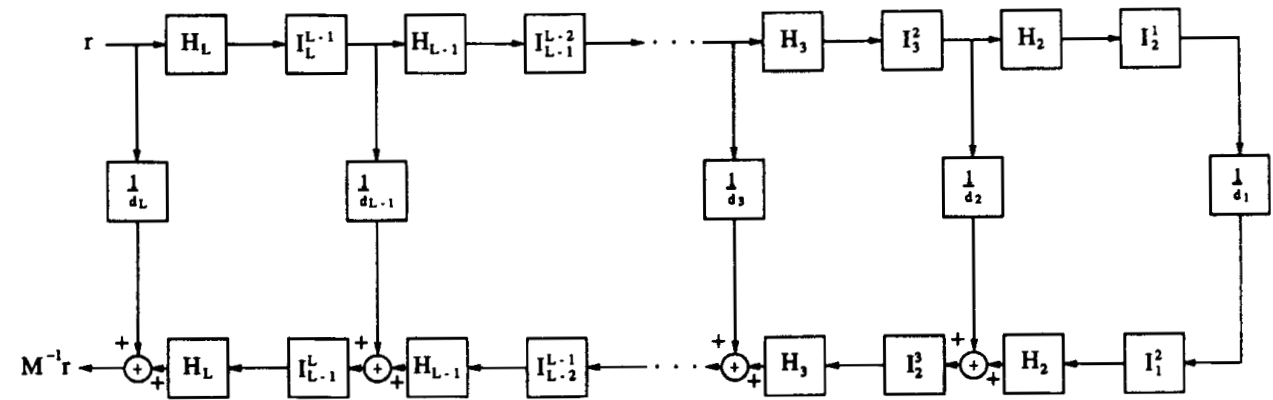

Fig. 18. Block diagram of the MGMF preconditioner. 
lem is then decomposed into smaller problems defined over each subdomain, which can be solved independently, provided that a strategy is developed for evaluating the variables corresponding to overlapping regions, or to interfaces between subdomains. Domain decomposition technique present several advantages. First, it is often possible to select the subdomain $\Omega_{i}$ in such a way that special solvers, such as fast direct solvers or MG methods, can be applied to the subproblems, even though they are not applicable to the problem defined over the entire domain $\Omega$. This is the case for example when $\Omega$ is irregular, but can be represented as the union of regular subdomains $\Omega_{i}$, or when the PDE has constant parameters over each subdomain, but not over the entire domain, such as for interface problems between different materials. Domain decomposition methods are also attractive from the point of view of parallel computation, since all subproblems can be solved in parallel.

Domain decomposition algorithms can be divided into two categories, depending on whether the subdomains overlap or not. Algorithms with overlapping subdomains fall into the category of Schwartz alternating methods [89], whereas those with nonoverlapping subdomains are called iterative substructuring or capacitance matrix methods. We restrict our attention here to capacitance matrix methods, where the domain is decomposed into regular subdomains, and the capacitance system governing the variables on the interfaces between subdomains is solved by an iterative method, such as the PCG algorithm. Since each iteration requires the solution of problems over each subdomain, it is important to find good preconditioners for the capacitance system. To do so, we use Fourier analysis to study the capacitance system corresponding to a simple model problem consisting of Poisson's equation defined over a rectangle divided horizontally into two subrectangles. This analysis leads to FFT-based preconditioners, which are then shown to be effective for more complex domain geometries.

\section{A. Capacitance Matrix Formulation}

Consider a discretized elliptic PDE with Dirichlet boundary conditions,

$$
A u=f
$$

whose domain $\Omega$ is partitioned into two nonoverlapping subdomains $\Omega_{1}$ and $\Omega_{2}$ with an interface region $\Gamma_{3}$, as shown in Fig. 19. By partitioning the solution $u$ and driving function $f$ into subvectors $u_{i}$ and $f_{i}$, with $i=1,2,3$, corresponding to the unknowns and driving terms indexed by points of $\Omega_{1}, \Omega_{2}$, and $\Gamma_{3}$, respectively, (8.1) can be expressed in block

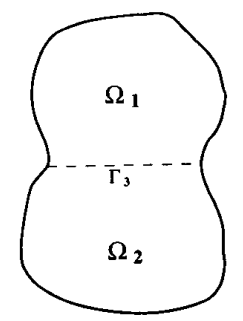

Fig. 19. A general domain and its partitioning. form as

$$
\left[\begin{array}{lll}
A_{11} & & A_{13} \\
& A_{22} & A_{23} \\
A_{13}^{T} & A_{23}^{T} & A_{33}^{T}
\end{array}\right]\left(\begin{array}{l}
u_{1} \\
u_{2} \\
u_{3}
\end{array}\right)=\left(\begin{array}{l}
f_{1} \\
f_{2} \\
f_{3}
\end{array}\right) .
$$

Using block Gaussian elimination, the system (8.2) can be solved as follows:

Step 1: Determine $u_{3}$ by solving the capacitance system

$$
\mathrm{Cu}_{3}=\mathrm{g}_{3}
$$

where the capacitance matrix

$$
C=A_{33}-A_{13}^{T} A_{11}^{-1} A_{13}-A_{23}^{r} A_{22}^{-1} A_{23}
$$

is the Schur complement of $\operatorname{diag}\left(\mathrm{A}_{11}, A_{22}\right)$ inside $A$, and

$$
g_{3}=f_{3}-A_{13}^{T} A_{11}^{-1} f_{1}-A_{23}^{T} A_{22}^{-1} f_{2} \text {. }
$$

Step 2: Compute $u_{1}$ and $u_{2}$ from

$$
u_{1}=A_{11}^{-1} g_{1} \quad u_{2}=A_{22}^{-1} g_{2}
$$

with

$$
g_{1}=f_{1}-A_{13} u_{3} \quad \text { and } \quad g_{2}=f_{2}-A_{23} u_{3} . \quad \text { (8.6b) }
$$

In (8.5) and (8.6), we need to invert the matrices $A_{11}$ and $A_{22}$ which describe the coupling among variables of subdomains $\Omega_{2}$ and $\Omega_{2}$, respectively. The operation $A_{11}^{-1} w$ (or $\left.A_{22}^{-1} w\right)$, where $w$ is an appropriate vector, is called a subproblem solve. It can often be implemented by using fast direct or MG methods. The solution of the capacitance system (8.3) is more difficult. It is usually not desirable to form the capacitance matrix $C$ explicitly, since the direct computation of the elements of $C$ is very expensive. Instead, when (8.3) is solved by iterative methods such as the PCG algorithm, only the computation of $\mathrm{CW}$ is required, which involves two subproblem solves. Due to the high cost of computing $C w$, it is important that iterative methods should converge very fast. Consequently, the design of good preconditioners for the capacitance matrix $C$ is the key to the development of efficient nonoverlapping domain decomposition algorithms.

\section{B. Fourier Analysis of the Capacitance System}

As a first step, we consider the case where the matrix $A$ in (8.1) represents the 5-point discretized Laplacian with local operator (3.7), defined over a rectangular domain $\Omega$. We also assume that $\Omega$ is decomposed horizontally into two rectangular strips $\Omega_{1}$ and $\Omega_{2}$, as shown in Fig. 20. In the

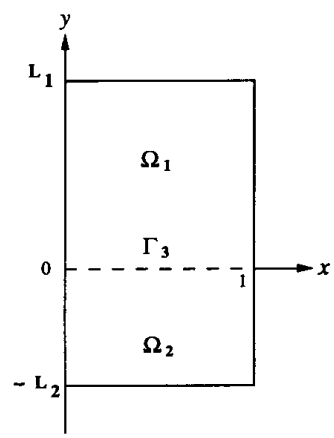

Fig. 20. A rectangular domain and its partitioning. 
$\boldsymbol{x}$-direction, $\mathbf{\Omega}$ is discretized uniformly with mesh size $h=$ $N^{-1}$, where $N-1$ is the number of internal discretization points. In the $y$-direction, we assume that the widths $L_{1}$ and $L_{2}$ of $\Omega_{1}$ and $\Omega_{2}$ satisfy

$$
L_{1}=M_{1} h \quad \text { and } \quad L_{2}=M_{2} h
$$

where $M_{1}$ and $M_{2}$ are positive integers.

A consequence of this simple decomposition geometry is that Fourier analysis can be employed to study the capacitance system (8.3). Specifically, we show below that the matrices $A_{33}, A_{13}^{T} A_{11}^{-1} A_{13}$ and $A_{23}^{T} A_{22}^{-1} A_{23}$ appearing in the definition (8.4) of $C$ all have for eigenvectors the sine vectors

$$
\begin{gathered}
w_{k}^{T}=\sqrt{2} h(\sin (k \pi h), \cdots, \sin (k \pi n h), \cdots, \\
\sin (k \pi(N-1) h))
\end{gathered}
$$

with $1 \leq k \leq N-1$.

First, the local operator corresponding to $A_{33}$ can be expressed as

$$
1-\frac{1}{4}\left(E_{x}+E_{x}^{-1}\right) .
$$

Consequently, by operating with $A_{33}$ on $w_{k}$, we obtain

$$
A_{33} w_{k}=\left[1-\frac{1}{2} \cos (k \pi h)\right] w_{k}=\frac{1}{4}\left(2+\sigma_{k}\right) w_{k}
$$

with

$$
\sigma_{k}=4 \sin ^{2}\left(\frac{k \pi h}{2}\right)
$$

Thus, $w_{k}$ is an eigenvector of $A_{33}$

Next, we examine $-A_{13}^{T} A_{11}^{-1} A_{13}$. Let $A_{11}^{-1} A_{13} w_{k} \equiv v_{k}$, so that

$$
A_{11} v_{k}=A_{13} w_{k}
$$

The equation (8.11) can be viewed as obtained by discretizing Laplace's equation (the driving function is zero) on $\mathrm{n}_{1}$ with zero boundary values along the east, north and west boundaries and $w_{k}$ along the south boundary. It turns out that its solution $v_{k}$ admits the closed-form expression

$$
v_{k}\left(n_{x}, n_{y}\right)=\sqrt{2 h} \sin \left(k n_{x} \pi h\right) d_{k, 1}\left(n_{y}\right)
$$

where $d_{k, 1}\left(n_{y}\right)$ satisfies the difference equation

$$
\begin{gathered}
d_{k, 1}\left(n_{y}-1\right)-\left(2+\sigma_{k}\right) d_{k, 1}\left(n_{y}\right)+d_{k, 1}\left(n_{y}+1\right)=0, \\
1 \leq n_{y} \leq M_{1}-1
\end{gathered}
$$

with boundary conditions $d_{k, 1}(0)=1$ and $d_{k, 1}\left(M_{1}\right)=0$. We are interested here in the quantity

$$
-A_{13}^{\top} A_{11}^{-1} A_{13} w_{k}=-A_{13}^{T} v_{k}=\frac{1}{4} v_{k}\left(n_{x}, n_{y}=1\right)=\frac{1}{4} d_{k, 1}(1) w_{k} .
$$

Thus, $w_{k}$ is an eigenfunction of $-A_{13}^{I} A_{11}^{-1} A_{13}$ with eigenvalue $d_{i, 1}(1) / 4$. The same procedure can be used to analyze the matrix $-A_{23}^{T} A_{22}^{-1} A_{23}$. This gives

$$
-A_{23}^{T} A_{22}^{-1} A_{23} w_{k}=\frac{1}{4} d_{k, 2}(1) w_{k}
$$

where $d_{k, 2}(1)$ is obtained by solving

$$
\begin{gathered}
d_{k, 2}\left(n_{y}-1\right)-\left(2+\sigma_{k}\right) d_{k, 2}\left(n_{y}\right)+d_{k, 2}\left(n_{y}+1\right)=0, \\
1 \leq n_{y} \leq M_{2}-1
\end{gathered}
$$

with boundary conditions $d_{k, 2}(0)=1$ and $d_{k, 2}\left(M_{2}\right)=0$. Combining (8.9), (8.14), and (8.15) yields

$$
C w_{k}=\frac{1}{4}\left(2+\sigma_{k}+d_{k, 1}(1)+d_{k, 2}(1)\right) w_{k} \equiv \lambda_{k} w_{k}
$$

for $1 \leq k \leq N-1$, so that $w_{k}$ is an eigenvector of $C$, as claimed. Further analysis shows [23] that the eigenvalue $\lambda_{k}$ associated to $w_{k}$ can be expressed as

$$
\lambda_{k}=g\left(k, M_{1}, M_{2}\right) \sqrt{\sigma_{k}+\frac{1}{4} \sigma_{k}^{2}}
$$

where $\sigma_{k}$ is given by (8.10), and

$$
g\left(k, M_{1}, M_{2}\right)=\frac{1}{4}\left(\frac{1+\gamma_{k}^{M_{1}}}{1-\gamma_{k}^{M_{1}}}+\frac{1+\gamma_{k}^{M_{2}}}{1-\gamma_{k}^{M_{2}}}\right)
$$

with

$$
\gamma_{k}=\left(1+\frac{1}{2} \sigma_{k}-\sqrt{\sigma_{k}+\frac{1}{4} \sigma_{k}^{2}}\right)^{2} .
$$

Note that $\sigma_{k}$ is the spectrum of the 1-D Laplacian operator $L=2-\left(E_{x}+E_{x}^{-1}\right)$ defined on $\Gamma_{3}$. The respective spectra $\lambda_{k}$ and $\sigma_{k}$ of the capacitance matrix $C$ and Laplacian $L$, and the function $g\left(k, M_{1}, M_{2}\right)$, are plotted in Fig. 21 for $M_{1}=M_{2}=$ 40 and $h^{-1}=256$.

The geometric parameters $M_{1}$ and $M_{2}$ which specify the sizes of subdomains $\Omega_{1}$ and $\Omega_{2}$ affect only the function $g(k$, $M_{1}, M_{2}$ ). From Fig. 21, we see that this function has values

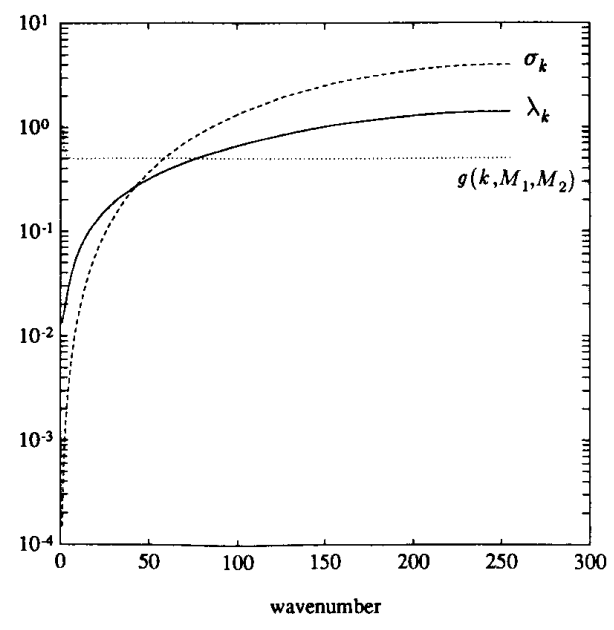

Fig. 21. Plots of $\lambda_{k}, \sigma_{k}$ and $g\left(k, M_{1}, M_{2}\right)$ as functions of the wavenumber $k$.

of $O(1)$. For large $M_{1}$ and $M_{2}$ with fixed $k, g\left(k, M_{1}, M_{2}\right)$ reaches its asymptotic value 0.5 rapidly. Therefore, $(8.18)$ can be simplified as

$$
\lambda_{k} \approx 0.5 \sqrt{\sigma_{k}+\frac{1}{4} \sigma_{k}^{2}}
$$

Since $\sigma_{k}^{2} \ll \sigma_{k}$ for small $k$ and $\frac{1}{4} \sigma_{k}^{2} \approx \sigma_{k}$ for large $k$, an even rougher estimate for $\lambda_{k}$ is

$$
\lambda_{k} \approx 0.5 \sqrt{\sigma_{k}} \text {. }
$$

In summary, we have shown in this section that if $W$ is the orthonormal matrix of size $N-1$ whose columns are the sine vectors $w_{k}$, the capacitance matrix $C$ associated to the partition of a rectangular domain into two horizontal 
strips admits the eigenvalue/eigenvector decomposition

$$
\begin{aligned}
& C=W \Lambda W^{T} \quad \text { with } \\
& \Lambda=\operatorname{diag}\left\{\lambda_{1}, \cdots, \lambda_{k}, \cdots, \lambda_{N-1}\right\} .
\end{aligned}
$$

\section{Preconditioners for the Capacitance Matrix}

From (8.22) and (8.18), it is easy to check that, for $h$ sufficiently small, the condition number of the capacitance matrix $C$ is given by

$$
\kappa(C)=\frac{\max \lambda_{k}}{\min \lambda_{k}} \approx \frac{2 \sqrt{2}}{S \pi h}=O\left(h^{-1}\right)
$$

with

$$
S=\frac{1}{2}\left(\frac{1+e^{-2 \pi L_{1}}}{1-e^{-2 \pi L_{1}}}+\frac{1+e^{-2 \pi L_{2}}}{1-e^{-2 \pi L_{2}}}\right)
$$

It is therefore of interest to design preconditioners $M$ such that $\kappa\left(M^{-1} C\right)=O(1)$. Several such preconditioners have been proposed in the literature. These preconditioners are all of the form

$$
M=W D W^{T}
$$

and differ only by the choice of diagonal matrix $D$. Dryja [33], and Golub and Mayers [48] proposed preconditioners with

$$
\begin{aligned}
& D_{D}=0.5 \operatorname{diag}\left\{\sqrt{\sigma_{k}}\right\} \quad \text { and } \\
& D_{G}=0.5 \operatorname{diag}\left\{\sqrt{\sigma_{k}+\frac{1}{4} \sigma_{k}^{2}}\right\}
\end{aligned}
$$

respectively. These preconditioners can be motivated by the eigenvalue decomposition (8.22) for $C$, and approximations (8.21) and (8.20), respectively, for the eigenvalues $\lambda_{k}$ of $C$. More recently, Chan [23] proposed the selection of

$$
D_{\mathrm{C}}=\Lambda
$$

where $\lambda$ is given by (8.22). The preconditioner $M$ given by (8.24), (8.25b) is exact for Poisson's equation and the domain decomposition geometry of Fig. 20. Finally, observe that all preconditioners of the form (8.24) admit FFT implementations.

An interesting feature of the above preconditioners is that, although they were designed for the case where $\Omega$ is a rectangle divided horizontally into two subrectangles, they are applicable to complex domain geometries where $\Omega$ is the union of an arbitrary number of rectangles. Consider for example the Poisson equation defined on the $L$ or C-shaped regions of Figs. 22(a) and 22(b). For the $L$-shaped domain of Fig. 22(a), $\Omega$ can be viewed as obtained by assembling the three elementary rectangles $\Omega_{i}$ with $i=1,2,3$. The corresponding interfaces are $\Gamma_{4}$ and $\Gamma_{5}$. Consider now a decomposition of $\Omega$ into two rectangles $\Omega_{1}$ and $\Omega_{23}=\Omega_{2} U$ $\Omega_{3}$. The corresponding capacitance system defined over interface $\Gamma_{4}$ is

$$
\mathrm{C}_{4} u_{4}=g_{4} .
$$

To precondition this system, we can ignore the presence of $\Omega_{3}$, and let $M_{4}$ be the preconditioner given by (8.24), (8.25b) when we partition $\Omega_{12}=\Omega_{1} \cup \Omega_{2}$ into $\Omega_{1}$ and $\Omega_{2}$ with interface $\Gamma_{4}$. It was shown by Chan and Resasco [27] that with this choice, the condition number $\kappa\left(M_{4}^{-1} C_{4}\right)$ is $O(1)$. A similar result holds for the $C$-shaped domain geometry of Fig. 22(b)

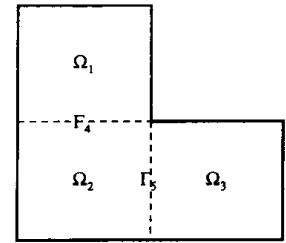

(a)

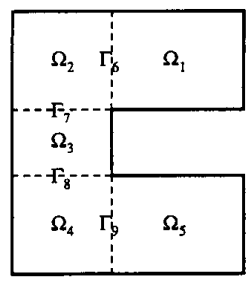

(b)

Fig. 22. (a) L-shaped and (b) C-shaped domains and their partitionings.

[27]. This indicates that preconditioners designed for rectangular domains remain effective for more complex domain geometries. More generally, for an arbitrary problem such as the one depicted in Fig. 19, one may fit the domain with two subrectangles in such a way the geometric parameters $M_{1}$ and $M_{2}$ can be estimated, and then used to design a preconditioner of the form (8.24)-(8.25).

\section{Historical Notes}

The first domain decomposition technique for solving elliptic problems was introduced by Schwartz in 1869, who proposed an alternating procedure, where the problem is solved by going in alternance from one subdomain to another. A short history of the early work on domain decomposition methods can be found in [98]. The recent interest in domain decomposition techniques is due to the fact that these methods are intrinsically parallel, and are therefore well adapted to parallel computers. A recent paper by Keyes and Gropp [68] provides a good introduction to domain decomposition methods for readers unfamiliar with this topic. It gives an overview of various domain decomposition techniques, compares their performance, and discusses their parallel implementation. The Fourier analysis of the capacitance matrix for a rectangular domain divided into two subrectangles was first proposed by Chan [23]. The extension of this analysis to the case of a rectangle divided into an arbitrary number of rectangular strips is described in [26]. In [27], [28], Chan and Resasco presented a general framework for the analysis and construction of domain decomposition preconditioners over irregular regions. For a more general perspective on domain decomposition methods, and on their application to a wide variety of PDEs, readers may wish to consult the preceedings of two conferences on domain decomposition methods held in 1987 and 1988 [47], [25].

\section{Parallel Computation}

There has been much progress during the last 20 years in developing vector and parallel computer architectures [61], [62] and algorithms for solving elliptic PDEs. In this sec- 
tion, we focus on algorithms for parallel computers and will give a brief account of the main achievements in this area. For a more thorough review, we refer readers to the work of Ortega and Voigt [86], [87].

As indicated in Section III, one way to parallelize PDE algorithms is to reorder the sequence of grid points to be processed in such a way that a large number of operations can be performed in parallel. For example, the red-black ordering is more attractive than the natural ordering for solving 5-point discretized elliptic PDEs, as far as parallel implementation is concerned. One interesting question that arises in this context is whether the convergence rate of iterative algorithms is affected by the reordering scheme. This problem has been studied in [1], [38], [70], [75], [77]. In particular, the effect of the red-black ordering on SOR and PCG algorithms is discussed in detail in [70]. Briefly speaking, the convergence rate of the SOR algorithm is independent of ordering schemes, but the convergence rate of PCG algorithms depends on the choice of ordering. For the CG method preconditioned by the MILU or SSOR method, the convergence rate of the red-black ordering is one order of magnitude slower than that of the natural ordering [38], [70]. For PCG methods, there exists therefore a tradeoff between the rate of convergence and the degree of parallelism that can be achieved.

No such tradeoff exists for the SOR method, but another difficulty arises when one seeks to implement it in parallel. Specifically, when the coefficients of the PDE are spacedependent, the optimal relaxation parameter depends in general on global information and must be estimated adaptively [57]. The estimation of the relaxation parameter requires global communication between all processors, a feature that slows down the SOR algorithm significantly. To overcome this difficulty, a local relaxation procedure was proposed in [16], [37], [75] where different relaxation parameters are used at every grid point, and are determined on the basis of local information. Since, unlike the conventional SOR algorithm, no global information is needed for determining the optimal local relaxation parameters, the communication time between multiple processors is significantly reduced. Another extension of the red-black SOR algorithm involves the use of more than two colors for ordering the grid points. The motivation for considering multiple coloring schemes is that when elliptic PDEs are discretized on high-order stencils, more than two colors are necessary to decouple all grid points of the same color. For the case of a 9-point stencil discretization, four colors are needed. The extension of the red-black SOR algorithm to multiple coloring schemes can take different forms. For the 9-point discretized Poisson problem, two such extensions have been proposed by Adams, Leveque, and Young [2], and by Kuo and Levy [73], which rely respectively on a single- or two-level relaxation scheme. Both of these methods are easily parallelizable on mesh-connected processor arrays.

In parallel implementations of the PCG algorithm, the major bottleneck is usually the parallelization of the preconditioner (7.4), since the remaining steps of the PCG algorithm can be parallelized in a straightforward way. The main difficulty lies in the fact that elliptic PDE problems involve a global coupling of all grid points. In order to be effective, preconditioners must take into account this global cou- pling by including a mechanism for transmitting information from one point of the problem domain to another. Consequently, preconditioners that use purely local information, such as the red-black ordered MILU and SSOR and polynomial preconditioners, are fundamentally limited in their ability to improve the convergence rate of the $\mathrm{CG}$ algorithm. On the other hand, global coupling through a natural ordering grid traversal is not highly parallelizable. To construct highly parallelizable and effective preconditioners, we are therefore led to consider preconditioners which share global information through a multilevel grid structure, thus ensuring a good convergence rate, but perform only local operations on each grid level, and hence are highly parallelizable. Preconditioners that have this feature include the multigrid method when used as a preconditioner [66], [67], and the hierarchical basis basis preconditioner [104], [105]. More recently, new multilevel preconditioners have been proposed by Bramble, Pasciak, and Xu [17], [99] and Kuo, Chan, and Tong [71]. These preconditioners differ from multgrid methods by the fact that the smoothing operation in multigrid methods is replaced by a simple scaling operation, as was shown in Section VII-B. Other types multilevel preconditioners have been examined in [6], [9], [10], [76], [97]. A detailed comparison of several multilevel elliptic preconditioners can be found in [71].

The parallelization of multigrid methods or multilevel preconditioners on multiprocessor machines is one of the most challenging areas in parallel computing for elliptic PDEs. A significant amount of work has focused on parallelizing standard multigrid algorithms on mesh-connected arrays [19], [44] and hypercubes [29]. Variants of standard multigrid algorithms aiming at achieving more parallelism on massively parallel computers have also been proposed. These parallel multigrid algorithms include the concurrent multigrid method [44] and the superconvergent multigrid method [43]. A survey of developments in this field up to 1987 is presented in [30]. More recent contributions can be found in [79]. Roughly speaking, two fundamental issues arise in parallelizing multigrid methods. One is to find an appropriate mapping which assigns adjacent grid points to neighboring processors so that only local communication is required. Since the hierarchy of grids in the multigrid algorithm complicates the flow of data, this is in general not easy. However, for the hypercube machine this mapping problem has been solved by Chan and Saad [29]. The second problem is usually known as that of load balancing. To get maximal parallelism, we need as many processors as there are points at the fine grid level. However, when relaxation is performed on the coarse grid, the majority of the processors become idle. Thus, the problem is to reduce the number of idle processors as much as possible so that the efficiency of the entire multiprocessor system is maximized. One promising way to solve this problem is to perform concurrent iterations at different grid levels. For example, we may use filtering to split the problem into multiple subproblems defined on different grids, where each subproblem corresponds to a different spectral component of the original problem. These subproblems could then be solved simultaneously by performing concurrent relaxations on all grids. However this approach raises many questions: what is the optimal splitting scheme? What is the best filter for dividing a given problem into subproblems? 
How is the convergence and efficiency of standard multigrid algorithms affected by this decomposition procedure?

Domain decomposition provides a natural way to achieve parallel computation. This approach is particularly suitable for a coarse grain parallel computing environment where there are considerably fewer processors than grid points. One important issue in domain decomposition is the selection of the number of subdomains. On one hand, more subdomains imply more parallelism. On the other hand, the communication cost per iteration and the overall number of iterations tend to increase with the number of subdomains. Thus, the answer is generally architecture- and problem-dependent. The complexity of parallel implementations of domain decomposition techniques on a ring, a twodimensional mesh, and an n-cube has been studied by Gropp and Keyes [52]. Some performance analysis results and numerical experiments have also been reported in [21], [53], [58].

\section{Conclusion and Extensions}

Digital signal processing (DSP) and the numerical solution of PDEs have been traditionally considered as separate research areas. However, during the last 30 years Fourier analysis has been used increasingly by numerical analysts to analyze and design numerical PDE algorithms. Without surprise, results obtained by Fourier analysis can be reformulated within the DSP framework. Recently research work [69], [71], [72], [73], [75] has focused on bridging the gap between these two separate research areas, and a number of interesting new results have been obtained as a consequence of this effort. In this paper, we have described in detail the link existing between DSP and the numerical solution of PDEs, so that numerical PDE algorithms can be understood by electrical engineers in a more familiar setting. In addition, a number of recent developments on iterative solution techniques for elliptic PDEs have been reviewed so as to provide readers with the most up-to-date knowledge in this area.

The effort to bridge the gap between DSP and numerical differential equations will benefit researchers in both areas. From the electrical engineering side, researchers will be able to study existing numerical algorithms for different equations more easily. They will also find numerous interesting and challenging problems in the solution of differential equations, for example, the solution of PDEs consisting of both space and time variables. From the numerical analysis side, researchers will have new set of tools to analyze and design numerical algorithms. Further advances based on this connection can be expected in the future.

It is worthwhile to emphasize that the DSP approach relies on tools that are usually not used in the matrix context: the theory of multidimensional signals and systems [34] and frequency-domain analysis. To form a matrix equation, a 1-D ordering is required and, therefore, the proximity of grid points in multidimensional meshes is disguised. This phenomenon does not occur for multidimensional DSP techniques, since they are fully adapted to the spatial nature of the signals being studied. The discretized system of equations for the elliptic problem is loosely coupled in the space domain, but totally decoupled in the frequency domain. In other words, transforming the system from the space domain to the frequency domain corresponds to a diagonalization procedure whereby a sparse matrix is transformed into a diagonal matrix, thus leading to a much simpler analysis. Due to its simplicity, the DSP approach provides some valuable insight into the choice of solution method, as well as some guidelines towards the development of more versatile and efficient solution techniques. This point has been demonstrated in the application of digital filtering theory to the design of elliptic preconditioner as discussed in Section VII. Thus, we conclude that the DSP approach can serve as complement to the classical matrix analysis, which is more generally applicable but less transparent.

In this tutorial paper, we have examined discretization schemes and solution methods for solving elliptic PDEs from the DSP viewpoint. We studied mode-dependent finite-difference schemes for three model elliptic PDE problems, i.e., the Poisson, Helmholtz, and convection-diffusion equations. The extension of mode-dependent discretization schemes to coupled differential equations and timedependent problems, such as hyperbolic and parabolic PDEs, is currently being investigated. We also reviewed various methods for solving self-adjoint positive definite elliptic PDEs modeled by the Poisson equation, including direct methods, elementary and accelerated relaxation methods, multigrid methods, preconditioned conjugate gradient methods, and the domain decomposition technique.

A limitation of the DSP/Fourier point of view that we have adopted here is that it is restricted primarily to finite-difference discretization methods. Although the rigorous applicability of Fourier analysis to finite-element methods remains in doubt, it was shown by Strang and Fix [40], [91] that the Fourier approach can provide useful insights into the accuracy and stability of finite-element schemes. We hope that these early results will ultimately lead to a complete frequency-domain theory of finite-element methods. Finally, we expect that the DSP viewpoint will also be helpful to develop new efficient algorithms for solving more difficult elliptic PDEs such as indefinite and nonself-adjoint problems modeled by the Helmholtz and convection-dif. fusion equations.

\section{REFERENCES}

[1] L. M. Adams and H. F. Jordan, "Is SOR color-blind?," SIAM J. Sci. Stat. Comput., vol. 7, pp. 490-506, 1986.

[2] L. M. Adams, R. J. LeVeque, and D. M. Young, "Analysis of the SOR iteration for the 9-point Laplacian," SIAM J. Numer. Anal., vol. 25, pp. 1156-1180, 1988.

[3] D. N. De G. Allen and R. V. Southwell, "Relaxation methods applied to determine the motion, in two dimensions, of a viscous fluid past a fixed cylinder," Q. J. Mech. and Appl. Math., vol. 8, pp. 129-145, 1955.

[4] S. F. Ashby, "Polynomial preconditioning for conjugate gradient methods," Ph.D. disertation, Dept. of Computer Science, Univ. of Iilinois, Urbana, IL, 1987.

[5] O.Axelsson, "A generalized SSOR method," BIT, vol. 13, pp. 443-467, 1972.

[6] -, "An agebraic framework for multilevel methods," Report 8820, Dept. of Mathematics, Catholic Univ., The Netherlands, 1988.

[7] O. Axelsson and V. A. Barker, Finite Element Solution of Boundary Value Problems. New York, NY: Academic Press, 1984. 
[8] O. Axelsson and G. Lindskog, "On the rate of convergence of the preconditioned conjugate gradient methods," Numer. Math., vol. 48, pp. 499-523, 1986.

[9] O. Axelsson and P. Vassilevski, "Algebraic multilevel preconditioning methods, l" Numer. Math., vol. 56, pp. 157$177,1989$.

[10] O. Axelsson and P. Vassilevski, "Algebraic multilevel preconditioning methods, II," Report 1988-15, Institute for Scient. Comput., Univ. of Wyoming, Laramie, Wyoming, 1988.

[11] - "A survey of multilevel preconditioned iterative methods," BIT, vol. 29, pp. 769-793, 1989.

[12] N. S. Bakhvalov, "On the convergence of a relaxation method with natural constraints on the elliptic operator," U.S.S.R. Comp. Math. and Math. Phys., vol. 6, pp. 101-135, 1966.

[13] G. Birkhoff and A. Schoenstadt, Eds., Elliptic Problem Sol. vers II. New York, NY: Academic, 1984.

[14] G. Birkhoff and R. E. Lynch, Numerical Solution of Elliptic Problems. Philadelphia, PA: SIAM, 1984.

[15] N. K. Bose, "Multidimensional systems: present state as an indicator of future prospects," in Multidimensional Systems: Theory and Applications, N. K. Bose, Ed. New York: IEEE Press, pp. 1-22, 1979.

[16] E. F. Botta and A. E. P. Veldman, "On local relaxation methods and their application to convection-diffusion equations," J. Comput. Phys., vol. 48, pp. 127-149, 1981.

[17] J. H. Bramble, J. E. Pasciak, and J. Xu, "Parallel multilevel preconditioners," Math. Comp., vol. 55, pp. 1-22, 1990.

[18] A. Brandt, "Multi-level adaptive solutions to boundary-value problems," Math. Comp., vol. 31, pp. 333-390,

[19] $\frac{1977 .}{-}$ "Multigrid solvers on parallel computers," in Elliptic Problem Solvers, M. H. Schultz, Ed. New York, NY: Academic, 1981, pp. 39-83.

[20] W. L. Briggs, A. Multigrid Tutorial, Philadelphia, PA: SIAM, 1987.

[21] L. Brochard, "Efficiency of multicolor domain decomposition on distributed memory systems," in Domain Decomposition Methods, T. F. Chan et al., Eds. Philadelphia, PA: SIAM, 1989, pp. 249-259.

[22] O. Buneman, "A compact non-iterative Poisson solver," Report 294, Stanford Univ., Institute for Plasma Research, Stanford, CA, 1969

[23] T.F. Chan, "Analysis of preconditioners for domain decomposition," SIAM J. Numer. Anal, vol. 24, pp. 382-390, 1987.

[24] T. F. Chan and H. C. Elman, "Fourier analysis of iterative methods for elliptic problems," SIAM Review, vol. 31, pp. 20-49, 1989.

[25] T. F. Chan, R. Glowinski, J. Periaux and O. B. Widlund, Eds. Domain Decomposition Methods, Philadelphia, PA: SIAM, 1989.

[26] T. F. Chan and D. C. Resasco, "A domain-decomposed fast Poisson solver on a rectangle," SIAM J. Sci. Stat. Comput., vol. 8, pp. 14-26, 1987

[27] - "Analysis of domain decomposition preconditioners on irregular regions," in Advances in computer methods for partial differential equations. VI, R. Vichnevetsky and R. S. Stepleman, Eds. New Brunswick, NJ: IMACS, Dept. of Computer Science, Rutgers Univ., 1987, pp. 317-322.

[28] — "A framwork for the analysis and construction of domain decomposition preconditioners," in First International Symposium on Domain Decomposition Methods for Partical Differential Equations, R. Glowinski et al., Eds. Philadelphia, PA: SIAM, 1988, pp. 217-230.

[29] T. F. Chan and Y. Saad, "Multigrid algorithms on hypercube multiprocessor," IEEE Trans. on Computers, vol. C-35, pp. 969-977, 1986.

[30] T. F. Chan and R. S. Tuminaro, "Design and implementation of parallel multigrid algorithms," in Multigrid Methods: Theory, Applications, and Supercomputing, S. F. McCormick, Ed. New York, NY: Marcel Dekker, 1988, pp. 101-115.

[31] R. E. Crochiere and L. R. Rabiner, Multirate Digital Signal Processing. Englewood Cliffs, NJ: Prentice-Hall, 1983.
[32] G. Dahlquist and A.Bjorck, Numerical Methods. Englewood Cliffs, NJ: Prentice-Hall, 1974

[33] M. Dryja, "A capacitance matrix method for Dirichlet problem on polygon region," Numer. Math., vol. 39, pp. 51-64, 1982.

[34] D. E. Dudgeon and R. M. Mersereau, Multidimensional Digital Signal Processing. Englewood Cliffs, NJ: Prentice-Hall, 1984.

[35] I. S. Duff, A. M. Erisman, and J. K. Reid, Direct Methods for Sparse Matrices. Oxford, UK: Oxford University Press, 1986.

[36] T. Dupont, R. P. Kendall, and J. H. H. Rachford, "An approximate factorization procedure for solving self-adjoint difference equations," SIAM J. Numer. Anal., vol. 5, pp. 559$573,1968$.

[37] L. W. Ehrlich, "An ad hoc SOR method," J. Comput. Phys., vol. 44, pp. 31-45, 1981

[38] $H$. Elman and E. Agron, "Ordering techniques for the preconditioned conjugated gradient method on parallel computers," Computer Physics Commun., vol. 53, pp. 253-269, 1989.

[39] R. P. Fedorenko, "The speed of convergence of an iterative process," U.S.S.R. Comp. Math. and Math. Phys., vol. 4, pp. 227-235, 1964.

[40] G. Fix and G. Strang, "Fourier analysis of the finite element method in Ritz-Galerkin theory," Studies in Appl. Math, vol. 48, pp. 265-273, 1969.

[41] D. Flanders and G. Shortley, "Numerical determination of fundamental modes," J. Appl. Phys., vol. 21, pp. 1326-1332, 1950.

[42] S. P. Frankel, "Convergence rates of iterative treatments of partial differential equations," Math. Tables Aids Comput., vol. 4 , pp. 65-75, 1950.

[43] P. O. Frederickson and O. A. McBryan, "Parallel superconvergent multigrid," in Multigrid Methods: Theory, Applications, and Supercomputing, S. F. McCormick, Ed. New York, NY: Marcel Dekker, 1988.

[44] D. Gannon and J. Van Rosendale, "On the structure of parallelism in a highly concurrent PDE solver," J. Parallel and Distributed Comput., vol. 3, pp. 106-135, 1986.

[45] W. Gautschi, "Numerical integration of ordinary differential equations based on trigonometric polynomials," Numer. Math., vol. 3, pp. 381-397, 1961.

[46] A. George and J. W. H. Liu, Computer Solution of Large Sparse Positive-Definite Systems. New Jersey: Prentice-Hall, 1981.

[47] R. Glowinski, G. Golub, G. A. Meurant, and J. Periaux, Eds., First Int. Symp. on Domain Decomposition Methods for Partial Differential Equations. Philadelphia, PA: SIAM, 1988.

[48] G. H. Golub and D. Mayers, "The use of preconditioning over irregular regions," lecture presented at the Sixth Int. Conf, on Computing Methods in Applied Sciences and Engineering, Versailles, 1983.

[49] G. H. Golub and D. P. O'Leary, "Some history of the conjugate gradient and Lanczos methods," SIAM Review, vol. 31, pp. 50-102, 1989.

[50] G. H. Golub and C. F. Van Loan, Matrix Computations, 2nd ed. Baltimore, MD: The John Hopkins Univ. Press, 1989.

[51] G. H. Golub and R. S. Varga, "Chebyshev semi-iterative methods, successive overrelaxation iterative methods, and second order Richardson iterative methods," Numer. Math., vol. 3, pp. 147-168, 1961.

[52] W. D. Gropp and D. E. Keyes, "Complexity of parallel implementations of domain decomposition techniques for elliptic partial differential equations," SIAM J. Sci. Stat. Comput., vol. 9, pp. 312-326, 1988.

[53] W. D. Gropp and D. E. Keyes, "Domain decomposition on parallel computers," in Domain Decomposition Methods, T. F. Chan et al., Eds. Philadelphia, PA.: SIAM, 1989, pp. 260-268.

[54] W. Hackbush, Multi-Grid Methods and Applications. Berlin, Germany: Springer-Verlag, 1985.

[55] W. Hackbusch and U. Trottenberg, Eds., Multigrid Methods. New York, NY: Springer-Verlag, 1982.

[56] - Multigrid Methods II. New York, NY: Springer-Verlag, 1986 
[57] L. A. Hageman and D. M. Young, Applied lterative Methods. New York, NY: Academic, 1981.

[58] M. Haghoo and W. Proskurowski, "Parallel efficiency of a domain decomposition method," in Domain Decomposition Methods, T. F. Chan et al., Eds. Philadelphia, PA: SIAM, 1989, pp. 269-281.

[59] R. W. Hockney, "A fast direct solution of Poisson's equation using Fourier analysis," J. Assoc. Comp. Mach., vol. 12, pp. 95-113, 1965.

[60] -, "The potential calculation and some applications," in Methods of Computational Physics, vol. 9, B. Adler, S. Fernbach, and M. Rotenberg, Eds., New York, NY: Academic, 1969, pp. 136-211.

[61] K. Hwang, "Advanced parallel processing with supercomputer architectures," Proc. IEEE, vol. 75, pp. 1348-1379, 1987.

[62] K. Hwang and F. A. Briggs, Computer Architecture and Parallel Processing. New York, NY: McGraw-Hill, 1984.

[63] D. C. Jespersen, "Multigrid methods for partial differential equations," in Studies in Numerical Analysis, G. H. Golub, Ed. Providence, RI: Math. Assoc. Amer., 1984.

[64] O. G. Johnson, C. A. Micchelli, and G. Paul, "Polynomial preconditioning for conjugate gradient calculations," SIAM J. Numer. Anal., vol. 20, pp. 362-376, 1983.

[65] L. V. Kantorovich and V. I. Krylov, Approximate Methods of Higher Analysis. New York, NY: Interscience Publishers, 1964.

[66] R. Kettler, "Analysis and comparison of relaxation schemes in robust multigrid and preconditioned conjugate gradient methods," in Multigrid Methods, W. Hackbush U. Trottenberg, Eds. New York, NY: Springer-Verlag, 1982, pp. 502-534.

[67] R. Kettler and J. A. Meijerink, "A multigrid method and a combined multigrid-conjugate gradient method for elliptic problems with strongly discontinuous coefficients in general domain," Shell publication 604, KSEPL, Rijswijk, The Netherlands, 1981.

[68] D. E. Keyes and W. D. Gropp, "A comparison of domain decomposition techniques for elliptic partial differential equations and their parallel implementation," SIAM J. SCi. Stat. Comput., vol. 8, pp. s166-s202, 1987.

[69] C.-C. J. Kuo, "Discretization and solution of elliptic PDEs: a transform domain approach," Ph.D. dissertation, Dept. of Electrical Engineering and Computer Science, Massachusetts Institute of Technology, Cambridge, MA, 1987.

[70] C.-C. J. Kuo and T. F. Chan, "Two color Fourier analysis of iterative algorithms for elliptic problems with red/black ordering," SIAM J. Sci. Stat. Comput., vol. 11, pp. 767-793, 1990.

[71] C.-C. J. Kuo, T. F. Chan, and C. Tong, "Multilevel filtering elliptic preconditioners," SIAM J. Matrix Anal. Appl., vol. 11, pp. 403-429, 1990.

[72] C.-C. J. Kuo and B. C. Levy, "Mode-dependent finite-difference discretization of linear homogeneous differential equations," SIAM J. Sci. Stat. Comput., vol. 9, pp. 992-1015, 1988.

[73] _- "A two-level four-color SOR method," SIAM J. Numer. Analy., vol. 26, pp. 129-151, 1989.

[74] _-, "Two-color Fourier analysis of the multigrid method with red/black Gauss-Seidel Smoothing," Applied Math. and Comput., vol. 29, pp. 69-87, 1989.

[75] C.-C. J. Kuo, B. C. Levy, and B. R. Musicus, "A local relaxation method for solving elliptic PDEs on mesh-connected arrays," SIAM J. Sci. Stat. Comput., vol. 8, pp. 550-573, Jul. 1987.

[76] Y.A. Kuznetsov, "Multigrid domain decomposition methods for elliptic problems," in Proc. VIIIth Internat. Conf. on Computational Methods for Applied Science and Eng., vol. 2, pp. 605-616, 1987.

[77] R. J. LeVeque and L. N. Trefethen, "Fourier analysis of the SOR iteration," IMA J. Numer. Anal., vol. 8, pp. 273-279, 1988.

[78] J. S. Lim, Two-dimensional Signal and Image Processing. Englewood Cliffs, NJ: Prentice-Hall, 1990.

[79] J. Mandel et al., Eds., Proceedings of the Fourth Copper
Mountain Conference on Multigrid Methods. Philadelphia, PA: SIAM, 1989.

[80] R. Manohar and J.W. Stephenson, "Optimal finite analytic methods" /. Heat Transfer, vol. 104, pp. 432-437, 1982.

[81] S. F. McCormick, Ed., Multigrid Methods. Philadelphia, PA: SIAM, 1987.

[82] J. A. Meijerink and H. A. van der Vorst, "An iterative solution method for linear systems of which the coefficient matrix is a symmetric M-matrix," Math. Comp., vol. 31, pp. $148-162,1977$.

[83] R. A. Nicolaides, "On the $l^{2}$ convergence of an algorithm for solving finite element equations," Math. Comp., vol. 31, pp. 892-906, 1977.

[84] M. E. G. Ong, "Hierarchical basis preconditioners for second order elliptic problems in three dimensions," Ph.D. dissertation, Dept. of Applied Mathematics, Univ. of Washington, Seattle, WA, 1989.

[85] A. V. Oppenheim and R. W. Schafer, Digital Signal Processing. Englewood Cliffs, NJ: Prentice-Hall, 1975.

[86] J. M. Ortega, Introduction to Parallel and Vector Solution of Linear Systems. New York, NY: Plenum, 1988.

[87] J. M. Ortega and R. G. Voigt, "Solution of partial differential equations on vector and parallel computers," SIAM Review, vol. 27 , pp. 149-240, 1985.

[88] M. H. Schultz, Elliptic Problem Solvers. New York, NY: Academic, 1981.

[89] H. A. Schwarz, Gesammelte Mathematische Adhandlungen, vol. 2, pp. 133-143, Berlin: Springer, 1890.

[90] G. Strang, Introduction to Applied Mathematics. Cambridge, MA: Wellesley-Cambridge Press, 1986.

[91] G. Strang and G. Fix, "A Fourier analysis of the finite element method," in Constructive Aspects of Functional Analysis. Rome, Italy: Edizioni Cremonese, 1973.

[92] K. Stuben and U. Trottenberg, "Multigrid methods: fundamental algorithms, model problem analysis, and applications," in Multigrid Methods, W. Hackbusch and U. Trottenberg, Eds. New York, NY: Springer-Verlag, 1982, pp. 1176.

[93] P.N. Swarztrauber, "The methods of cyclic reduction, Fourier analysis and the FACR algorithms for the discrete solu tion of Poisson's equation on a rectangle," SIAM Review, vol. 19, pp. 490-501, 1977.

[94] P. N. Swarztrauber, "Fast Poisson Solvers," in Studies in Numerical Analysis, G. H. Golub, Ed. Providence, RI: Math. Assoc. Amer., 1984, pp. 319-370.

[95] C. Tong, T. F. Chan, and C.-C. J. Kuo, "Multilevel filtering preconditioners: extensions to more general elliptic problems," Tech. Rep., Dept. of Mathematics, University of California, Los Angeles, May 1990.

[96] R. S. Varga, Matrix Iterative Analysis. Englewood Cliffs, N]: Prentice-Hall, 1962.

[97] P. Vassilevski, "Iterative methods for solving finite element elliptic equations based on the multilevel splitting of the matrix," Report 1989-09, Institute for Scient. Comput., Univ. of Wyoming, Laramie, 1989.

[98] O. Widlund, "Domain decomposition algorithms and the bicentennial of the French revolution," SIAM News, vol. 22 , no.4, July 1989.

[99] J. Xu, "Theory of multilevel methods," Ph.D. dissertation, Dept. of Mathematics, Cornell University, NY, 1989.

[100] D. M. Young, "Iterative methods for solving partial differential equations of elliptic type," Ph.D. dissertation, Harvard Univ., 1950.

[101] D. M. Young, "Iterative methods for solving partial differential equations of elliptic type," Trans. Amer. Math. Soc., vol. 76, pp. 92-111, 1954.

[102] D. M. Young, Iterative Solution of Large Linear Systems. New York, NY: Academic, Inc., 1971.

[103] D. M. Young, "A historical overview of iterative methods," Computer Physics Communications, vol. 35, pp. 1-17, 1989

[104] $H$. Yserentant, "On the multi-level splitting of finite element spaces," Numer. Math., vol. 49, pp. 379-412, 1986.

[105] H. Yserentant, "Hierarchical bases give conjugate gradient type methods a multigrid speed of convergence," Appl. Math. and Comp., vol. 19, pp. 347-358, 1986. 


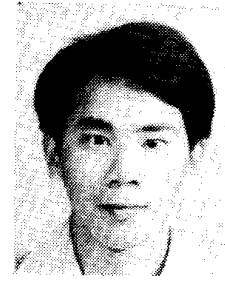

C.-C. Jay Kuo (Member, IEEE) was born in Hsinchu, Taiwan, in 1957. He received the B.S. degree from the National Taiwan University, Taipei, Taiwan, in 1980 and the M.S.E.E. and Ph.D. degrees from the Massachusetts Institute of Technology, Cambridge, in 1985 and 1987 , respectively, all in electrical engineering.

From October 1987 to December 1988 , he was Computational and Applied Mathematics (CAM) Research Assistant Professor in the Department of Mathematics at the University of California at Los Angeles. Since January 1989, he has been with the Department of Electrical Engineering-Systems at the University of Southern California, where he is currently Assistant Professor of Electrical Engineering. His research interests are in the areas of numerical analysis, digital signal processing and parallel computation.

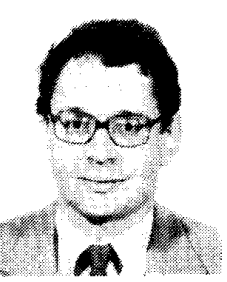

Bernard C. Levy (Senior Member, IEEE) was born in Princeton, NJ, on July 31, 1951. He received the diploma of Ingénieur Civil des Mines fromthe Ecole Nationale Supérieure des Mines in Paris, France, and the Ph.D. in electrical engineering from Stanford University, Stanford, CA.

While at Stanford University, he held an INRIA Fellowship, and worked also as Teaching Assistant, Research Assistant, and Instructor. From June 1979 to June 1987, he was Assistant, and then Associate Professor in the Department of Electrical Engineering and Computer Science at MIT. Since July 1987 , he has been with the University of California at Davis, where he is currently Professor of Electrical Engineering. His research interests are in the areas of multidimensional and statistical signal processing, inverse problems, estimation, detection, and scientific computing. 